\title{
WestVirginiaUniversity
}

THE RESEARCH REPOSITORY @ WVU

Graduate Theses, Dissertations, and Problem Reports

2017

\section{Optimization of Mineral Processing Circuit Design under Uncertainty}

Seyed Hassan Amini

Follow this and additional works at: https://researchrepository.wvu.edu/etd

\section{Recommended Citation}

Amini, Seyed Hassan, "Optimization of Mineral Processing Circuit Design under Uncertainty" (2017). Graduate Theses, Dissertations, and Problem Reports. 5097.

https://researchrepository.wvu.edu/etd/5097

This Dissertation is protected by copyright and/or related rights. It has been brought to you by the The Research Repository @ WVU with permission from the rights-holder(s). You are free to use this Dissertation in any way that is permitted by the copyright and related rights legislation that applies to your use. For other uses you must obtain permission from the rights-holder(s) directly, unless additional rights are indicated by a Creative Commons license in the record and/ or on the work itself. This Dissertation has been accepted for inclusion in WVU Graduate Theses, Dissertations, and Problem Reports collection by an authorized administrator of The Research Repository @ WVU.

For more information, please contact researchrepository@mail.wvu.edu. 


\title{
Optimization of Mineral Processing Circuit Design Under Uncertainty
}

\author{
Seyed Hassan Amini
}

\author{
Dissertation submitted \\ to the Benjamin M. Statler College of Engineering and Mineral Resources \\ at West Virginia University \\ in partial fulfillment of the requirements for the degree of \\ Doctor of Philosophy in \\ Mining Engineering
}

\author{
Aaron Noble, PhD., Chair \\ John A. Herbst, Ph.D. \\ Felicia F. Peng, Ph.D. \\ Mark F. Sindelar, Ph.D. \\ Rashphal Ahluwalia, Ph.D. \\ Department of Mining Engineering
}

Morgantown, West Virginia
2017

Keywords: mineral processing circuit design; uncertainty; linear circuit analysis; law of propagation of error; Taguchi's method; stochastic optimization

Copyright 2017 Seyed Hassan Amini 


\title{
ABSTRACT \\ Optimization of Mineral Processing Circuit Design Under Uncertainty
}

\author{
Seyed Hassan Amini
}

The estimation and analysis of uncertainty propagation in mineral processing separation circuits is an essential and significant, but challenging, aspect of a comprehensive optimal circuit design procedure. Owing to the sophisticated modeling requirements, many of the current circuit optimization tools rely on deterministic models, despite the ubiquity of uncertainty in the techno-economic input parameters (e.g., mineral price, plant feed grade, and process kinetic coefficients). While individual sources of uncertainty are substantial, the circuit designer must also estimate the compounded uncertainty imputed by the actual circuit design and identify which units are most influential in this uncertainty propagation. Additionally, despite the uncertainty in the input factors, the designer must identify precise specifications for the number and size of individual separation units with the objective of optimizing technical and economic performance measures. These factors must be considered very early in the design process due to the rigidity of the final flowsheet and early constraints of product specifications imposed by sales contracts. This dissertation seeks to resolve these issues by providing a suite of novel techniques that augment the state-of-the-art process models currently used by circuit designers. First, the linear circuit analysis approach and the law of propagation of error are combined to effectively analyze and evaluate circuit uncertainty propagation in the early design stages. Subsequently, the capability of this novel methodology is demonstrated to accurately recognize the most influential factors in the uncertainty contribution. Parallel to this part of the study, Taguchi's method is employed to evaluate the level of compounded circuit uncertainty while using fewer function evaluations compared to the common stochastic techniques, such as Monte Carlo simulation. Then, a systematic separation experimental study is performed using an electrostatic separator to validate the fundamental conclusions derived from the proposed methodologies. Finally, a comprehensive circuit optimization technique, based on the sample average approximation approach, is applied to determine the most profitable separation circuit configuration under uncertainty. Given the large flow volumes, high capital costs, and relative rigidity of the final flowsheet, findings of the current study will guarantee that a suitable separation circuit is selected relatively early in the design process 


\section{Acknowledgments}

The preparation of this dissertation has been an immensely rewarding undertaking. I would like to first thank the Lord for the many blessings I have experienced.

I would like to express my deepest appreciation to my Ph.D. advisor and my committee chair Dr. Aaron Noble, who has given me a vast degree of encouragement throughout my Ph.D. studies at West Virginia University. He continually and convincingly conveyed a spirit of adventure regarding research, and excitement regarding teaching. Without his guidance and persistent help, this dissertation would not have been possible.

I would like to thank my committee members, Dr. John Herbst, Dr. Felicia Peng, Dr. Mark Sindelar, and Dr. Rashphal Ahluwallia for their valuable time, helpful suggestions, and great support on my dissertation.

Many thanks are given to my friends, Mehrdad Shahabi, Xue Zhang, Scott Saber, Chris Vass, Amir Tafreshian, Armin Niaki, Kara Savage, Marcell Silveira, and all my fellow graduate students and colleagues for their technical supports and brainstorm communications, which helped me through the rough times. 


\section{Table of Contents}

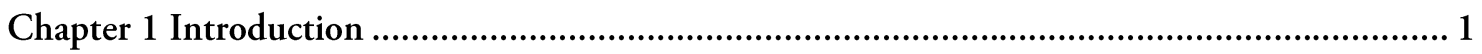

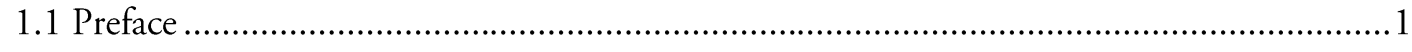

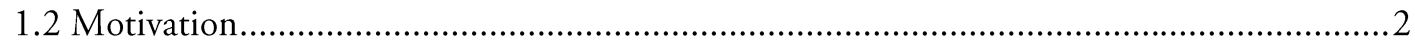

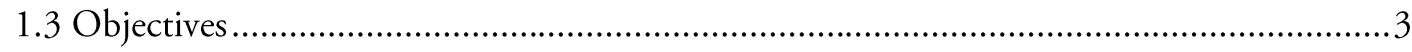

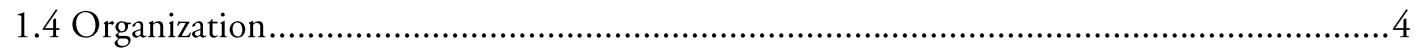

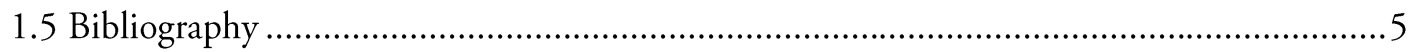

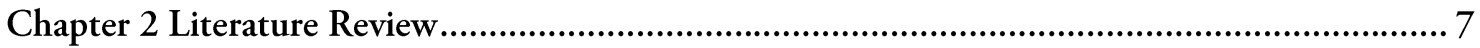

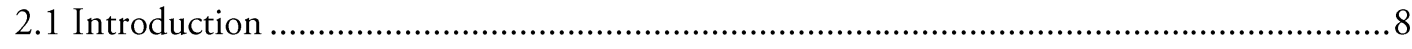

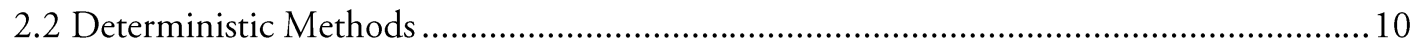

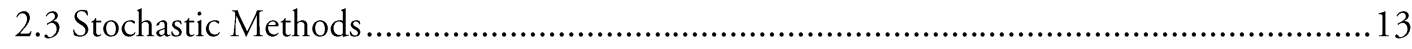

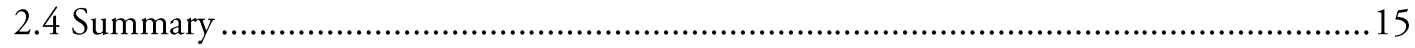

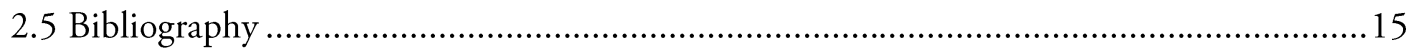

Chapter 3 Application of Linear Circuit Analysis in Evaluation of Mineral Processing Circuit

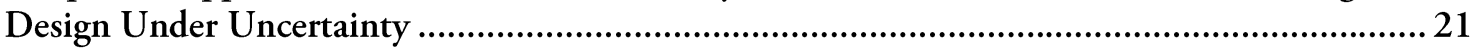

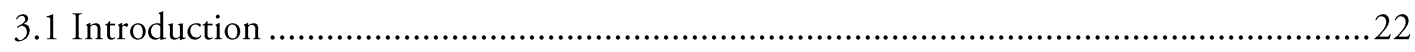

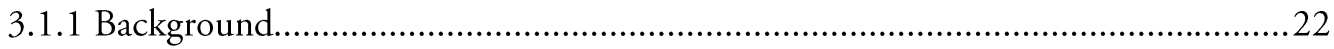

3.1.2 Uncertainty Consideration in Circuit Design .................................................24

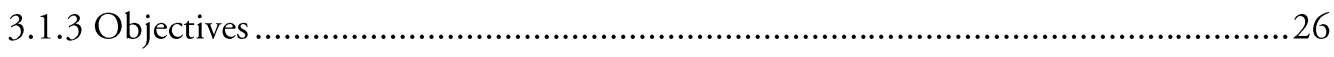

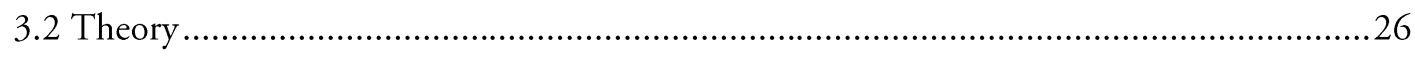

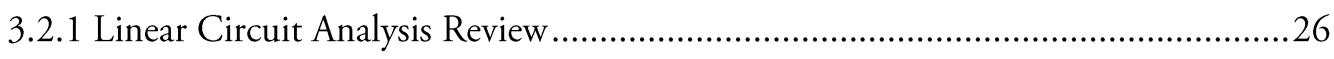

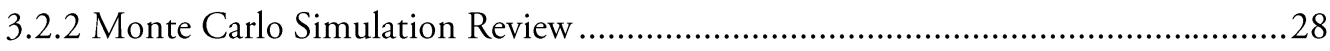

3.2.3 Derivation of an Uncertainty Evaluation Method Based on Linear Circuit

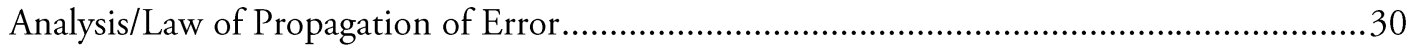

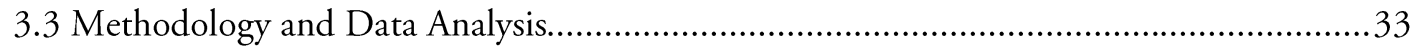

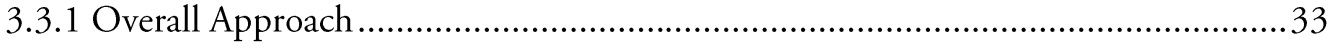

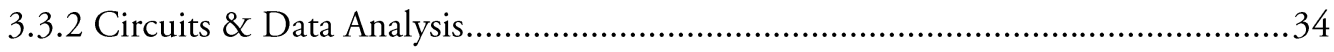

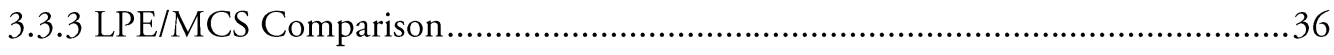

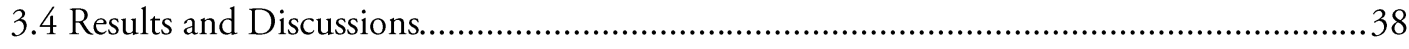

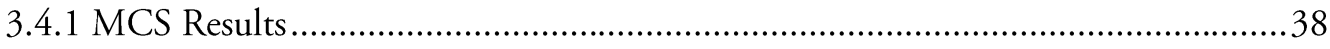




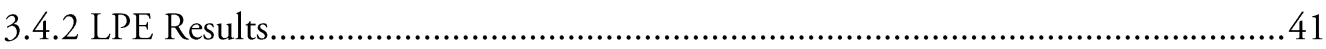

3.4.3 Discussion and Analysis of Input Parameter Variance............................................43

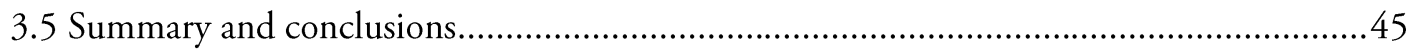

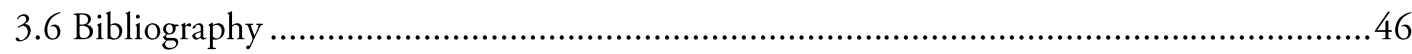

Chapter 4 Application of Linear Circuit Analysis and Functional Unit Evaluation to the Sensitivity

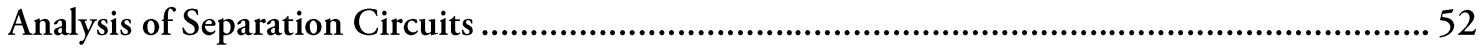

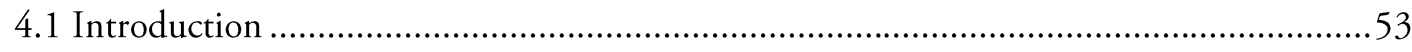

4.1.1 Background.............................................................................................

4.1.2 Sensitivity analysis for separation circuits.........................................................54

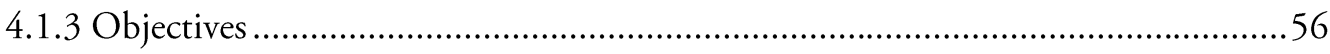

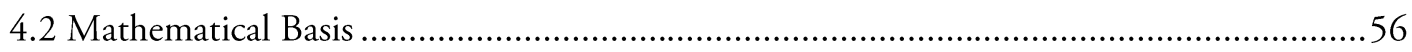

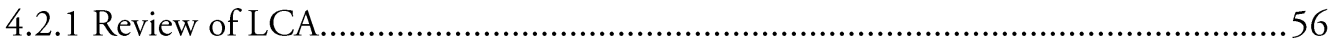

4.2.2 Review of LPE-LCA method...........................................................................58

4.2.3 Review of process functional units..................................................................6

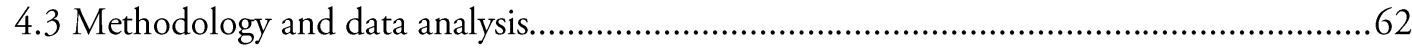

4.3.1 Sensitivity analysis using principles of functional units.......................................62

4.3.2 Sensitivity analysis using LPE-LCA approach ....................................................64

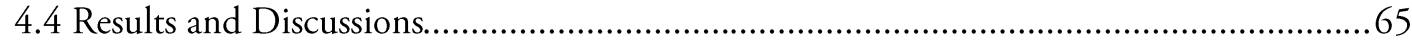

4.4.1 Sensitivity analysis using principles of functional units.........................................65

4.4.2 Results of global sensitivity analysis ..................................................................

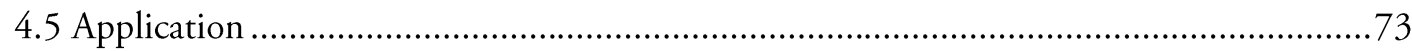

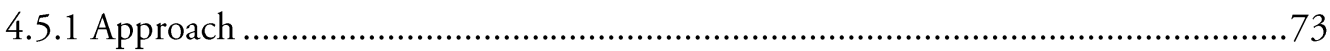

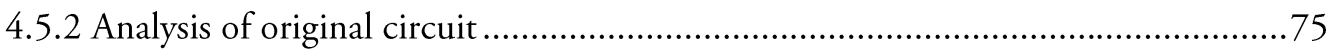

4.5.3 Analysis of alternative circuits........................................................................

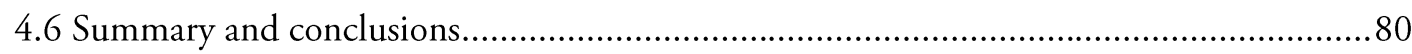

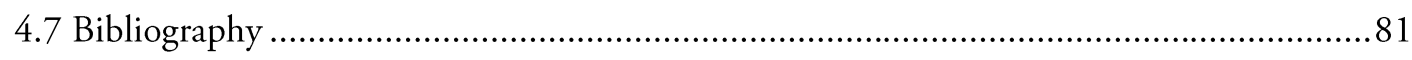

Chapter 5 Experimental Validation of Circuit Uncertainty Quantification Methodology ..........84

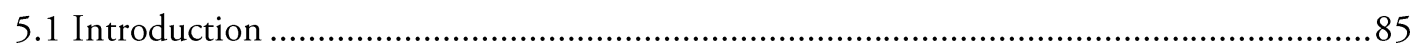

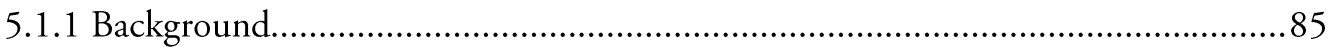

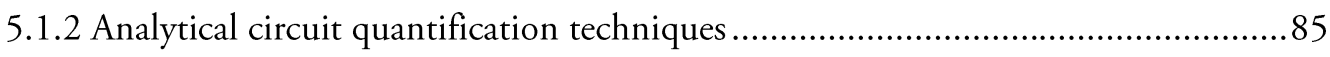

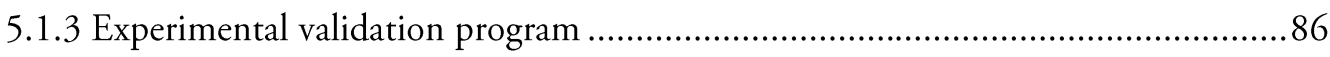




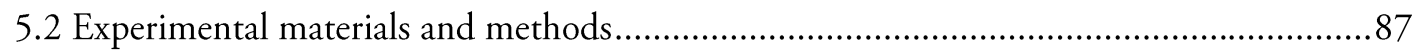

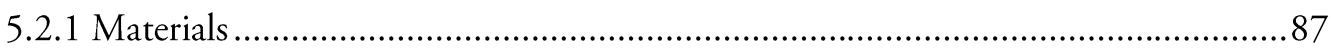

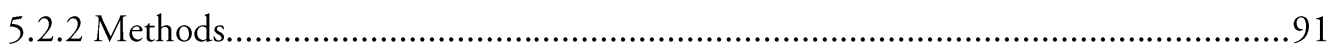

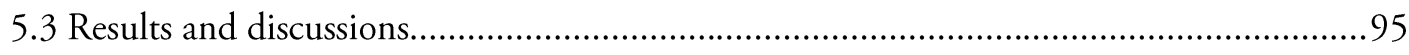

5.3.1 Scavenging capacity addition......................................................................... 96

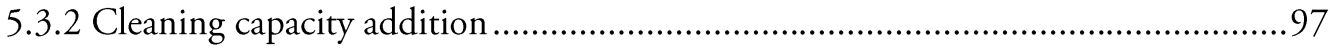

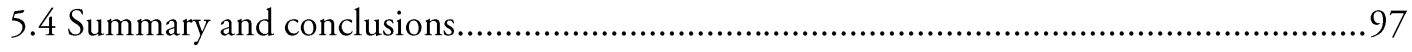

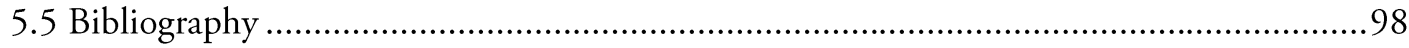

Chapter 6 Application of Taguchi's Method to Uncertainty Assessment for Mineral Processing

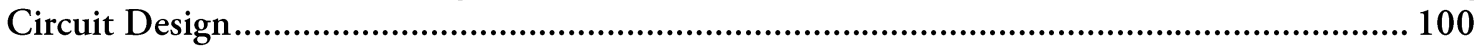

6.1 Introduction and method description.......................................................................

6.1.1 Uncertainty in Mineral Processing Circuit Design..............................................101

6.1.2 Monte Carlo Simulation .................................................................................. 101

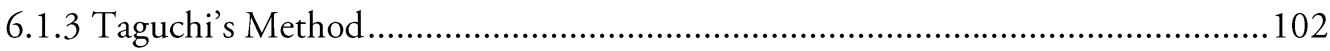

6.2 Application Example in Flotation Scale-Up............................................................... 104

6.3 Application Example in Separation Circuit Design ........................................................107

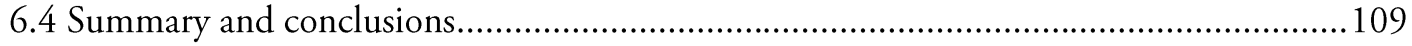

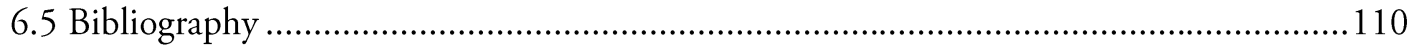

Chapter 7 A Techno-Economic Strategy for the Optimal Design of Cell-Based Flotation Circuits

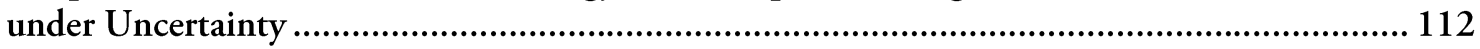

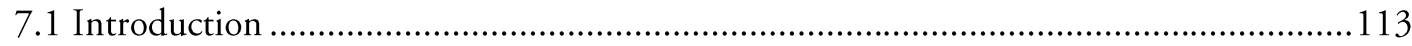

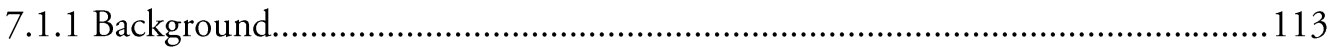

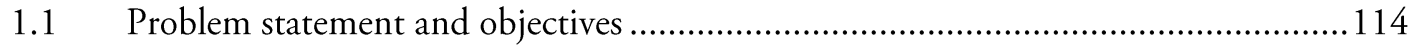

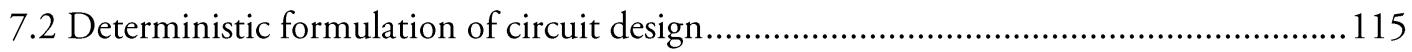

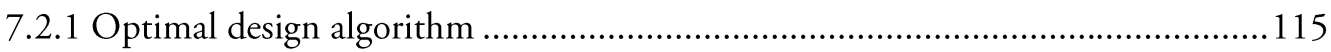

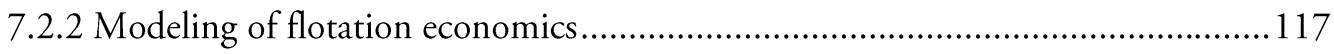

7.2.3 Modeling of flotation metallurgy .................................................................. 118

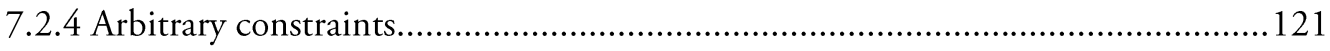

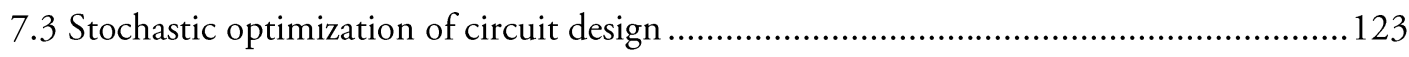

7.3.1 Sensitivity analysis of uncertain parameters …............................................... 124

7.3.2 Sample average approximation approach ..................................................... 124 


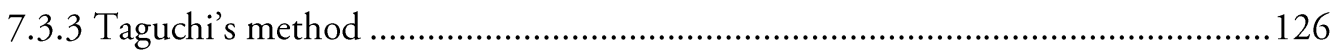

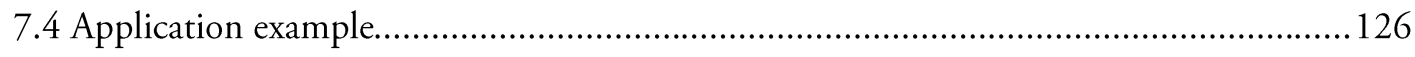

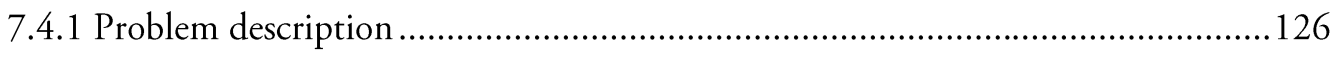

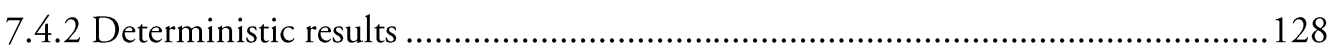

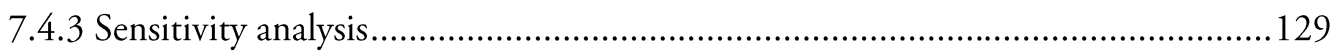

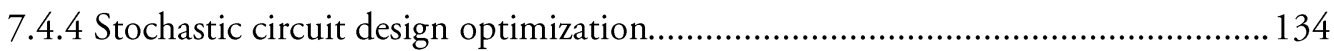

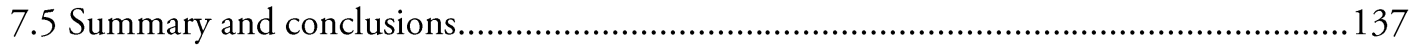

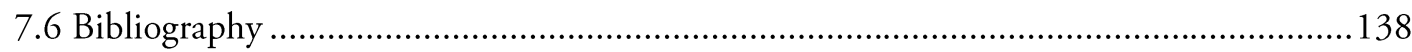

Chapter 8 Conclusions and Recommendations.................................................................... 141 


\section{Chapter 1}

\section{Introduction}

\subsection{Preface}

Mineral processing, also known as mineral/ore beneficiation, follows mining operations and prepares the final ore product for downstream users, including smelters, utilities, and other end-users. The main objective of the mineral processing operation is the separation of valuable (ore minerals) and waste components (gangue minerals) within dilute run-of-mine materials. Given the relatively low quality of run-of-mine materials, mineral processing is often an essential step for improving the marketable value of the raw materials. The profitability of mineral processing operation depends heavily on the compositional purity (i.e., grade) and production rate (i.e., recovery) of the final product. Consequently, an effective processing operation must maintain both ore recovery and product grade at economically-favorable levels (Fuerstenau and Han 2003; Wills 2011; Noble 2013).

In most cases, single separation units are technically and economically deficient in meeting final product specifications. Additionally, these single unit inefficiencies also lead to product variable and prediction uncertainty, which is then compounded by varying feed grades and slow lagging process control (Amini and Noble 2017). As a result, mineral processing operations often employ complex multi-stage circuit designs to mitigate these issues. The appropriate arrangement of separation units within a circuit configuration has the inherent potential to enhance the single-stage separation performance; however, poor circuit design degrades the performance below that of a single-unit case. Therefore, the optimal design of the separation circuit configuration is a complex and open-ended engineering problem that requires a comprehensive and deliberate approach (Noble 2013; Amini and Noble 2017). 
Unfortunately, the nature of the mining and mineral processing industry introduces considerable uncertainty into the separation circuit design procedure. Despite typical industrial processes that are largely impacted by internal process uncertainties, external sources of uncertainty, which namely include mineral price and run-of-mine characteristics, may dramatically affect the performance of mineral processing operation. The level of internal process uncertainties may be reduced via improvement in the control system. Nevertheless, the resultants external uncertainties from dynamic market factors and heterogeneity of geologic materials may not be mitigated (Simonsen and Perry 1999; Bárdossy and Fodor 2001; Der Kiureghian and Ditlevsen 2009; Caers 2011; Lisitsin et al. 2014; Jamett et al. 2015). Therefore, analysis of uncertainty propagation in separation circuits and consideration of these uncertainties in the design process is an essential aspect of an optimal circuit design procedure. Neglecting the significance of these uncertainties may result in failing to achieve an optimal, or even sub-optimal, circuit solutions.

This work is largely concerned with the analysis, evaluation, and optimization of mineral processing circuit designs under uncertainty. Given the unique characterizations of mineral ores in distinct locations, obviously, no single circuit is universally suitable in all instances. As such, several analytical and optimization tools are introduced to ease the circuit design decisions regardless of site, ore, and time conditions.

\subsection{Motivation}

Estimation and analysis of uncertainty propagation in mineral processing separation circuits is an essential and significant, but currently difficult, aspect of an optimal circuit design procedure. Most of the current circuit design methodologies have been constructed, implemented, and validated using a deterministic modeling approach. Nevertheless, numerous studies have shown that the inherent uncertainty induced by feed grade, operational conditions, and economic factors can be a significant parameter in many mineral processing applications.

While individual sources of uncertainty are substantial, circuit designers must also estimate the compounded uncertainty imputed by the actual circuit design and identify which units are most influential in this uncertainty propagation. Additionally, despite the 
uncertain operational and economic factors, they must precisely determine the number and size of individual separation units with the objective of optimizing technical and economic performance measures. These factors must be considered very early in the design process due to the rigidity of the final flowsheet and early constraints of product specifications imposed by sales contracts.

Thus, there is a critical need to develop an effective uncertainty quantification methodology and circuit design optimization strategy that can be implemented even with limited input data. In the absence of such methods, circuit designers may fail to achieve an optimal circuit solution that accurately reflects the stochastic influences that will compound as plants treat more sophisticated ore resources with diminishing feed grades.

\subsection{Objectives}

The singular goal of this research is to develop and validate novel and practical methodologies to systematically evaluate uncertainty propagation in mineral processing circuits and then optimize separation circuit configurations under uncertainty. These innovative approaches should streamline the circuit design procedure employing effective analytical methodologies to the fundamental circuit design strategies. The resultant methods provide invaluable tools for circuit designers who are working with limited information in the early phase of the design stage.

In summary, the itemized objectives of this study are to:

- Conduct a critical review of the recent advancements in separation circuit design process under uncertainty.

- Develop an analytical strategy to determine uncertainty propagation in mineral processing circuits.

- Assess the capability of the proposed analytical approach to explain the fundamental causes of circuit uncertainty propagation and predict the direction and degree of compounded uncertainty. 
- Develop a tool to identify influential parameters on separation performance uncertainty and evaluate the effectiveness of this methodology using various industrial case studies.

- Experimentally validate the developed circuit design techniques.

- Develop an innovative stochastic optimization methodology to determine the detailed design specifications of flotation stages with the objective of optimizing techno-economic performance measures.

\subsection{Organization}

The body of this dissertation is organized into eight chapters, with the primary works presented individually as standalone papers describing a separate phase or objective of the work. This approach differs from the traditional dissertation format in which each chapter is constructed based on the discussions and outcomes of the previous chapter/s. These primary phases constitute the six informative chapters, while an introductory and a concluding chapter complete the dissertation. References are listed individually for each chapter.

Chapter 1 summarizes the motivation for the current work, key objectives, and an overview of the work completed as a part of this study.

Chapter 2 provides a review of the state-of-the-art in the process of analysis, evaluation, and optimization of separation circuit design under uncertainty, with emphasis on the stochastic optimization methodologies.

Chapter 3 describes the development of an analytical technique for uncertainty quantification in mineral processing circuit configurations. The principles of linear circuit analysis approach, law of propagation of uncertainty, and Monte Carlo simulation are described. The utilization of this methodology is demonstrated for several generic separation circuit designs.

Chapter 4 presents the implementation of the proposed methodology, when combined with the principles of functional unit concept, as a global sensitivity analysis tool. This 
chapter provides several industrial case studies to effectively indicate the application of this technique in the circuit analysis procedure.

Chapter 5 validates the proposed circuit analysis and evaluation methodologies through the use of a systematic electrostatic separation tests.

Chapter 6 introduces the use of Taguchi's method, as an experimental design technique, in the process of analyzing mineral processing circuits under uncertainty.

Chapter 7 describes an innovative techno-economic strategy for the optimal design of cell-based flotation circuits under uncertainty. Sample Average Approximation approach and Taguchi's method are implemented to address the stochasticity of feed grade and kinetic coefficients in the flotation design procedure.

Chapter 8 summarizes the key findings and contributions of this work, including novel circuit design and optimization strategies for the cases of uncertainty, and introduces opportunities for further research and development.

\subsection{Bibliography}

Amini SH, Noble A. Application of linear circuit analysis in evaluation of mineral processing circuit design under uncertainty. Minerals Engineering. Elsevier; 2017;102:18-29.

Bárdossy G, Fodor J. Traditional and new ways to handle uncertainty in geology. Natural Resources Research. Springer; 2001;10(3):179-87.

Caers J. Modeling uncertainty in earth sciences. Wiley, Chichester; 2011. p. 246.

Fuerstenau MC, Han KN. Principles of mineral processing. SME; 2003.

Jamett N, Cisternas LA, Vielma JP. Solution strategies to the stochastic design of mineral flotation plants. Chemical Engineering Science. Elsevier; 2015;134:850-60. 
Der Kiureghian A, Ditlevsen O. Aleatory or epistemic? Does it matter? Structural Safety. Elsevier; 2009;31(2):105-12.

Lisitsin VA, Porwal A, McCuaig TC. Probabilistic fuzzy logic modeling: quantifying uncertainty of mineral prospectivity models using Monte Carlo simulations. Mathematical Geosciences. Springer; 2014;46(6):747-69.

Noble CA. Analytical and Numerical Techniques for the Optimal Design of Mineral Separation Circuits. Virginia Tech; 2013;

Simonsen H, Perry J. Risk identification, assessment and management in the mining and metallurgical industries. Journal-South African Institute of Mining and Metallurgy. South African Institute of Mining and Metallurgy; 1999;99(6):321-8.

Wills BA. Wills' mineral processing technology: an introduction to the practical aspects of ore treatment and mineral recovery. Butterworth-Heinemann; 2011. 


\title{
Chapter 2
}

\section{Literature Review}

\author{
(ABSTRACT)
}

Finding the optimal design of a mineral processing circuit is a complicated and open-ended design process. Over the last 40 years, numerous methodologies, including heuristic, analytical, and numerical optimization techniques, were utilized to select a suitable circuit configuration on the basis of one or more techno-economic performance indicators. Despite the utility of these predictive tools, they often only consider static input values and do not consider the uncertainty inherent to the input factors such as predicted unit recovery and feed grade. To address these concerns, recently several studies have described several approaches to analyze and predict the role of uncertainty in the circuit design procedure. However, only a few studies have attempted to optimize the circuit design parameters considering the stochasticity of input parameters. This chapter reports the most important studies in this field while emphasizing on the linear circuit analysis approach and stochastic mineral processing circuit design strategies.

Keywords: mineral processing circuit design; deterministic; stochastic; uncertainty. 


\subsection{Introduction}

Mineral processing and other physical separation plants often employ complex multiunit circuits to overcome the inherent inefficiencies of single unit operations in meeting final product specifications imposed by primary contracts. However, the optimal design of these circuits is a complex and open-ended engineering problem that requires a comprehensive and deliberate approach. An effective circuit design strategy must adequately address following questions to maximize separation circuit performance while minimizing risk: (1) the selection of the appropriate separation processes; (2) the selection of the number and size of individual units; (3) the selection of the various operational parameters for each unit; and (4) the configuration of the flows between units. The collective decision on these four questions may introduce an infinite number of solutions, including a very large number of viable or suboptimal circuit design solutions. Even though the implementation of adequate technical and economic constraints on each of these items may reduce the number of solutions, many alternative circuit design solutions may be produced. As such, to assist circuit designers in this complicated decision-making procedure, researchers have attempted to establish standard design methodologies which involve various numerical and analytical techniques. These tools are typically guided by some optimization tool and a generic process model applicable for the given problem. Unfortunately, most of the available technical-, financial-, or techno-economic-based optimization strategies do not consider the stochasticity of the mineral processing operation. Neglecting the significance of internal and external sources of uncertainty may result in failing to achieve the optimal mineral processing circuit design solution.

Circuit design analysis and optimization strategies can be described on a continuum scale depicting the level of direct mathematical involvement, as first described by Noble (2013). Figure 2-1, as the modified version, depicts the more recent studies and categorizes the available strategies into deterministic and stochastic classes. As shown in this figure, on the lower portion of the scale are purely heuristic methods. These circuit analysis techniques utilize rules and guidelines which may or may not be based on sophisticated mathematical integration. Conversely, purely numerical optimization routines define the higher portion of the scale. These methods have incorporated various optimization algorithms, including linear 


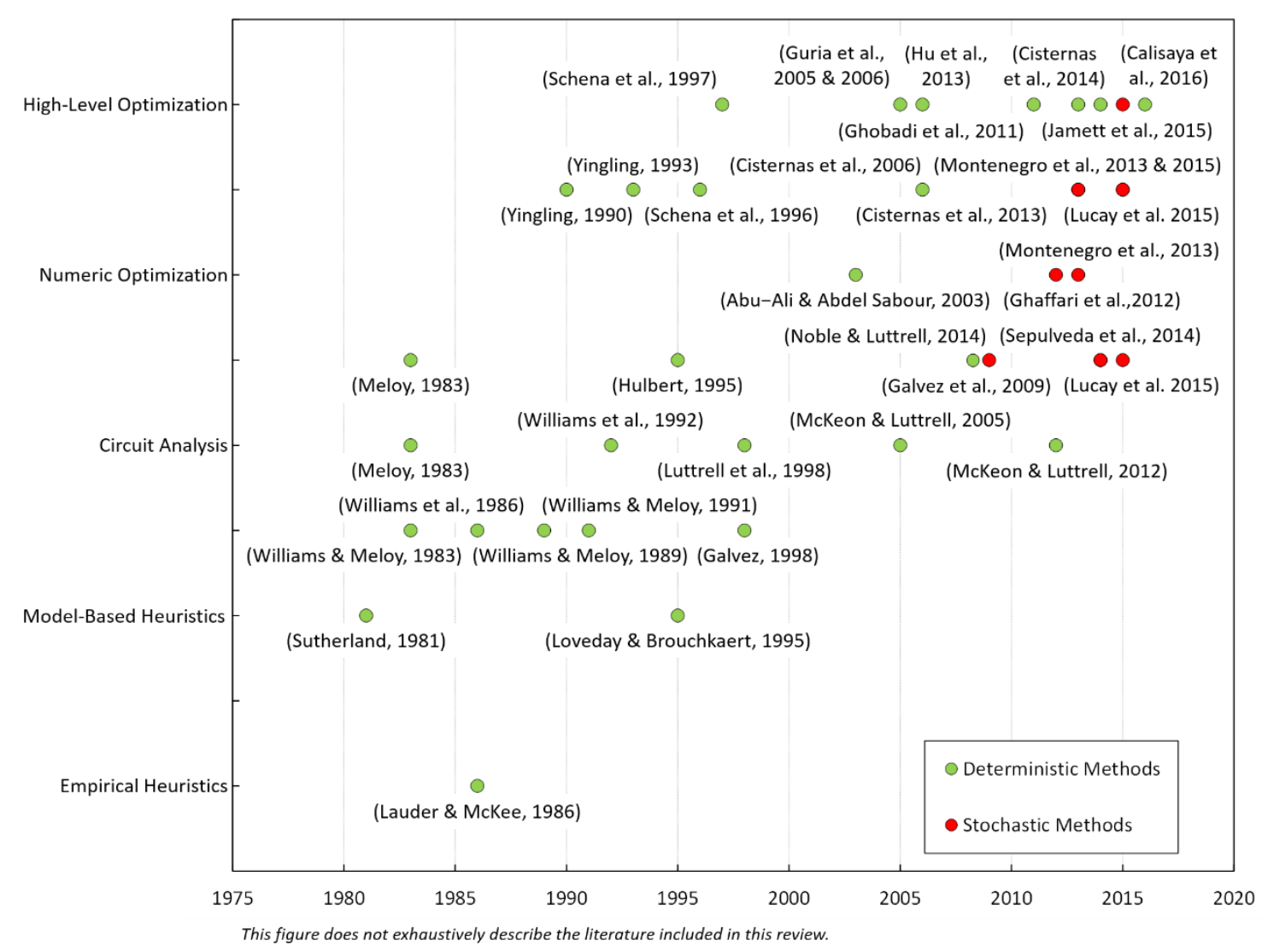

Figure 2-1: Survey of literature describing various methods of mineral processing circuit design and optimization.

programming, non-linear programming, gradient-based optimization, and genetic optimization. Contemporary trends are seemingly favoring high-tech numerical algorithms, based on either a deterministic or a stochastic strategy, to accommodate the nonlinear, discontinuous design parameters associated with separation circuits. Figure 2-1 also demonstrates that, during the last 40 years, most of the circuit design methods have incorporated deterministic modeling approaches. Nevertheless, several recent studies have indicated the significance of uncertainty consideration in mineral processing circuit design procedure. Consequently, several active research projects are developing strategies to find an optimal circuit solution given the inherent uncertainties in the separation circuits. This chapter will briefly review the state-of-the-art in these optimization strategies, emphasizing 
on linear circuit analysis and stochastic methodologies, while noting the merits and drawbacks of the different methodologies.

\subsection{Deterministic Methods}

Prior to the advent of process modeling and simulation tools, most circuit designs were determined by heuristic methodologies, established industry practices, and accepted "rulesof-thumb" (Lauder and McKee 1986; Wills 2011; Lucay et al. 2012). The heuristic approaches have been imposed design principles from either empirical observations or from process models. Over a decade, several researchers (Sutherland 1981; Williams and Meloy 1983, 1989, 1991; Williams et al. 1986; Loveday and Brouckaert 1995) provided various analytical and model-based tools to evaluate and improve separation circuit performance. Given the low level of complexity and lack of mathematical rigor, these strategies are easy to implement and, even today, are widely-accepted in the industry. However, since they rely on experiential rules, these classical methodologies may totally fail to achieve an optimal solution and may provide conflicting solutions (Mendez et al. 2009; Lucay et al. 2012).

Over the last 30 years, these heuristic approaches have been slowly displaced by different analytical methodologies and optimization techniques. In 1983, Meloy introduced linear circuit analysis (LCA) approach as an analytical tool to evaluate and rank separation circuit designs through simplified algebraic formulations (Meloy 1983). The method is particularly useful in the preliminary design stages, as it does not rely on a strict process model and thus requires limited a priori information on the equipment specifications and feed conditions. The concept of LCA was first derived to provide a method of optimizing multiunit separation circuit configurations. The original paper eventually developed into a series of publications examining various aspects and applications of the methodology. The LCA methodology is formulated based on separation probabilities. As a result, a separation unit's yield of a particular particle type is defined by a transfer function (or probability, $P$ ). The mass of material in the concentrate stream is simply the product of the yield and the feed mass $(P F)$, while the transfer function to the tailings stream constitutes the remaining material $(1-P)$. By extending this algebra over many units, the recovery for the entire circuit may be analytically defined in terms of each unit's recovery. Figure 2-2 shows examples of 


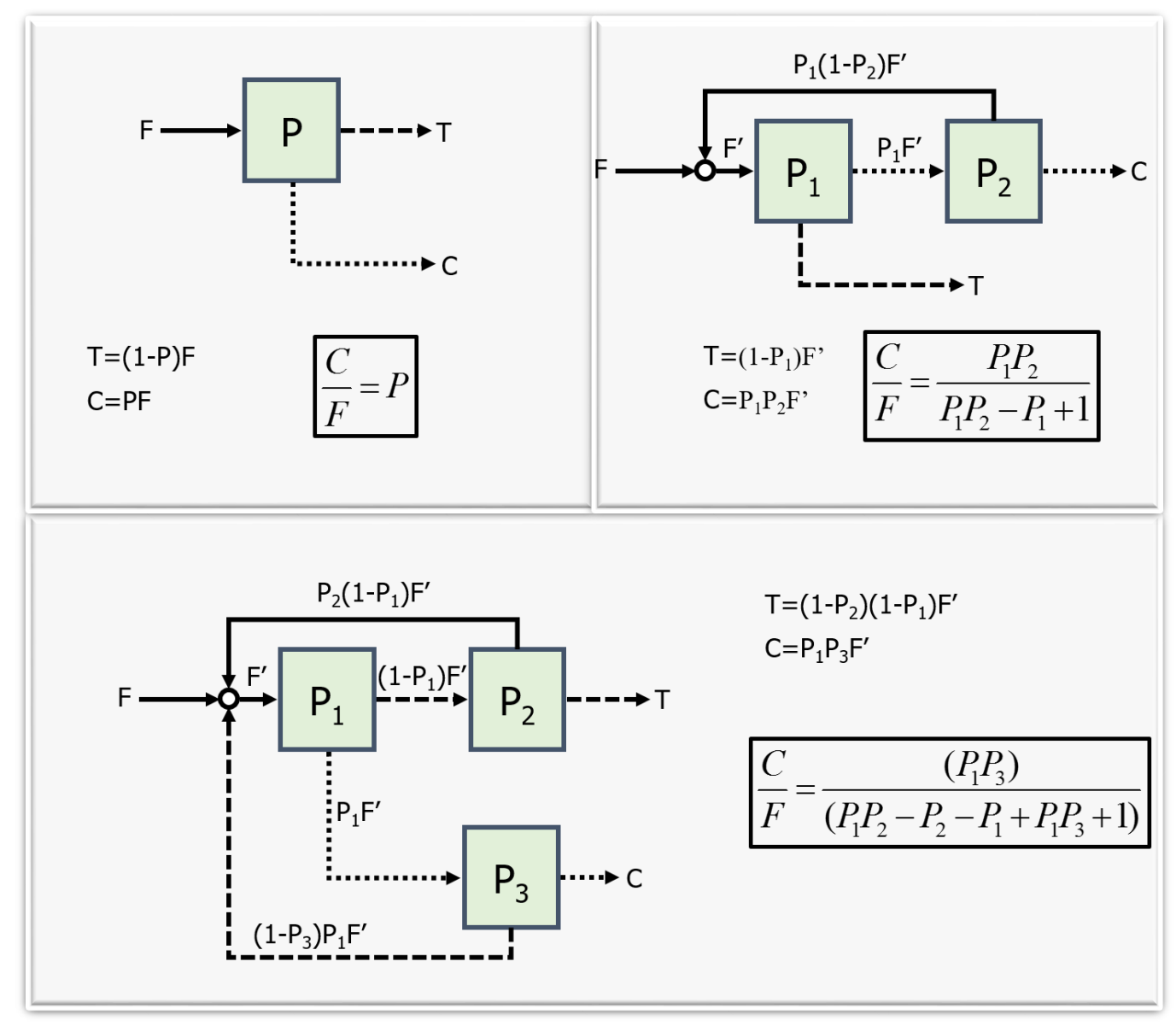

Figure 2-2: Examples of linear circuit analysis applied to several simple configurations.

this algebra applied to common circuit configurations. The power of all LCA applications is then derived from the analytical solution.

LCA is fundamentally restricted by the assumption of process linearity, which specifies that a unit's partition function is not influenced by particle-particle interactions. While this assumption is not wholly valid for processing plants, several industrial applications have verified linearity in some cases via experimental investigations (Harris and Cuadros-Paz 1978; Williams and Meloy 1983; Williams et al. 1986); and the practical utility of LCA has been repeatedly demonstrated in both greenfield (Luttrell et al. 1998) and operating (McKeon and Luttrell 2005; McKeon et al. 2012) plant designs. Detailed descriptions and 
applications of these techniques have been thoroughly explained in the literature and include: original LCA derivation and separation sharpness (Meloy 1983); identification of the most influential separation units (Lucay et al. 2012); streamlined analytical solution derivation (Noble 2013; Noble and Luttrell 2014); and techno-economic separation efficiency and yield determination (Noble and Luttrell 2015). Altogether, these calculations bring considerable value to the LCA approach, since they allow relative comparisons solely from the stream interconnections. Since the technique does not require information on feed conditions or equipment, LCA provides means to determine the fundamental characteristics of stream interconnections that lead to improved performance.

Several authors over the last 25 years have used numerical techniques in the circuit design problem, chiefly phenomenological process modeling and simulation (Lynch et al. 1981; King 2012). With the advent and development of these techniques, circuit selection has favored a more rigorous approach since these tools provide a means to assess circuits on the basis of one or more techno-economic performance indicators. To hasten these approaches, numerous researchers have identified optimization approaches that mitigate the need for blind iterative simulation, and a comprehensive review of these strategies has been presented by Mendez et al. (2009) and Yingling (1993). Most of these circuit optimization approaches begin by generating all combinations of possible circuit designs, commonly referenced as a superstructure. A search strategy is then used to reduce the number of alternative circuits in the superstructure, and process models can finally be applied to the unit operations to optimize technical-economic objective functions. An optimal (or pseudooptimal) solution may be obtained by utilizing one of several search algorithms, including: genetic search algorithms (Guria et al. 2005a, 2005b, 2006; Ghobadi et al. 2011; Hu et al. 2013), mixed integer programming (Cisternas et al. 2006; Calisaya et al. 2016), integer programming, and other methods (Yingling 1990, 1993; Schena et al. 1996, 1997). Despite the merits of these works, contemporary industry practice still favors classical heuristic solutions since optimally-derived circuits occasionally produce impractical results, and the optimization approaches are considerably complex. The search algorithms are strongly dependent on the input data and the accuracy of the process models. For example, Cisternas et al. (2014) has shown that the optimal circuit selection is highly dependent on the mineral market price and the specific objective function used in the analysis. 


\subsection{Stochastic Methods}

As shown in Figure 2-1, most of the preceding circuit design methods have been developed using a deterministic modeling approach. Nevertheless, several researchers have shown that uncertainty can be a significant factor in many mining and mineral processing applications. Kraslawski (1989) extensively reviewed various economic and technical uncertainties in chemical processing operations, including uncertainty in the final product price, feed conditions, and kinetic constants. From a mineral processing perspective, Ghaffari et al. (2012) systematically analyzed mineral assays and flow rates of several streams in a leadzinc flotation plant. Over the 3-month period of this investigation, the plant data clearly showed the variability of different factors, such as feed grade and flow rate as well as unit recovery. Moreover, the data addressed the dramatic impact that these uncertainties have on the separation performance of the flotation circuit. Other researchers have also evaluated the influence of parameter uncertainty on the variability of flow rates in flotation circuits (Xiao and Vien 2003) and the variance of SAG mill power draw (Karamoozian et al. 2008). The summative results from these studies show that process uncertainties must be incorporated in the circuit design procedure to achieve an optimal circuit configuration.

Uncertainty is a broad term and may be haphazardly applied in the context of mineral processing plant design. Generally, sources of uncertainty in physical operations may include measurement errors, systematic errors, natural variation, inherent randomness and subjective judgments (Morgan et al. 1992; Hoffman and Hammonds 1994; Regan et al. 2002). Simonsen and Perry (1999) proposed one method of classifying uncertainty in the mining operations by identifying the final parameter where the uncertainty is expressed. This classification identified various uncertainty classes including market price, ore reserve, mining costs, schedule duration, mining methods. Other researchers use a more mechanistic classification, which instead isolates the fundamental cause of the uncertainty as either epistemic (i.e. lack of knowledge) or stochastic/aleatory (i.e. inherent randomness that arises from natural heterogeneity) (Bárdossy and Fodor 2001; Der Kiureghian and Ditlevsen 2009; Caers 2011; Lisitsin et al. 2014; Jamett et al. 2015). Epistemic uncertainty may be reduced by further investigations; however, stochastic uncertainty may not be eliminated or even reduced in many cases. During the circuit design stage, the inherent variability of the final product price is determined by the commodity market and, similar to the feed and ore grade 
parameters, represents stochastic uncertainty. Alternatively, uncertainty in the separation unit recovery is an epistemic uncertainty and can be varied by using different design conditions and operational parameters (Jamet et al. 2015).

While individual causes of uncertainty are significant, the circuit designer must also consider the compounded uncertainty imputed by the actual circuit design and detect the most prominent parameters in the level of induced uncertainty and then merit further attention. Furthermore, circuit designers must determine the optimum number and size of different separation stages while considering the role of various uncertain techno-economic parameters. As such, over the last decade, few researchers have studied the impact of uncertain input data on the final separation performance for various circuit configurations (Gálvez 2009; Cisternas et al. 2015; Jamett et al. 2015; Montenegro et al. 2015). Most of these studies have utilized Monte Carlo Simulation approach, as a widely used simulation method to quantitatively evaluate uncertainty and variability in risk assessments by determining the distribution of expected outcomes from a standard deterministic process model. Subsequently, they have considered multiple uncertain input parameters to evaluate the influence that the input distribution type, uncertainty level, and circuit design have on the final output distribution.

Meanwhile, Lucay et al. (2012), Sepúlveda et al. (2013, 2014), and Lucay et al. (2015) evaluated the significance of each individual separation unit in determining the variability of global recovery using local and global sensitivity analysis methodologies. Given the analytical circuit transfer function, Lucay et al. (2012) used local sensitivity analysis techniques to examine each separation unit individually and the final results were compared to determine the most influential unit. Due to drawbacks of local sensitivity analysis approach, Sepúlveda et al. $(2013,2014)$ and Lucay et al. (2015) utilized various global sensitivity analysis strategies to simultaneously determine the role of each uncertain parameter in the circuit uncertainty propagation and then identify the most important uncertain factors.

Additionally, Jamett et al. (2015) applied stochastic optimization method in flotation circuit design problem using a simple flotation model to maximize profit while considering variable feed grade and uncertain metal price. The authors first introduced flotation circuit configurations based on generic representation of separation stages and then solved the 
stochastic problem, at nine distinct scenarios, with objective of maximizing profit considering different scenarios with various levels of uncertain input data. Consequently, they compared the final solution of the stochastic problem with deterministic approach and concluded that uncertainty consideration may offer better solution while resulting in achieving higher profit. Finally, the sensitivity analysis method was conducted to evaluate the effect of uncertainty in the circuit design process.

\subsection{Summary}

The literature briefly reviewed several methodologies for optimizing the separation circuit design and parameters in mineral processing industry. Before the original advent of simulation and modeling, most circuit configurations were designed from historic and legacy perspectives. Modeling and simulation led to more sophisticated and scientifically-based heuristics; but the final solutions are strongly dependent on the applicability of the initial assumptions and the robustness of the process model. Furthermore, majority of these simulation and optimization strategies require extensive amount of techno-economic information to find optimal or, in most cases, sub-optimal solutions. During this time, circuit analysis ascended as an analytical methodology which considered the fundamental capacity of the circuit itself, omitting the need for extensive operational-economic parameters, and allow circuit designers to understand the fundamental of unit interconnections. The ultimate adaptation to circuit analysis is realized in numeric circuit optimization. Recently, few studies have demonstrated the significance of operational-financial uncertainties in the circuit design problem and highlight the need for fundamental understanding on the mechanisms that cause uncertainty as well as the need for systematic analysis of separation circuit designs under uncertain conditions. However, finding an optimal separation circuit design, during early design stages, that maximizes techno-economic measures is still an open-ended engineering problem.

\subsection{Bibliography}

Bárdossy G, Fodor J. Traditional and new ways to handle uncertainty in geology. Natural Resources Research. Springer; 2001;10(3):179-87.

Caers J. Modeling uncertainty in earth sciences. Wiley, Chichester; 2011. p. 246. 
Calisaya DA, López-Valdivieso A, Marcos H, Gálvez EE, Cisternas LA. A strategy for the identification of optimal flotation circuits. Minerals Engineering. Elsevier; 2016;96:157-67.

Cisternas LA, Jamett N, Gálvez ED. Approximate recovery values for each stage are sufficient to select the concentration circuit structures. Minerals Engineering. Elsevier; 2015;83:175-84.

Cisternas LA, Lucay F, Gálvez ED. Effect of the objective function in the design of concentration plants. Minerals Engineering. Elsevier; 2014;63:16-24.

Cisternas LA, Méndez DA, Gálvez ED, Jorquera RE. A MILP model for design of flotation circuits with bank/column and regrind/no regrind selection. International Journal of Mineral Processing. Elsevier; 2006;79(4):253-63.

Gálvez EDSFDMMR. Sensitivity Assessment of Flotation Circuit to Uncertainty using Monte Carlo simulation. CRC Press. 2009;679-88.

Ghaffari A, Hayati M, Shekholeslami A. Probability and Sensitivity Analysis in Flotation Circuit of Bama Lead and Zinc Processing Plant Using Monte Carlo Simulation Method. Mineral Processing and Extractive Metallurgy Review. Taylor $1 \&$ Francis; 2012;33(6):416-26.

Ghobadi P, Yahyaei M, Banisi S. Optimization of the performance of flotation circuits using a genetic algorithm oriented by process-based rules. International Journal of Mineral Processing. Elsevier; 2011;98(3):174-81.

Guria C, Varma M, Mehrotra SP, Gupta SK. Simultaneous optimization of the performance of flotation circuits and their simplification using the jumping gene adaptations of genetic algorithm-II: More complex problems. International Journal of Mineral Processing. Elsevier; 2006;79(3):149-66.

Guria C, Verma M, Gupta SK, Mehrotra SP. Simultaneous optimization of the performance of flotation circuits and their simplification using the jumping gene adaptations of genetic algorithm. International Journal of Mineral Processing. Elsevier; 2005 a;77(3):165-85. 
Guria C, Verma M, Mehrotra SP, Gupta SK. Multi-objective optimal synthesis and design of froth flotation circuits for mineral processing, using the jumping gene adaptation of genetic algorithm. Industrial \& engineering chemistry research. ACS Publications; 2005 b;44(8):2621-33.

Harris C, Cuadros-Paz A. Species interaction in flotation: a laboratory-scale semi-batch study. International Journal of Mineral Processing. Elsevier; 1978;5(3):267-83.

Hoffman FO, Hammonds JS. Propagation of uncertainty in risk assessments: the need to distinguish between uncertainty due to lack of knowledge and uncertainty due to variability. Risk analysis. Wiley Online Library; 1994;14(5):707-12.

$\mathrm{Hu}$ W, Hadler K, Neethling S, Cilliers J. Determining flotation circuit layout using genetic algorithms with pulp and froth models. Chemical Engineering Science. Elsevier; 2013;102:32-41.

Jamett N, Cisternas LA, Vielma JP. Solution strategies to the stochastic design of mineral flotation plants. Chemical Engineering Science. Elsevier; 2015;134:850-60.

Karamoozian M, Shafaei S, Kakaie R, Noaparast M, Zeidabadi S, Ghasemi S. SAG mill power draw simulation using variance propagation approach. Iranian Journal of Science and Technology, Transaction B, Engineering. 2008;32(B6):619-29.

King RP. Modeling and simulation of mineral processing systems. Elsevier; 2012.

Der Kiureghian A, Ditlevsen O. Aleatory or epistemic? Does it matter? Structural Safety. Elsevier; 2009;31(2):105-12.

Kraslawski A. Review of applications of various types of uncertainty in chemical engineering. Chemical Engineering and Processing: Process Intensification. Elsevier; 1989;26(3):185-91.

Lauder D, McKee D. The impact of circulating loads on flotation circuit performance. 13th CMMI Congress. 1986. p. 41-7.

Lisitsin VA, Porwal A, McCuaig TC. Probabilistic fuzzy logic modeling: quantifying uncertainty of mineral prospectivity models using Monte Carlo simulations. 
Mathematical Geosciences. Springer; 2014;46(6):747-69.

Loveday B, Brouckaert C. An analysis of flotation circuit design principles. The Chemical Engineering Journal and The Biochemical Engineering Journal. Elsevier; 1995;59(1):15-21.

Lucay F, Cisternas L, Gálvez E. Global sensitivity analysis for identifying critical process design decisions. Chemical Engineering Research and Design. Elsevier; 2015;

Lucay F, Mellado ME, Cisternas LA, Gálvez ED. Sensitivity analysis of separation circuits. International Journal of Mineral Processing. Elsevier; 2012;110:30-45.

Luttrell G, Kohmuench J, Stanley F, Trump G. Improving spiral performance using circuit analysis. Minerals \& metallurgical processing. Society for Mining, Metallurgy and Exploration; 1998;15(4):16-21.

Lynch A, Johnson N, Manlapig E, Thorne C. Mineral and coal flotation circuits: Their simulation and control. Elsevier, Amsterdam; 1981.

McKeon T, Luttrell G, others. Optimization of multistage circuits for gravity concentration of heavy mineral sands. Minerals \& Metallurgical Processing Journal. SME; 2012;29(1):1-5.

McKeon T, Luttrell GH. Application of linear circuit analysis in gravity separator circuit design. 2005.

Meloy T. Analysis and optimization of mineral processing and coal-cleaning circuits-circuit analysis. International Journal of Mineral Processing. Elsevier; 1983;10(1):61-80.

Mendez DA, Gálvez ED, Cisternas LA. State of the art in the conceptual design of flotation circuits. International Journal of Mineral Processing. Elsevier; 2009;90(1):1-15.

Montenegro MR, Gálvez ED, Cisternas LA. The effects of stage recovery uncertainty in the performance of concentration circuits. International Journal of Mineral Processing. Elsevier; 2015;143:12-7. 
Morgan MG, Henrion M, Small M. Uncertainty: a guide to dealing with uncertainty in quantitative risk and policy analysis. Cambridge University Press; 1992.

Noble A, Luttrell G. Value-based objective functions applied to circuit analysis. Minerals \& Metallurgical Processing. 2015;32(1).

Noble A, Luttrell GH. The matrix reduction algorithm for solving separation circuits. Minerals Engineering. Elsevier; 2014;64:97-108.

Noble CA. Analytical and Numerical Techniques for the Optimal Design of Mineral Separation Circuits. Virginia Tech; 2013;

Regan HM, Hope BK, Ferson S. Analysis and portrayal of uncertainty in a food-web exposure model. Human and Ecological Risk Assessment. Taylor \& Francis; 2002;8(7):1757-77.

Schena G, Villeneuve J, Noël Y. A method for a financially efficient design of cell-based flotation circuits. International journal of mineral processing. Elsevier; 1996;46(1):1-20.

Schena G, Zanin M, Chiarandini A. Procedures for the automatic design of flotation networks. International journal of mineral processing. Elsevier; 1997;52(2):137-60.

Sepúlveda FD, Cisternas LA, Gálvez ED. Global sensitivity analysis of a mineral processing flowsheet. In: Andrzej Kraslawski and Ilkka Turunen, editor. Proceedings of the 23rd European Symposium on Computer Aided Process Engineering-ESCAPE 23, Lappeenranta, Finland: Elsevier; 2013. p. 913-8.

Sepúlveda FD, Cisternas LA, Gálvez ED. The use of global sensitivity analysis for improving processes: Applications to mineral processing. Computers \& Chemical Engineering. Elsevier; 2014;66:221-32.

Simonsen H, Perry J. Risk identification, assessment and management in the mining and metallurgical industries. Journal-South African Institute of Mining and Metallurgy. South African Institute of Mining and Metallurgy; 1999;99(6):321-8.

Sutherland D. A study on the optimization of the arrangement of flotation circuits. International Journal of Mineral Processing. Elsevier; 1981;7(4):319-46. 
Williams M, Fuerstenau D, Meloy T. Circuit analysis-general product equations for multifeed, multistage circuits containing variable selectivity functions. International Journal of Mineral Processing. Elsevier; 1986;17(1):99-111.

Williams M, Meloy T. Dynamic model of flotation cell banks-circuit analysis. International Journal of Mineral Processing. Elsevier; 1983;10(2):141-60.

Williams M, Meloy T. On the definition and separation of fundamental process functions. International Journal of Mineral Processing. Elsevier; 1989;26(1):65-72.

Williams M, Meloy T. Feasible designs for separation networks: a selection technique. International journal of mineral processing. Elsevier; 1991;32(3):161-74.

Wills BA. Wills' mineral processing technology: an introduction to the practical aspects of ore treatment and mineral recovery. Butterworth-Heinemann; 2011.

Xiao Z, Vien A. Limitations of variance analysis using propagation of variance. Minerals engineering. Elsevier; 2003;16(5):455-62.

Yingling JC. Circuit analysis: optimizing mineral processing flowsheet layouts and steady state control specifications. International Journal of Mineral Processing. Elsevier; 1990;29(3):149-74.

Yingling JC. Parameter and configuration optimization of flotation circuits, part I. A review of prior work. International journal of mineral processing. Elsevier; 1993;38(1):21-40. 


\title{
Chapter 3
}

\section{Application of Linear Circuit Analysis in Evaluation of Mineral Processing Circuit Design Under Uncertainty}

\author{
(ABSTRACT)
}

This chapter describes an approach to estimate uncertainty propagation in mineral processing separation circuits. Since most modeling and simulation tools only consider deterministic input values, the inherent uncertainty induced by variable feed grade and stage unit recovery are not included in the design and optimization process. The method described in this paper combines the linear circuit analysis approach and the law of the propagation of errors. The result is a method that can be used to estimate uncertainty propagation, even in the early design stages where extensive experimental data is unavailable. To validate this approach, Monte Carlo simulations were conducted on 35 simple two, three, and four-unit circuit designs and the data was analyzed to show distribution statistics for circuit recovery, product grade, and separation efficiency. Both methods show nearly identical results, but the new methodology also gives fundamental insight on the reasons why certain circuits propagate uncertainty for specific performance indicators.

Keywords: circuit design; uncertainty; linear circuit analysis; Monte Carlo simulation; law of propagation of error.

(Note: This chapter was largely derived from the following publication and has been reformatted to departmental guidelines: Amini SH, Noble A. Application of linear circuit analysis in evaluation of mineral processing circuit design under uncertainty. Minerals Engineering. Elsevier; 2017;102:18-29.) 


\subsection{Introduction}

\subsubsection{Background}

Mineral processing and other physical separation plants often employ complex multiunit circuits to overcome the inherent inefficiencies of single unit operations. In most cases, single units are deficient in meeting final product specifications while maintaining recovery and grade at economically-favorable levels. Additionally, these single unit inefficiencies also lead to product variable and prediction uncertainty, which is then compounded by varying feed grades and slow lagging process control. Multi-unit circuits can mitigate these issues; however, the circuit design must be carefully considered, as additional units do not necessarily equate to improved performance. As a result, the optimal design of the separation circuit is a complex and open-ended engineering problem that requires a comprehensive and deliberate approach. Unfortunately, the vast number of feasible configurations as well as competing design objectives have led to an overreliance on trial-and-error and iterative approaches based on a deterministic solution (Noble 2013; Noble and Luttrell 2014). Uncertainty is typically not considered in the early design stages, even though sales contracts often specify narrow margins on final product specifications and design decisions are often based on the constraints imposed by the contract. Despite the aforementioned problems, a comprehensive solution is essential due to the demands for more profitable and efficient circuits to treat more sophisticated ore resources with diminishing feed grades (Jamett et al. 2012; Montenegro et al. 2013b). Considering the large flow volumes, high capital costs, and relative rigidity of the final flowsheet, considerable effort in the initial testing phase must guarantee that a suitable separation circuit is selected relatively early in the design process (Noble 2013; Noble and Luttrell 2014).

Prior to the advent of process modeling and simulation tools, most circuit designs were determined by heuristic methodologies and established industry practices (Lauder and McKee 1986; Wills 2011; Lucay et al. 2012). Given the low level of complexity and lack of mathematical rigor, these strategies are easy to implement and, even today, are widelyaccepted in the industry. However, since they rely on experiential rules, these classical methodologies may totally fail to achieve an optimal solution (Mendez et al. 2009; Lucay et al. 2012). Over the last 30 years, these heuristic approaches have been slowly displaced by 
numerical techniques, chiefly phenomenological process modeling and simulation (Lynch et al. 1981; King 2012). With the advent and development of these techniques, circuit selection has favored a more rigorous approach since these tools provide a means to assess circuits on the basis of one or more techno-economic performance indicators. Unfortunately, without a prescribed optimization strategy, these tools can lead to large-scale iterative trials, where several circuits are simulated repeatedly until a suitable candidate is identified.

To hasten this approach, numerous researchers have identified optimization approaches that mitigate the need for blind iterative simulation, and a comprehensive review of these strategies has been presented by Mendez et al. (2009). Most of these circuit optimization approaches use a superstructure to represent the set of alternative circuit designs. A mathematical model is then developed, and a search strategy is used to identify the optimal circuit based on one or more specified objective functions. An optimal (or pseudo-optimal) solution may be obtained by utilizing one of several search algorithms, including: genetic search algorithms (Guria et al. 2005, 2006), mixed integer programming (Cisternas et al. 2006; Jamett et al. 2015; Calisaya et al. 2016), integer programming, and other methods (Schena et al. 1996, 1997). Despite the merits of this work, contemporary industry practice still favors classical heuristic solutions. Optimally-derived circuits occasionally produce impractical results, and the optimization approaches are considerably complex. The search algorithms are strongly dependent on the input data and the accuracy of the process models. For example, Cisternas et al. (2014) has shown that the optimal circuit selection is highly dependent on the mineral market price and the specific objective function used in the analysis. Finally, since the solutions are a result of brute mathematical calculations, the optimization approach does not provide any fundamental information on what causes one circuit to be better than another.

As an alternative to the modeling, simulation, and numeric optimization design approach, linear circuit analysis (LCA) is a tool that evaluates and ranks separation circuit designs through simplified algebraic formulations (Meloy 1983). The method is particularly useful in the preliminary design stages, as it does not rely on a strict process model and thus requires limited a priori information on the equipment specifications and feed conditions. During these initial stages, detailed experimental data on circuit performance (such as lockedcycle or pilot tests) can be untimely or costly, and LCA can provide a rigorous and 
fundamental approach to guide circuit designers to an optimal solution. Nevertheless, LCA cannot completely supplant advanced simulation and circuit optimization techniques, as these approaches are still required for the final solution and performance benchmarking. LCA is fundamentally restricted by the assumption of process linearity, which specifies that a unit's partition function is not influenced by particle-particle interactions. While this assumption is not wholly valid for processing plants, several industrial applications have verified linearity in some cases via experimental investigations (Harris and Cuadros-Paz 1978; Williams and Meloy 1983; Williams et al. 1986); and the practical utility of LCA has been repeatedly demonstrated in both greenfield (Luttrell et al. 1998) and operating (McKeon and Luttrell 2005; McKeon et al. 2012) plant designs.

\subsubsection{Uncertainty Consideration in Circuit Design}

Most of the preceding circuit design methodologies, including LCA, have been constructed, implemented, and validated using a deterministic modeling approach. Nevertheless, several researchers have shown that uncertainty can be a significant factor in many mining and mineral processing applications. Kraslawski (1989) extensively reviewed various economic and technical uncertainties in chemical processing operations, including uncertainty in the final product price, feed conditions, and kinetic constants. From a mineral processing perspective, Ghaffari et al. (2012) systematically analyzed mineral assays and flow rates of several streams in a lead-zinc flotation plant. Over the 3-month period of this investigation, the plant data clearly showed the variability of different factors, such as feed grade and flow rate as well as unit recovery. Moreover, the data addressed the dramatic impact that these uncertainties have on the separation performance of the flotation circuit. Other researchers have also evaluated the influence of parameter uncertainty on the variability of flow rates in flotation circuits (Xiao and Vien 2003) and the variance of SAG mill power draw (Karamoozian et al. 2008). The summative results from these studies show that process uncertainties must be incorporated in the circuit design procedure to achieve an optimal circuit design.

Uncertainty is a broad term, and may be haphazardly applied in the context of mineral processing plant design. Generally, sources of uncertainty in physical operations may include measurement errors, systematic errors, natural variation, inherent randomness and subjective 
judgments (Morgan et al. 1992; Hoffman and Hammonds 1994; Regan et al. 2002). Simonsen and Perry (1999) proposed one method of classifying uncertainty in the mining operations by identifying the final parameter where the uncertainty is expressed. This classification identified various uncertainty classes including market price, ore reserve, mining costs, schedule duration, mining methods. Other researchers use a more mechanistic classification, which instead isolates the fundamental cause of the uncertainty as either epistemic (i.e. lack of knowledge) or stochastic/aleatory (i.e. inherent randomness that arises from natural heterogeneity) (Bárdossy and Fodor 2001; Der Kiureghian and Ditlevsen 2009; Caers 2011; Lisitsin et al. 2014; Jamett et al. 2015). Epistemic uncertainty may be reduced by further investigations; however, stochastic uncertainty may not be eliminated or even reduced in many cases. During the design stage, the inherent variability of the final product price is determined by the commodity market and, similar to the feed and ore grade parameters, represents stochastic uncertainty. Alternatively, uncertainty in the unit recovery is an epistemic uncertainty and can be varied by using different design conditions and operational parameters (Jamet et al. 2015).

While individual causes of uncertainty are significant, the circuit designer must also consider the compounded uncertainty imputed by the actual circuit design and stream connections. Current predictive modeling tools often consider static input values; however, some recent studies have investigated the impact of stochastic input data on the final separation performance for various circuit configurations (Lucay et al. 2012; Cisternas et al. 2015; Jamett et al. 2015; Montenegro et al. 2015). Since global optimization through experiments is nearly impossible, Lucay et al. (2015), Sepúlveda et al. (2014; 2015), and Lucay et al. (2012) evaluated the significance of each individual unit in determining the variability of global recovery using local and global sensitivity analysis methodologies. Jamett et al. (2012) and Jamett et al. (2015) applied stochastic programming for flotation design using a simple flotation model and analyzed the effect of uncertainty in the circuit design process. More recently, Montenegro et al. (2015) studied the influence of the statistical distributions of input data on the resultant distribution for the global recovery, while Sepúlveda et al. (2014) evaluated the importance of statistical distribution types in addition to the compounded uncertainty inherent to the circuit design. Altogether, these prior studies demonstrate the significance of uncertainty in the circuit design problem and highlight the 
need for better fundamental understanding on the mechanisms that cause uncertainty propagation in mineral processing circuits.

\subsubsection{Objectives}

Given the complexity of the current optimization strategies for mineral processing circuit design, this chapter presents a novel approach to uncertainty quantification based on LCA. In summary, the following objectives are identified:

- Review the LCA approach and derive a means to quantify uncertainty using LCA and the law of propagation of error principles;

- Compare the results of the simplified law of propagation of error approach to the more rigorous Monte Carlo approach in quantifying uncertainty in circuit recovery, separation efficiency, and grade.

- Prescribe a means to apply the new method in practical applications.

\subsection{Theory}

\subsubsection{Linear Circuit Analysis Review}

Linear circuit analysis (LCA), as a circuit evaluation technique, is employed to assess the separation performance of multi-unit circuits only based on unit arrangements and stream interconnections (Meloy 1983). The LCA methodology is formulated based on separation probabilities. For any separation unit, the concentrate-to-feed mass flow ratio $(C$ and $F$, respectively), may be given for any particle class as a function of the equipment characteristics and the physical properties of the individual particles. This ratio is often denoted the separation probability $(P)$, the unit partition function, or the unit transfer function. For the LCA approach, an explicit function for $P$ is not required, and instead, the implicit characteristics of such a function are carried through the analytical steps. For example, each unit with a generic transfer function of $P_{i}$, produces a concentrate product, $C=F\left(P_{i}\right)$, and a tailing product, $T=F\left(1-P_{i}\right)$. By extending the simplified algebra, the final circuit concentrate 


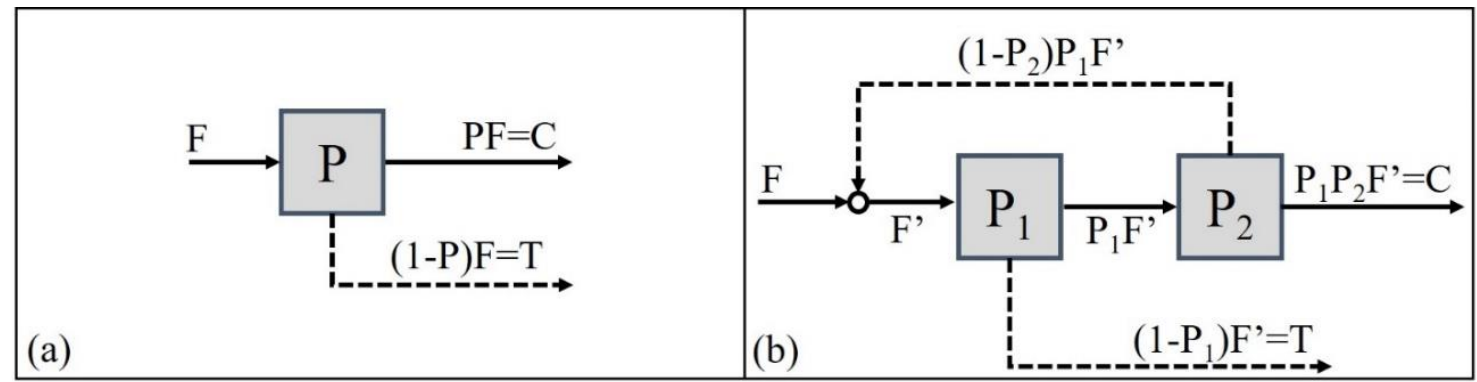

Figure 3-1: Linear circuit analysis formulation examples; (a) individual unit circuit and (b) multistage circuit.

and overall circuit transfer function $(C / F)$ may be determined for any circuit of arbitrary size in terms of different $P$ values.

The fundamentals of LCA are well-illustrated using series of simple examples. Consider the single-unit operation shown in Figure 3-1a. By defining the unit's feed, concentrate, and tailings mass flow rates as $F_{n}, C_{n}$, and $T_{n}$, respectively, the mass balance for any particle class around a single unit is given by:

$$
\begin{aligned}
& \mathrm{C}_{n}=F_{n} P_{n} \\
& T_{n}=F_{n}\left(1-P_{n}\right)
\end{aligned}
$$

This type of analysis can be extended to multi-unit separations by algebraically deriving a function to describe the circuit concentrate-to-feed ratio $(C / F)$ as a function of individual unit separation probability $\left(P_{i}\right)$ functions. The final circuit transfer function expression is defined as the analytical circuit solution, as follow:

$$
C / F=f\left(P_{1}, P_{2}, \ldots, P_{i}\right)
$$

As shown in Figure 3-1b, the final analytical solution for a two unit recycle circuit can be expressed as: 


$$
C / F=\frac{P_{1} P_{2}}{P_{1} P_{2}-P_{1}+1}
$$

This equation indicates that the numeric circuit recovery value $(C / F)$ can be directly calculated for any particle class, if the individual partition functions for the two units are known explicitly. Once recovery for each particle class is determined, other performance indicators, such as gangue rejection, product grade, and separation efficiency can be readily determined for a given ore feed distribution.

Detailed descriptions and applications of these techniques have been thoroughly explained in the literature and include: original LCA derivation and separation sharpness (Meloy 1983); streamlined analytical solution derivation (Noble 2013; Noble and Luttrell 2014); and techno-economic separation efficiency and yield determination (Noble and Luttrell 2015). Altogether, these calculations bring considerable value to the LCA approach, since they permit relative comparisons solely from the stream interconnections. Since the technique does not require information on feed conditions or equipment, LCA provides means to determine the fundamental characteristics of stream interconnections that lead to improved performance. Prior works have used LCA to demonstrate the role of various features in circuit design including: recirculating loads (Meloy 1983), fundamental processing functions (Williams and Meloy 1989), cut point shifts (Meloy et al. 1986), and dynamic circuit stability (Williams and Meloy 1983).

\subsubsection{Monte Carlo Simulation Review}

Monte Carlo Simulation (MCS, or stochastic simulation) refers to a numerical and probabilistic analysis technique which is based on (pseudo) random sampling from a domain of input parameters with a known statistical distribution. This random sampling is repeated a "large" number of times, and given enough iterations, the MCS method allows one to quantitatively evaluate uncertainty and variability in risk assessments by determining the distribution of expected outcomes from a standard deterministic process model. MCS has been implemented in a variety of business, technical, and scientific domains, and details on 
how to implement MCS are well-documented in a number of texts, including Mooney (1997), Thomopoulos (2012), and Zio (2013).

While business and financial applications have seen the most widespread use of MCS (e.g. value projects, options and portfolios), mineral and energy industry practitioners have also experimented with its use, particularly in hydrocarbon reservoir analysis (Bosch et al. 2007), mineral reserve estimation (Dimitrakopoulos 1998), material balance problems (Perry 1997), process simulation (Merks 1999), analysis of uncertainty in mineral processing (Xiao and Vien 2003), and, to some extent, evaluation and optimization of process circuit design (Montenegro et al. 2013a, 2013b, 2015). While many of the prior papers have shown the use of MCS in isolated problems, the recent paper by Montenegro et al. (2015) has demonstrated the value of MCS in large-scale simulation problems that consider multiple uncertain input parameters and the influence that the input distribution type, uncertainty level, and circuit type have on the final output distribution. Furthermore, Xiao and Vien (2003) compared the uncertainty determined from MCS to that estimated by the law of propagation of error and concluded that MCS was a superior method in most cases. While the overall results of these studies conclude that MCS is an appropriate tool to analyze and determine uncertainty propagation in mineral processing applications, the latter paper by Xiao and Vien (2003) will be discussed in more detail in the next section to reassess the appropriate value of the propagation of error method.

In the current chapter, MCS is used to numerically determine distributions of process performance indicators (i.e. circuit grade, recovery, and separation efficiency) when given a specific circuit design and distributions of input feed grade and recovery values for single unit operations. At a macroscopic level, nearly all of the uncertainty in the circuit design problem arises from distributed inputs for the circuit feed grade and numerous operational parameters that are ultimately reflected in the recovery values of individual units. As a result, for the purpose of comparative evaluation of circuit configurations, a suitable MCS approach needs to only consider these parameters as stochastic inputs, rather than the individual factors that contribute to unit recovery. Furthermore, this approach (which focuses strictly on comparative evaluation of circuit designs) negates the need for a unit operation process model, which may also be subject to further uncertainty. Ultimately, if the input distribution for unit recovery and feed grade are known, the output distribution of the circuit 
performance indicators can be readily determined by MCS. These results can then be analyzed to determine how the circuit design influences circuit uncertainty (i.e. output distribution width), when given the same distribution for the input parameters. While mineral price and other economic factors are also significant distributed parameters, they have been excluded from this analysis to rather focus on the technical components of uncertainty propagation.

\subsubsection{Derivation of an Uncertainty Evaluation Method Based on Linear Circuit Analysis/Law of Propagation of Error}

As described above, the separation performance of each mineral processing circuit can be specified using a unique analytical transfer function that can be derived from LCA. This function has considerable value in assessing separation performance and explaining the fundamental circuit characteristics that modify separation performance. As an extension of these prior LCA methods, this algebraic formulation may be employed to characterize the uncertainty or distribution width inherent to a particular circuit configuration using the propagation of uncertainty principle (or propagation of error, LPE). This method is distinct from the MCS approach as it does not require iterative simulation and, in principle, could be performed using a simple hand calculator. Second, since the results are given in an analytical, rather than numeric form, a more fundamental analysis can be performed to determine the causal factors for circuit uncertainty.

LPE is used to determine the statistical impact of the uncertainty that one or more input variables have on a function evaluation. The uncertainty of a quantity is often quantified as the standard deviation $(\sigma)$ of the value's expected distribution. If the function of $F(X, Y, Z)$ is continuous and has continuous derivatives of the first and second order with respect to its components, and further $X, Y, Z$ are independent (i.e. the covariance of any two components are zero), the standard deviation of $F$ may be estimated by the LPE rule, given by:

$$
\sigma_{F}=\sqrt{\left(\frac{\partial F}{\partial X}\right)^{2} \sigma_{X}^{2}+\left(\frac{\partial F}{\partial Y}\right)^{2} \sigma_{Y}^{2}+\left(\frac{\partial F}{\partial Z}\right)^{2} \sigma_{Z}^{2}}
$$


where $\sigma_{F}, \sigma_{X}, \sigma_{Y}$, and $\sigma_{Z}$ are the standard deviation of function (F) and input variables $(\mathrm{X}, \mathrm{Y}, \mathrm{Z})$; and $\left(\frac{\partial F}{\partial X}\right),\left(\frac{\partial F}{\partial Y}\right)$, and $\left(\frac{\partial F}{\partial Z}\right)$ are the derivatives of the function $\mathrm{F}$ with respect to $\mathrm{X}, \mathrm{Y}$, and $\mathrm{Z}$.

The LPE may be applied to quantify the uncertainty in the circuit recovery of specific particle classes using circuit analytical transfer functions determined from LCA. Each individual P (i.e. stage recovery value) value constitutes an input parameter, and thus, the LPE calculation only requires the partial derivative of the circuit analytical function with respect to each $\mathrm{P}$ value. This general formulation is given by:

$$
\sigma_{(C / F)}=\sqrt{\left(\frac{\partial(C / F)}{\partial P_{1}}\right)^{2} \sigma_{P_{1}}^{2}+\left(\frac{\partial(C / F)}{\partial P_{2}}\right)^{2} \sigma_{P_{2}}^{2}+\left(\frac{\partial(C / F)}{\partial P_{3}}\right)^{2} \sigma_{P_{3}}^{2}+\ldots} .
$$

As is typical for LCA analyses, the problem can be reduced to two dimensions by assuming each $P$ value to be equal. After this simplification, Equation 5 reduces to:

$$
\sigma_{(C / F)}=\sqrt{\left(\frac{\partial(C / F)}{\partial P}\right)^{2} \sigma_{P}^{2}}
$$

After simplification, $\sigma_{(C / F)}$ is simply given by the local derivative of the circuit transfer function at the desired $\mathrm{P}$ value multiplied by the inherent uncertainty of that parameter. Mechanistically, this equation can be readily applied to any circuit of arbitrary size to determine the relative standard deviation or output uncertainty of the circuit recovery value for a specific particle class. The only inputs that are needed are the circuit transfer function $(\mathrm{C} / \mathrm{F}$, determined from LCA), the approximately recovery value in each stage for the designated particle class ( $\mathrm{P}$, can be estimated a priori), and the inherent uncertainty for that recovery in single unit operations ( $\sigma$, can be readily estimated from historic data). All of these values are readily available to the circuit designer even in the early design stages. 


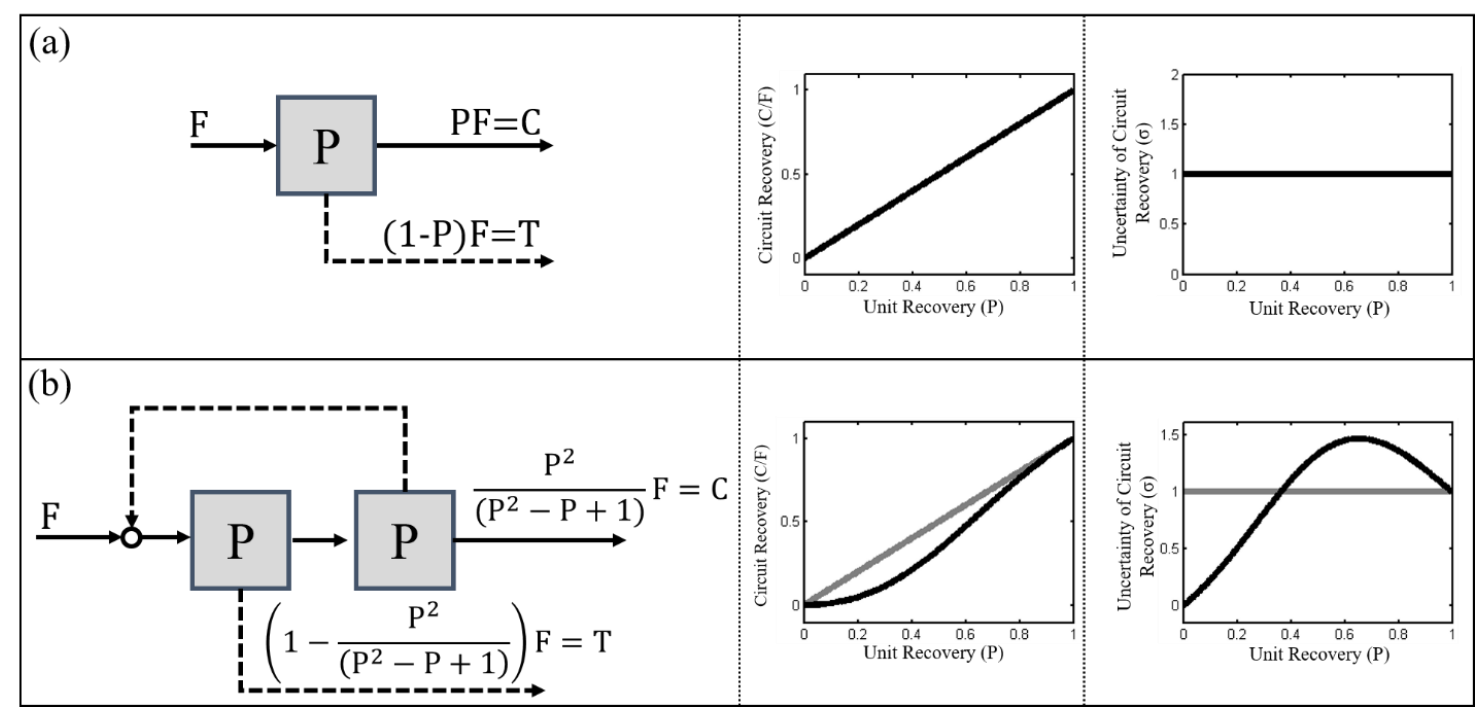

Figure 3-2: Uncertainty quantification using linear circuit analysis formulation and propagation of error concept; (a) single unit circuit and (b) multistage circuit. Middle panels show global circuit recovery as a function of unit recovery as determined from

$$
\text { LCA. }
$$

While the constant P simplification is obviously not true for operating circuits, it does provide a general means to rank circuits and expose fundamental relationships. It also provides a convenient method of graphical analysis. Nevertheless, this simplification does not preclude the method from more sophisticated multi-dimensional analysis, if the information can be estimated during the circuit design. This simplification also indicates, at a minimum, the approximate recovery value and the deviation of that recovery value are needed to perform an LCA uncertainty analysis.

Figure 3-2 continues the prior LCA analysis to include the uncertainty component. In this example, all $\mathrm{P}$ values have been assumed equal to simplify the analysis. The middle panel shows the circuit transfer function $(\mathrm{C} / \mathrm{F})$ as a function of unit $\mathrm{P}$ values as determined from LCA. As a matter of interpretation, these unit $\mathrm{P}$ values are analogous to particle classes, as in an operating circuit, an individual particle will have a characteristic P value under specific operation conditions. This graph can be interpreted to show whether the recovery of that particle class is increased or decreased when compared to a single unit. While the single unit case is trivial, the cleaner circuit in the lower panels shows that for all unit P values, the circuit 
recovery is reduced (i.e. a particle that has 0.20 recovery in a single unit will only have 0.04 recovery in the circuit).

This analysis is extended in the right panel of Figure 3-2, which shows the derivative of this function as a function of the unit $\mathrm{P}$ value. As described above, the first derivative evaluated at a given point is analogous to the uncertainty propagation in that point (assuming a constant, non-zero value for the unit standard deviation, $\sigma$ ). Once again, the single unit case is trivial, as the circuit uncertainty remains constant through all P values. Alternatively, the cleaner circuit includes values above and below 1.0, indicating that the circuit has the ability to suppress or intensify uncertainty in circuit recovery, depending on the unit P values. For example, at a unit $\mathrm{P}$ value of 0.20 , the circuit uncertainty $\left(\sigma_{\mathrm{C} / \mathrm{F}}\right)$ is 0.50 . This value indicates that the distribution of possible recovery values will be narrower than that of a single unit, when both are given the same input distribution. Alternatively, at a P value of 0.8 , the circuit uncertainty is 1.37 . Here the distribution will be wider for the circuit, thus implying more uncertainty in the possible results or a wider range of possible results. Stated simply, the shape of the derivative curve indicates that this cleaner will increase uncertainty on the recovery of ore particles and reduce uncertainty on the recovery of gangue particles.

While the theoretical development has focused on unit recovery uncertainty, a similar approach can be used to determine the uncertainty in other circuit performance indicators such as grade and separation efficiency. Algebraic functions for these parameters can also be readily derived for any circuit when given the circuit transfer function and information on the inventory of various particle classes.

\subsection{Methodology and Data Analysis}

\subsubsection{Overall Approach}

The purpose of this study is to determine the utility of the LPE-LCA methodology in evaluating uncertainty in mineral processing circuit configurations. Since different circuit designs are expected to expand and contract the distribution of outcomes, the LPE-LCA methodology may provide a simple and straightforward approach to estimate these changes for any circuit configurations when given limited input data. 
This evaluation was conducted by first simulating several generic one, two, three, and four-unit circuits using MCS with a specified distribution of various input parameters. For simplicity, only two components (valuable and gangue) were considered in these simulations, and the variation in plant operational parameters was simplified to be reflected solely in the unit recovery. Global recovery, product grade, and separation efficiency were then calculated for each simulation, and the results were compiled by determining the mean and moments of the resultant distribution.

To analyze the uncertainty of each separation performance factor, the standard deviation of each population was determined. For comparison, the single unit was considered the reference case, and all standard deviation values for multi-unit circuits were evaluated as a ratio to this single unit case. As a result, a new uncertainty metric, "relative uncertainty", is defined to compare the variability induced by different circuit designs relative to the variability in a single unit.

Parallel to this MCS study, LPE-LCA was also used to predict the relative uncertainty of the various performance indicators (recovery, grade, and separation efficiency) solely from the circuit design using Equation 5, and the respective circuit transfer functions. These two independent results finally compared at different levels of mean and standard deviation values to assess the relevance of the LPE-LCA methodology.

\subsubsection{Circuits \& Data Analysis}

The various measures and comparisons of uncertainty were performed on generic circuit configurations covering a range of standard design templates. Figure 3-3 shows schematic representations of these circuits as well as the circuit transfer functions given in terms of unique unit transfer functions. While not completely exhaustive, the two- and three-unit circuits represent the most commonly utilized base configurations for many separation processes and provide a basis for comparative analysis. In addition, the four-unit circuits validate the methodology for reasonably complex alternatives. 


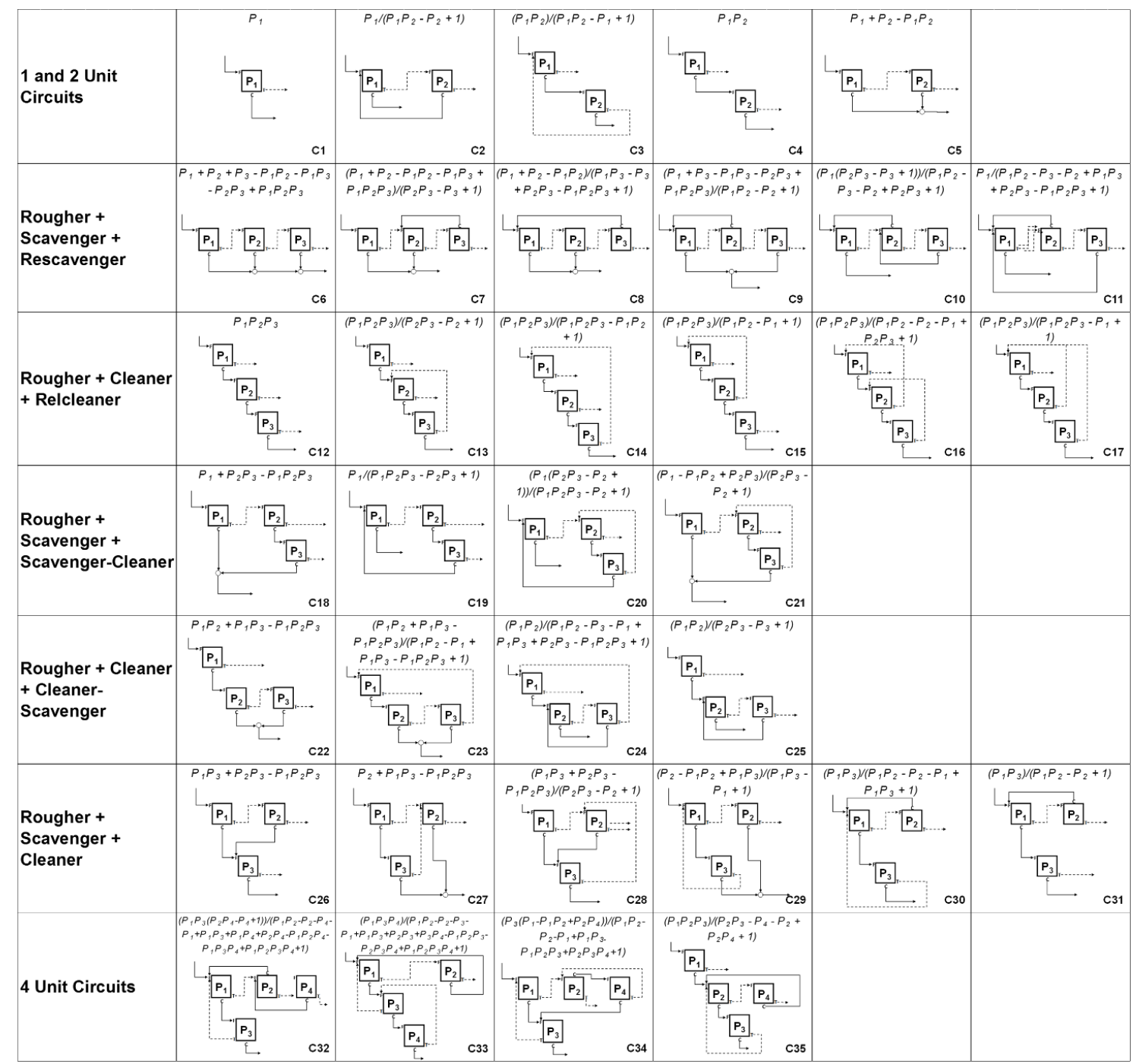

Figure 3-3: Basic circuit drawings and analytical solutions.

When given numeric values for unit recovery and feed grade, the circuit transfer functions in Figure 3-3 can be used to determine global recovery of each component. For example, the global recovery of valuable material in circuit $\mathrm{i},(C / F)_{i}^{V}$, is given by:

$$
R_{i}^{V}=\left(\frac{C}{F}\right)_{i}^{V}=f\left(P_{1}^{V}, P_{2}^{V}, \ldots, P_{N}^{V}\right)
$$


where $P^{V}$ is the recovery of valuable particles in a single unit. The subscript here refers to the individual units in the circuit (e.g. 1 = rougher, 2 = scavenger, $3=$ cleaner, etc.).

Global recovery of gangue materials is defined similarly:

$$
R_{i}^{G}=\left(\frac{C}{F}\right)_{i}^{G}=f\left(P_{1}^{G}, P_{2}^{G}, \ldots, P_{N}^{G}\right)
$$

where $P_{n}^{G}$ is the recovery of gangue in the nth unit.

Global separation efficiency of circuit is defined as the difference between global recovery of valuable and gangue materials:

$$
S E_{i}=R_{i}^{V}-R_{i}^{G}
$$

Finally, concentrate grade of circuit i $\left(G_{i}^{C}\right)$ can be determined from the global valuable and invaluable recovery as well as the feed grade $\left(G^{F}\right)$ :

$$
G_{i}^{C}=\frac{G^{F} \times R_{i}^{V}}{\left(G^{F} \times R_{i}^{V}\right)+\left(\left(100-G^{F}\right) \times R_{i}^{G}\right)}
$$

\subsubsection{LPE/MCS Comparison}

MCS was executed to evaluate the behavior of 35 generic one, two, three, and four-unit circuits when considering normally distributed feed grade and individual unit recovery values as input data. Table 3-1 shows the initial assumptions that were used to construct various sets of random numbers for feed grade and unit recovery parameters. As discussed earlier, for the purpose of the current work, the recovery of different classes of particles at various separation stages (i.e. rougher, cleaner, scavenger, and etc.) initially considered similar and subsequently relative uncertainty is determined when different unit recovery values are considered for distinct individual units. For each circuit, 1,000,000 discrete simulations were conducted, and Equation 7 to 10 were used to determine performance indicators. 
Results were compiled into the distributions, and these distributions were analyzed for mean and standard deviation values.

Following the MCS study, the LPE equation was applied to each analytical circuit solution to calculate the relative uncertainty of global recovery using Equation 6. For further investigation, to determine the relative uncertainty of separation efficiency and product grade factors, the following formulations may be utilized considering the fundamental of LCA and LPE concepts:

$$
\sigma_{\left(S E_{i}\right)}=\sqrt{\left(\frac{\partial\left(S E_{i}\right)}{\partial R_{i}^{V}}\right)^{2} \sigma_{R_{i}^{V}}^{2}+\left(\frac{\partial\left(S E_{i}\right)}{\partial R_{i}^{G}}\right)^{2} \sigma_{R_{i}^{G}}^{2}}
$$

where $\sigma_{\left(S E_{i}\right)}, \sigma_{R_{i}^{V}}$, and $\sigma_{R_{i}^{G}}$ are the standard deviation of separation efficiency, recovery of valuable materials, and recovery of gangue materials in circuit $\mathrm{i}$.

$$
\sigma_{\left(G_{i}^{C}\right)}=\sqrt{\left(\frac{\partial\left(G_{i}^{C}\right)}{\partial G^{F}}\right)^{2} \sigma_{G^{F}}^{2}+\left(\frac{\partial\left(G_{i}^{C}\right)}{\partial R_{i}^{V}}\right)^{2} \sigma_{R_{i}^{V}}^{2}+\left(\frac{\partial\left(G_{i}^{C}\right)}{\partial R_{i}^{G}}\right)^{2} \sigma_{R_{i}^{G}}^{2}}
$$

where $\sigma_{\left(G_{i}^{C}\right)}$ and $\sigma_{G^{F}}$ are the standard deviation of concentrate grade and feed grade, respectively.

Table 3-1: Statistical measures for feed grade and recovery of different class of particles.

\begin{tabular}{|l|l|l|}
\hline Parameter & Mean $(\mu)$ & Standard Deviation $(\sigma)$ \\
\hline Feed Grade $\left(G_{i}^{C}\right)$ & $25 \%$ & $2.5 \%$ \\
\hline Recovery of Valuable Materials $\left(R_{i}^{V}\right)$ & $75 \%$ & $7.5 \%$ \\
\hline Recovery of Gangue Materials $\left(R_{i}^{G}\right)$ & $25 \%$ & $2.5 \%$ \\
\hline
\end{tabular}




\subsection{Results and Discussions}

\subsubsection{MCS Results}

The results of the MCS study demonstrate the contrasting behavior of different separation circuits under similar uncertain conditions. For example, Figure 3-4 shows abridged results obtained from the first 1,000 iterations for rougher+scavenger+rescavenger (C11), rougher+cleaner+recleaner (C17), rougher+scavenger+scavenger-cleaner (C20), rougher+cleaner+cleaner-scavenger (C24), and rougher+scavenger+cleaner (C30) for both recovery vs. grade (left panel) and separation efficiency vs. grade (right panel). These data indicate that circuit evaluation and selection based only on one iteration (i.e. a deterministic approach) may result in the misleading conclusion. For instance, the mean values for C17 indicate superior performance from all performance indicators, and some iterations include some of the highest values for all circuits included in this figure. However, other iterations in the $\mathrm{C} 17$ dataset also include some of the lowest values for separation efficiency and grade.

Alternatively, other circuit designs (e.g. C11 and C20) produce more consistent results (low level of variability), even though they may rank low in terms of selectivity. While this example includes just a subset of the data, it is indicative of the holistic results found from the evaluation of all 35 circuits.

Figure 3-5 shows the relative uncertainty of the circuit recovery for the valuable $(\mathrm{P}=$ $75 \%)$ and gangue particles $(\mathrm{P}=25 \%)$. As mentioned above, these values were calculated from the standard deviation found from MCS, and the deviation for multi-unit circuits has been normalized to the deviation of a single unit circuit. In addition, to demonstrate the transition between these two extremes, a third particle class $(\mathrm{P}=50 \%)$ was also added to this plot, even though it was not used in any of the other analyses. Overall, Figure 3-5 demonstrates the natural trend of different circuit designs (and similar design groups) to suppress or increase the uncertainty associated with circuit recovery. This data shows that both the circuit design and the particle class recovery value significantly influence circuit recovery uncertainty. For

instance, rougher+cleaner+recleaner circuits (C12 to C17) and rougher+cleaner+recleaner+cleaner-scavenger (C35) drastically increase the level of recovery uncertainty in valuable particles, while they reduce the uncertainty in the recovery of gangue 

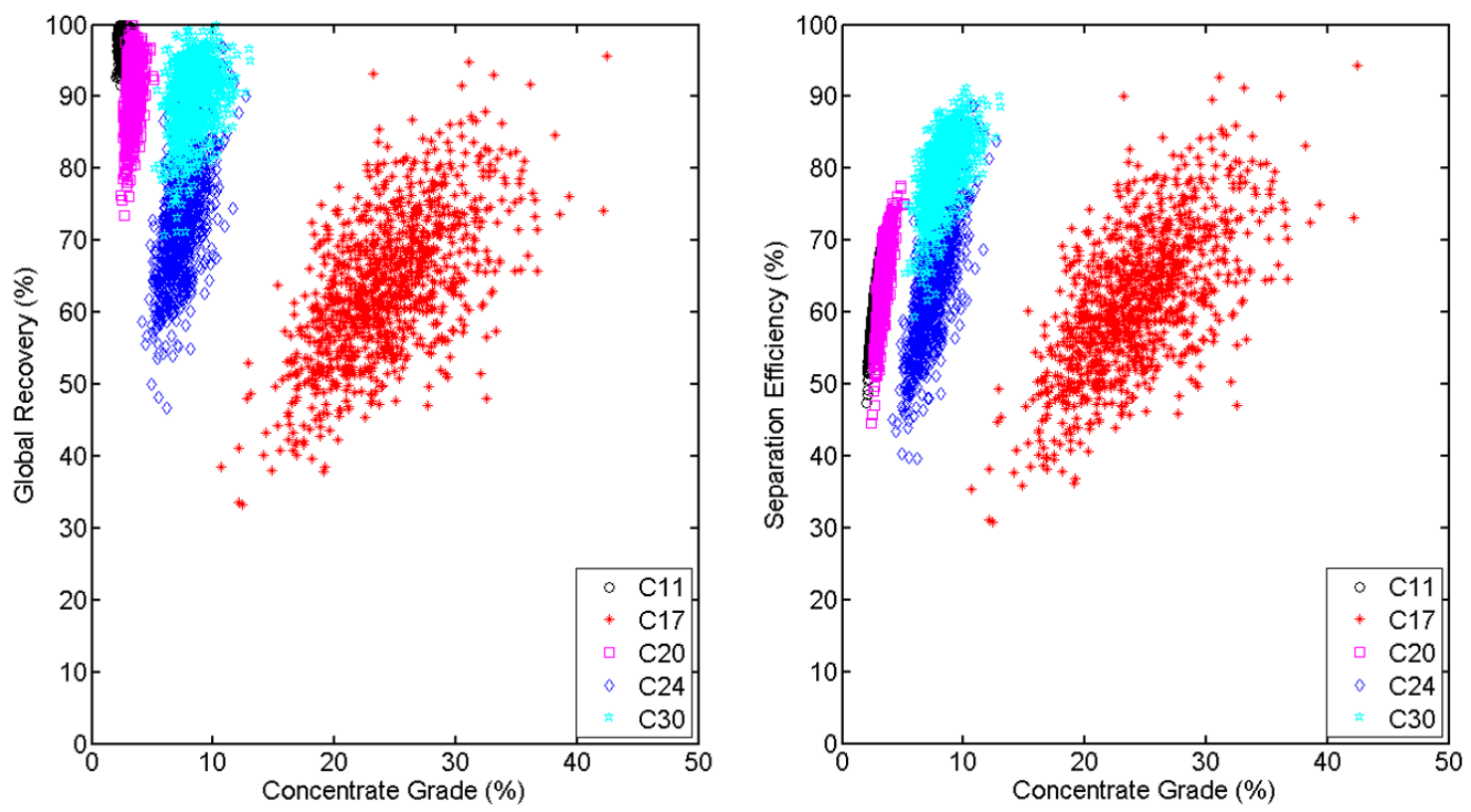

Figure 3-5: Recovery-grade (left panel) and separation efficiency-grade (right panel) plots obtained from the first 1,000 iterations of MCS for circuits number 11, 17, 20, 24, and 30.
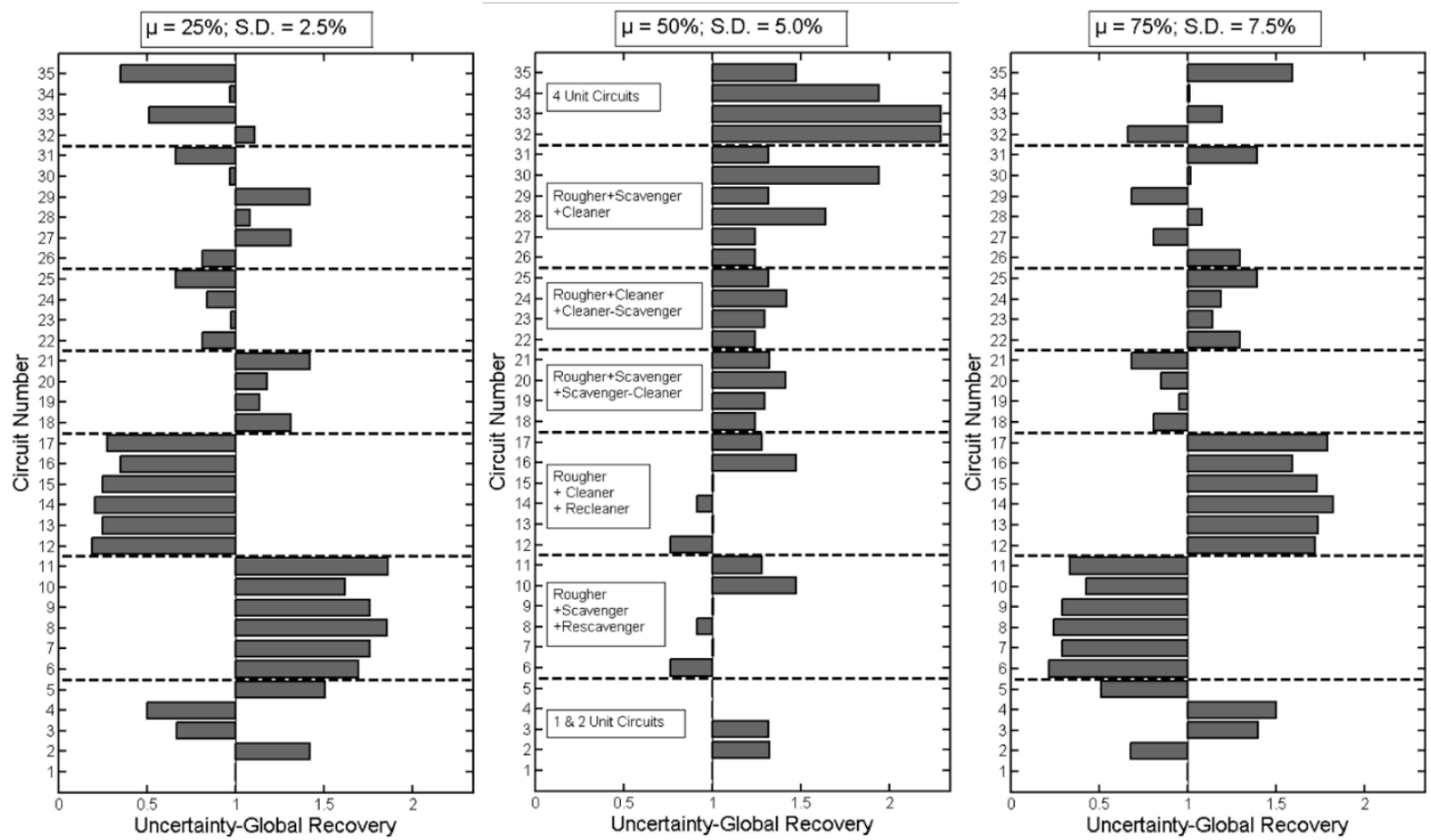

Figure 3-4: Role of different circuit configurations in surpass or inhibit the uncertainty of global recovery for different classes of materials using MCS. 

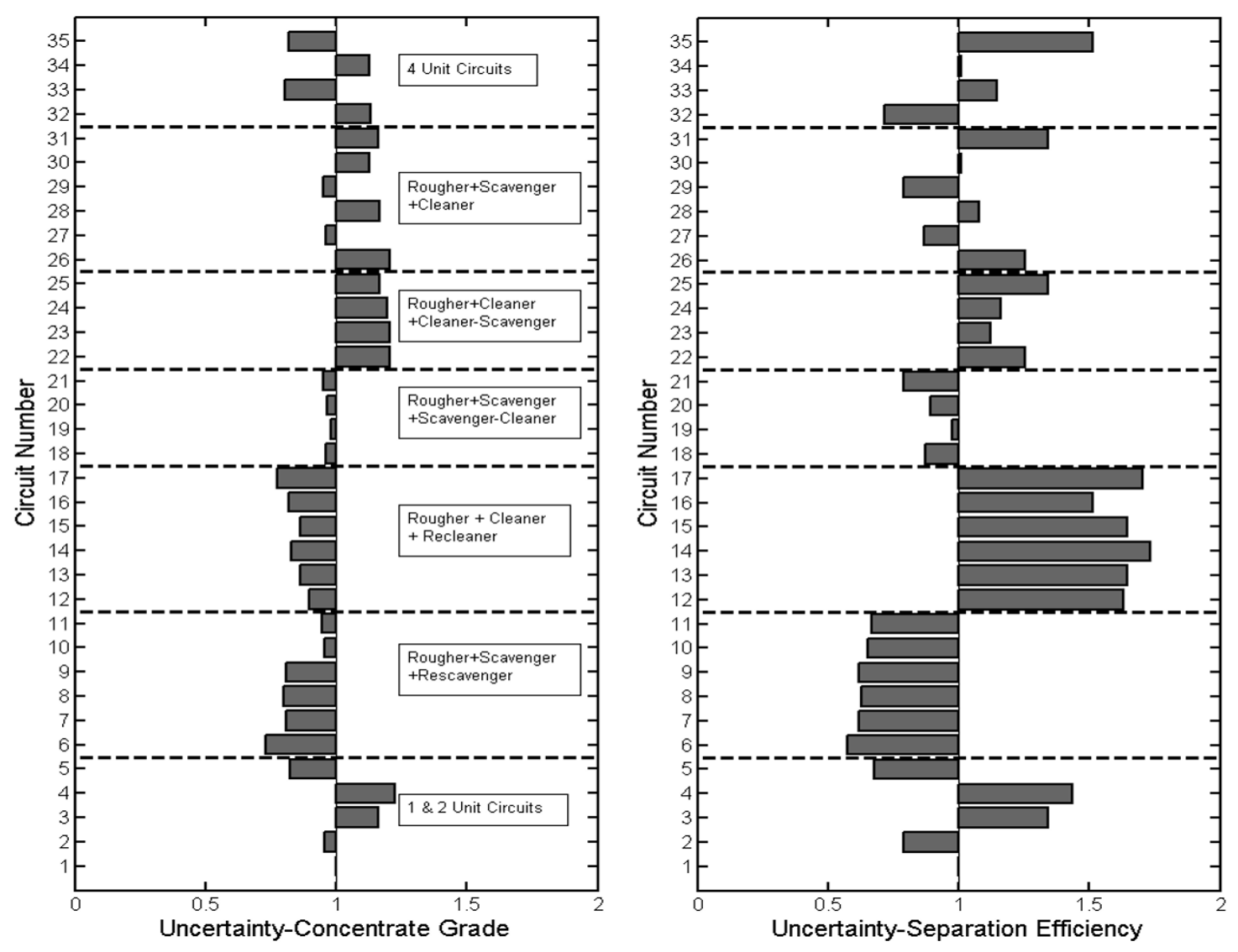

Figure 3-6: Performance of different circuit designs in surpassing or inhibiting the uncertainty of separation efficiency and concentrate grade utilizing MCS.

particles. This behavior is directly opposed to the behavior of rougher+scavenger+rescavenger (C6 to $\mathrm{C} 11$ ) circuits and rougher+cleaner+scavenger+rescavenger (C32) circuit which behave in an opposite fashion. Alternatively, the balanced circuits (such as C30) tend to show similar uncertainty for the valuable and gangue particles, but they pass through a peak for the middling or $\mathrm{P}=50 \%$ particles.

Similar observations are shown for the relative uncertainty of separation efficiency and concentrate grade in Figure 3-6. Here, "rougher+scavenger+scavenger-cleaner" circuits (i.e. C18 to C21) reduce the variability in concentrate grade while minimizing the level of relative uncertainty of the separation efficiency factor. On the contrary, circuit designs containing three units of rougher, cleaner and cleaner scavengers (C22 to C25), drastically 
increase the degree of product grade variability at the high level of relative uncertainty for the separation efficiency indicator. When taken together, the product grade data appears to behave independently from any of the other recovery parameters, and few circuits considerably reduce the uncertainty in product grade. Alternatively, the uncertainty in the separation efficiency parameter correlates very well $(\rho=0.9882)$ to the recovery of the valuable component (Figure 3-6, right panel). This behavior indicates that for the given conditions of feed grade and recovery values, separation efficiency is predominantly influenced by valuable material recovery. Altogether, these data show that different circuit configurations exert contradiction impacts on the predicted relative uncertainty under similar conditions, and a circuit design may exhibit completely different behaviors under various uncertain conditions.

\subsubsection{LPE Results}

As discussed in the theory section, comprehensive circuit evaluations may be carried out based on the fundamental of LCA-LPE concept using analytical circuit solutions. Figure 37 shows the relative uncertainty of global recovery (first derivative of analytical circuit formulation) for all 35 circuit designs as a function of unit recovery values from 0 to 1 . These data may be compared to that in Figure 3-5 to validate the LCA-LPE methodology. For example, as mentioned above, predominantly cleaning circuits (C12 to $\mathrm{C} 17$ and $\mathrm{C} 35)$ show a progressively increasing relative uncertainty as the unit recovery values pass from $25 \%$ to $50 \%$ to $75 \%$. Figure 3-7 reflects this trend, as the LCA-LPE graph for these circuits is consistently increasing within that range. Similar behavior between the two methods can also be observed for the predominantly scavenging circuits (C6 to $\mathrm{C} 11$, which are consistently decreasing) and the balanced circuits (C30, which passes through an optimum). Furthermore, the LCA-LPE analysis can provide fundamental insight and otherwise hidden trends that were not observed in the MCS study that was only conducted at discrete recovery points. For example, many curves pass through a positive optimum at some point between 0 and 1 , indicating that all circuits have limit to the amount of uncertainty that can be increased, and that limit is relative to a particular particle class. Alternatively, and

interestingly, very few circuits pass through negative optimum other than at the periphery of the curve (either 0 or 1 ). 

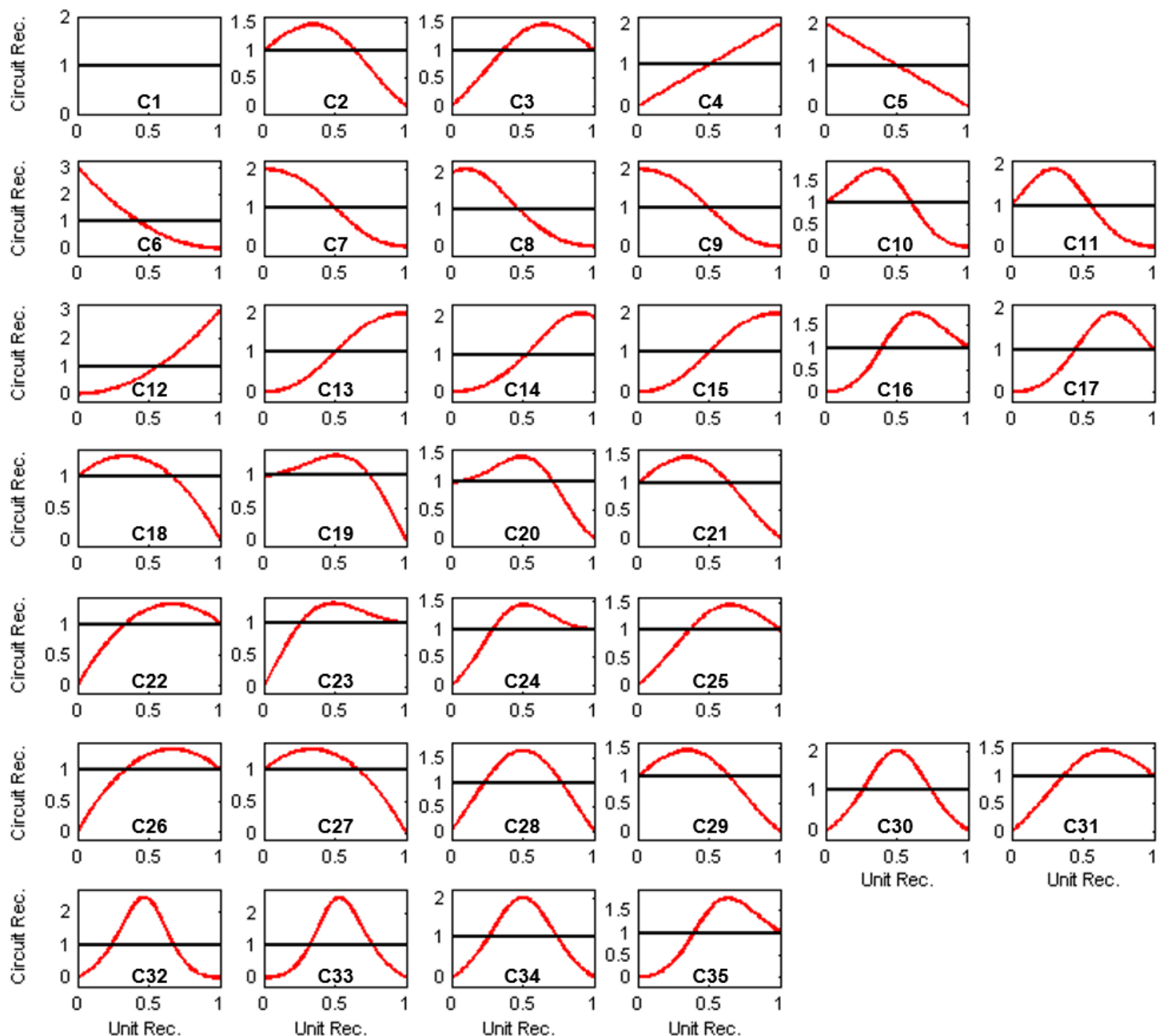

Figure 3-7:Quantification of inherent uncertainty of circuit recovery using derivative of circuit partition functions for circuit designs composed of the units with similar transfer functions.

Additionally, a quantitative validation is provided in Figure 3-8 that shows the relative uncertainty for the various circuits and various performance indicators determined from MCS plotted against the same values determined from LPE. In all cases, the two methods produce nearly identical results as indicated by coefficient of variation of $0.995,0.993$, and 0.977 , for ore recovery, concentrate grade, and separation efficiency, respectively. 

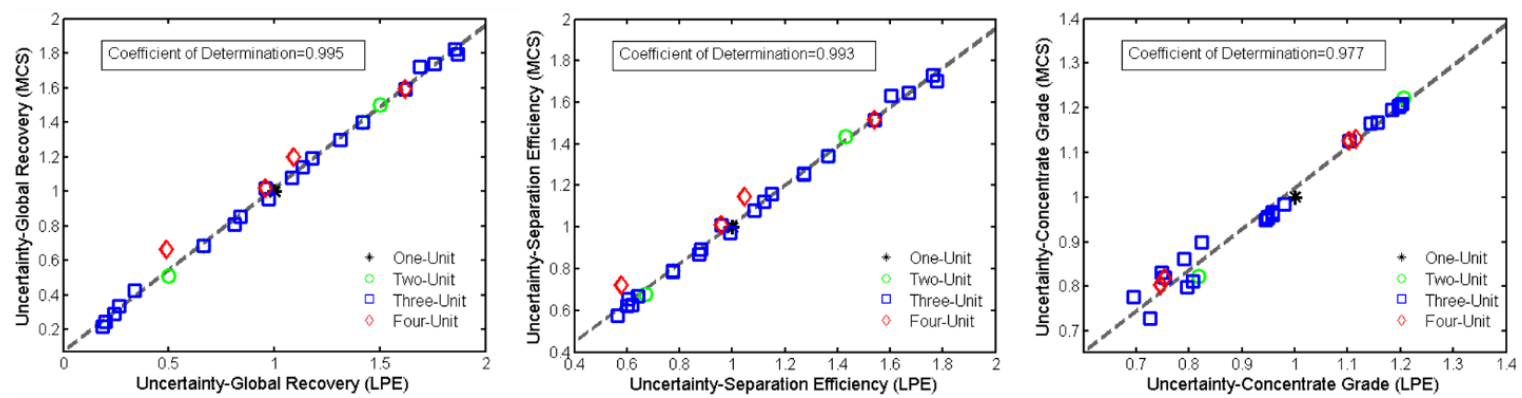

Figure 3-8: Comparison of uncertainty in circuit recovery, separation efficiency, and concentrate grade as determined from LPE and MCS methodologies. Data includes evaluations for the 35 circuits included in Figure 3-7 and input data included in Table 3-1.

(a)

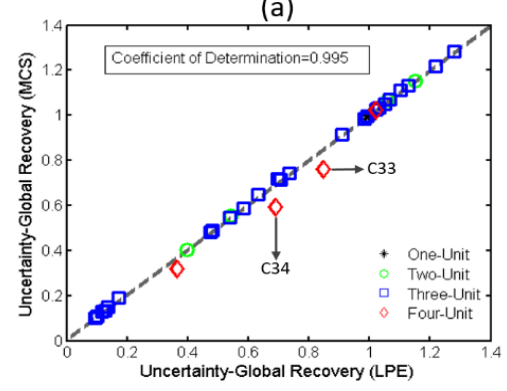

(b)

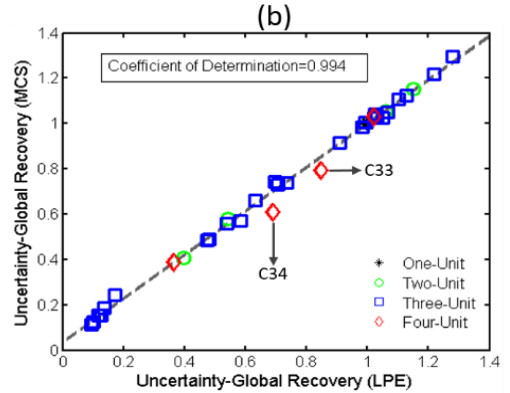

(c)

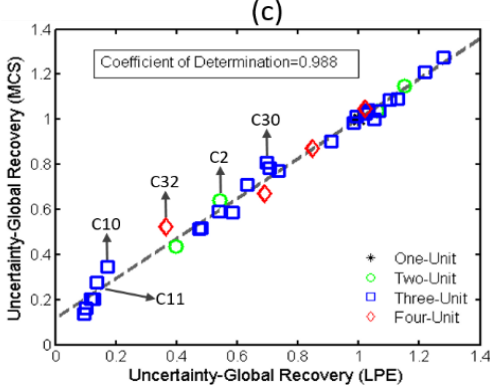

Figure 3-9: Comparisons of results obtained from LPE and MCS employing different values of unit recovery with standard deviation of $10 \%$ (a), $20 \%$ (b), and $30 \%$ (c) of mean values.

\subsubsection{Discussion and Analysis of Input Parameter Variance}

While the current results show excellent similarity between MCS and LPE results, the prior work by Xiao and Vien (2003) suggests that LPE and MCS may give divergent results, especially for complex or nonlinear cases. In general, the LPE formulation is derived using a Taylor series while ignoring third order terms and higher, and the LPE formulation assumes independence between input parameters. Due to these factors, Xiao and Vien (2003) concluded that the LPE is only an approximation and precision of the results are strongly related to the function linearity and inputs variations. Both Xiao and Vien (2003) and Karamoozian et al. (2008) have approximated the compounded uncertainty of several nonlinear system with dependent input variables employing LPE and MCS. Comparisons 
Table 3-2: Statistical measures assumptions for ore recovery parameter in different separation units.

\begin{tabular}{|c|c|c|c|c|}
\hline \multirow{2}{*}{ Parameter } & \multirow{2}{*}{ Mean } & \multicolumn{3}{|c|}{ Standard Deviation } \\
\cline { 3 - 5 } & & 1 & 2 & 3 \\
\hline Rougher & $70 \%$ & $7.0 \%$ & $14.0 \%$ & $21.0 \%$ \\
\hline Scavenger & $80 \%$ & $8.0 \%$ & $16.0 \%$ & $24.0 \%$ \\
\hline Cleaner & $75 \%$ & $7.5 \%$ & $15.0 \%$ & $22.5 \%$ \\
\hline Rescavenger & $85 \%$ & $8.5 \%$ & $16.0 \%$ & $25.5 \%$ \\
\hline Recleaner & $70 \%$ & $7.0 \%$ & $14.0 \%$ & $21.0 \%$ \\
\hline Scavenger-Cleaner & $75 \%$ & $7.5 \%$ & $15.0 \%$ & $22.5 \%$ \\
\hline Cleaner-Scavenger & $80 \%$ & $8.0 \%$ & $16.0 \%$ & $24.0 \%$ \\
\hline
\end{tabular}

between the LPE and MCS results have indicated that MCS determined more accurate results in non-linear systems where the assumption of independent inputs is not valid.

In the current paper, both MCS and LPE approaches were implemented to estimate the projected uncertainty of various circuit transfer functions. These analytical transfer functions meet the assumption of independent input parameters; however, few circuits may meet the linearity criteria. Considering the current outcome and the conflicting results presented by Xiao and Vien (2003), a secondary analysis was conducted to evaluate the precision of the LPE results with respect to different levels of input variance. In this study, the recovery uncertainty of the 35 circuits was determined using distinct unit recovery values $\left(P_{i}\right)$ and progressively increasing standard deviation values for the different separation units. Table 32 shows the input parameters for this analysis, including the mean recovery value and the three levels of standard deviation used in the analysis. These values were integrated into the standard MCS and LPE procedures described above.

Figure 3-9 compares the relative uncertainty of circuit ore recovery using MCS and LPE at three levels of input variance. The values of coefficient of determination $(0.995,0.994$, and 0.988 for standard deviation of $10 \%, 20 \%$, and $30 \%$ of mean value, respectively) verify the capability of LPE-LCA tool; however, these results also show the deteriorating accuracy of the method as the level of variance increases. These result bolsters the argument by Xiao 
and Vien (2003); however, they should not necessarily preclude the benefits of the LPE approach. Even at a relatively high level of uncertainty (i.e. a standard deviation $30 \%$ of the mean for the third case), the results of the two methods coincide with a coefficient of determination of approximately 99\%. Not only this level of accuracy is suitable for most early stage design decisions, the likelihood of having such a high value of standard deviation is very low for most mineral processing systems.

Additionally, Figure 3-9 shows that there is little correlation between the type (or complexity) of the circuit design and the deviation between the MCS and LPE results. While the four-unit circuits (e.g. C33 and C34) do exhibit the highest deviation at the low level of input value standard deviation, other two-(e.g. C2) and three-unit (e.g. C10, C11, and C30) circuits exhibit similar deviation at the higher levels of input value variance. These observations indicate that the precision of LPE method is not necessarily a direct function of separation circuit complexity, but rather, the level of input standard deviations is the dominant factor in the approximation error raised by LPE.

Consequently, these results collectively indicate that the abbreviated LCA-LPE method is suitable for the initial evaluation of uncertainty in mineral processing circuit design, and the first derivative graphs, such as those presented in Figure 3-7, can be used to fundamentally show how uncertainty is propagating for different particle classes in different circuit designs.

\subsection{Summary and conclusions}

This paper has described a novel approach to circuit uncertainty quantification using circuit transfer functions derived from linear circuit analysis, and the law of the propagation of errors. The theoretical development of this method has been explained, and a Monte Carlo simulation was used to validate the applicability of the method. From this portion of the study, four key conclusions are derived:

- The separation circuit configuration has the ability to suppress or increase the uncertainty of various performance indicators relative to that of a single unit. The 
nature of this modification is dependent on both the stream interconnection and the stage recovery value.

- The law of propagation of errors, when combined with linear circuit analysis, is a suitable method to estimate the nature and direction of these modifications. This method only requires the stream connection diagram and estimates of the mean and uncertainty of input parameters, including stage recovery and feed grade, to provide these fundamental insights.

- While the LCA-LPE method can be simplified to only include the circuit configuration as the sole input parameter (see Equation 6), the most accurate formulation requires some knowledge of the mean recovery values and variance in each stage (see Equation 5). If replicate batch flotation data is available, this information can be used to estimate the relative standard deviation at a recovery value commensurate with the value expected in the industrial cell. This value can then be used to refine the analysis.

- The accuracy of the LCA-LPE method is dictated by the relative standard deviation of the input parameters, with more uncertainty in the input parameters producing less accurate predictions. Nevertheless, at reasonable levels of uncertainty, the LCALPE results coincide very well with the MCS, validating the utility of the method in preliminary circuit design.

Altogether, the methods described in this chapter provide another tool to the circuit designer who is working with limited input data early in the design process. In addition to the traditional technical objective functions (grade, recovery, separation efficiency), this method provides a quantitative basis to implement multi-objective function optimization, whereby the technical parameters are maximized, while risk or uncertainty is minimized.

\subsection{Bibliography}

Bárdossy G, Fodor J. Traditional and new ways to handle uncertainty in geology. Natural Resources Research. Springer; 2001;10(3):179-87.

Bosch M, Cara L, Rodrigues J, Navarro A, Diaz M. A Monte Carlo approach to the joint estimation of reservoir and elastic parameters from seismic amplitudes. Geophysics. 
Society of Exploration Geophysicists; 2007;72(6):O29-O39.

Caers J. Modeling uncertainty in earth sciences. Wiley, Chichester; 2011. p. 246.

Calisaya DA, López-Valdivieso A, Marcos H, Gálvez EE, Cisternas LA. A strategy for the identification of optimal flotation circuits. Minerals Engineering. Elsevier; 2016;96:157-67.

Cisternas LA, Jamett N, Gálvez ED. Approximate recovery values for each stage are sufficient to select the concentration circuit structures. Minerals Engineering. Elsevier; 2015;83:175-84.

Cisternas LA, Lucay F, Gálvez ED. Effect of the objective function in the design of concentration plants. Minerals Engineering. Elsevier; 2014;63:16-24.

Cisternas LA, Méndez DA, Gálvez ED, Jorquera RE. A MILP model for design of flotation circuits with bank/column and regrind/no regrind selection. International Journal of Mineral Processing. Elsevier; 2006;79(4):253-63.

Dimitrakopoulos R. Conditional simulation algorithms for modelling orebody uncertainty in open pit optimisation. International Journal of Surface Mining, Reclamation and Environment. Taylor \& Francis; 1998;12(4):173-9.

Ghaffari A, Hayati M, Shekholeslami A. Probability and Sensitivity Analysis in Flotation Circuit of Bama Lead and Zinc Processing Plant Using Monte Carlo Simulation Method. Mineral Processing and Extractive Metallurgy Review. Taylor \& Francis; 2012;33(6):416-26.

Guria C, Varma M, Mehrotra SP, Gupta SK. Simultaneous optimization of the performance of flotation circuits and their simplification using the jumping gene adaptations of genetic algorithm-II: More complex problems. International Journal of Mineral Processing. Elsevier; 2006;79(3):149-66.

Guria C, Verma M, Gupta SK, Mehrotra SP. Simultaneous optimization of the performance of flotation circuits and their simplification using the jumping gene adaptations of genetic algorithm. International Journal of Mineral Processing. Elsevier; 2005;77(3):165-85. 
Harris C, Cuadros-Paz A. Species interaction in flotation: a laboratory-scale semi-batch study. International Journal of Mineral Processing. Elsevier; 1978;5(3):267-83.

Hoffman FO, Hammonds JS. Propagation of uncertainty in risk assessments: the need to distinguish between uncertainty due to lack of knowledge and uncertainty due to variability. Risk analysis. Wiley Online Library; 1994;14(5):707-12.

Jamett N, Cisternas LA, Vielma JP. Solution strategies to the stochastic design of mineral flotation plants. Chemical Engineering Science. Elsevier; 2015;134:850-60.

Jamett N, Vielma JP, Cisternas LA. Design of flotation circuits including uncertainty and water efficiency. Symposium on Computer Aided Process Engineering. 2012. p. 20.

Karamoozian M, Shafaei S, Kakaie R, Noaparast M, Zeidabadi S, Ghasemi S. SAG mill power draw simulation using variance propagation approach. Iranian Journal of Science and Technology, Transaction B, Engineering. 2008;32(B6):619-29.

King RP. Modeling and simulation of mineral processing systems. Elsevier; 2012.

Der Kiureghian A, Ditlevsen O. Aleatory or epistemic? Does it matter? Structural Safety. Elsevier; 2009;31(2):105-12.

Kraslawski A. Review of applications of various types of uncertainty in chemical engineering. Chemical Engineering and Processing: Process Intensification. Elsevier; 1989;26(3):185-91.

Lauder D, McKee D. The impact of circulating loads on flotation circuit performance. 13th CMMI Congress. 1986. p. 41-7.

Lisitsin VA, Porwal A, McCuaig TC. Probabilistic fuzzy logic modeling: quantifying uncertainty of mineral prospectivity models using Monte Carlo simulations. Mathematical Geosciences. Springer; 2014;46(6):747-69.

Lucay F, Cisternas L, Gálvez E. Global sensitivity analysis for identifying critical process design decisions. Chemical Engineering Research and Design. Elsevier; 2015; 
Lucay F, Mellado ME, Cisternas LA, Gálvez ED. Sensitivity analysis of separation circuits. International Journal of Mineral Processing. Elsevier; 2012;110:30-45.

Luttrell G, Kohmuench J, Stanley F, Trump G. Improving spiral performance using circuit analysis. Minerals \& metallurgical processing. Society for Mining, Metallurgy and Exploration; 1998;15(4):16-21.

Lynch A, Johnson N, Manlapig E, Thorne C. Mineral and coal flotation circuits: Their simulation and control. Elsevier, Amsterdam; 1981.

McKeon T, Luttrell G, others. Optimization of multistage circuits for gravity concentration of heavy mineral sands. Minerals \& Metallurgical Processing Journal. SME; 2012;29(1):1-5.

McKeon T, Luttrell GH. Application of linear circuit analysis in gravity separator circuit design. 2005.

Meloy T. Analysis and optimization of mineral processing and coal-cleaning circuits-circuit analysis. International Journal of Mineral Processing. Elsevier; 1983;10(1):61-80.

Meloy TP, Clark N, Glista JJ. Effect of density variations in heavy-media rougher-cleanerscavenger cells-circuit analysis. International journal of mineral processing. Elsevier; 1986;16(3):169-78.

Mendez DA, Gálvez ED, Cisternas LA. State of the art in the conceptual design of flotation circuits. International Journal of Mineral Processing. Elsevier; 2009;90(1):1-15.

Merks J. Process simulation with spreadsheet software. Minerals \& metallurgical processing. Society for Mining, Metallurgy and Exploration; 1999;16(2):29-36.

Montenegro MR, Bruckard WJ, Gálvez ED, Cisternas LA. Arsenic-rejection flotation circuit design and selection based on a multiple-objective evaluation. Minerals Engineering. Elsevier; 2013 a;45:22-31.

Montenegro MR, Gálvez ED, Cisternas LA. The effects of stage recovery uncertainty in the performance of concentration circuits. International Journal of Mineral Processing. Elsevier; 2015;143:12-7. 
Montenegro MR, Sepúlveda FD, Gálvez ED, Cisternas LA. Methodology for process analysis and design with multiple objectives under uncertainty: Application to flotation circuits. International Journal of Mineral Processing. Elsevier; 2013 b;118:15-27.

Mooney CZ. Monte carlo simulation. Sage Publications; 1997.

Morgan MG, Henrion M, Small M. Uncertainty: a guide to dealing with uncertainty in quantitative risk and policy analysis. Cambridge University Press; 1992.

Noble A, Luttrell G. Value-based objective functions applied to circuit analysis. Minerals \& Metallurgical Processing. 2015;32(1).

Noble A, Luttrell GH. The matrix reduction algorithm for solving separation circuits. Minerals Engineering. Elsevier; 2014;64:97-108.

Noble CA. Analytical and Numerical Techniques for the Optimal Design of Mineral Separation Circuits. Virginia Tech; 2013;

Perry R. The impact of data correlation on the material balance problem. Minerals engineering. Elsevier; 1997;10(6):585-602.

Regan HM, Hope BK, Ferson S. Analysis and portrayal of uncertainty in a food-web exposure model. Human and Ecological Risk Assessment. Taylor \& Francis; 2002;8(7):1757-77.

Schena G, Villeneuve J, Noël Y. A method for a financially efficient design of cell-based flotation circuits. International journal of mineral processing. Elsevier; 1996;46(1):1-20.

Schena G, Zanin M, Chiarandini A. Procedures for the automatic design of flotation networks. International journal of mineral processing. Elsevier; 1997;52(2):137-60.

Sepúlveda FD, Cisternas LA, Gálvez ED. The use of global sensitivity analysis for improving processes: Applications to mineral processing. Computers \& Chemical Engineering. Elsevier; 2014;66:221-32.

Simonsen H, Perry J. Risk identification, assessment and management in the mining and metallurgical industries. Journal-South African Institute of Mining and Metallurgy. 
South African Institute of Mining and Metallurgy; 1999;99(6):321-8.

Thomopoulos NT. Essentials of Monte Carlo simulation: Statistical methods for building simulation models. Springer Science I\& Business Media; 2012.

Williams M, Fuerstenau D, Meloy T. Circuit analysis-general product equations for multifeed, multistage circuits containing variable selectivity functions. International Journal of Mineral Processing. Elsevier; 1986;17(1):99-111.

Williams M, Meloy T. Dynamic model of flotation cell banks-circuit analysis. International Journal of Mineral Processing. Elsevier; 1983;10(2):141-60.

Williams M, Meloy T. On the definition and separation of fundamental process functions. International Journal of Mineral Processing. Elsevier; 1989;26(1):65-72.

Wills BA. Wills' mineral processing technology: an introduction to the practical aspects of ore treatment and mineral recovery. Butterworth-Heinemann; 2011.

Xiao Z, Vien A. Limitations of variance analysis using propagation of variance. Minerals engineering. Elsevier; 2003;16(5):455-62.

Zio E. The Monte Carlo simulation method for system reliability and risk analysis. Springer; 2013. 


\title{
Chapter 4
}

\section{Application of Linear Circuit Analysis and Functional Unit Evaluation to the Sensitivity Analysis of Separation Circuits}

\author{
(ABSTRACT)
}

The estimation and analysis of uncertainty propagation in mineral processing circuits is an important, but challenging, aspect of a comprehensive circuit design strategies. Owing to the sophisticated and complex modeling requirements, many of the current circuit optimization tools rely on deterministic models, despite the ubiquity of uncertainty in the model input parameters. Likewise, many of the uncertainty assessment protocols rely on purely mathematical solutions that provide no insight on how particular circuit modifications will influence uncertainty propagation. As a supplement to these methods, the current chapter describes a method of assessing and evaluating circuit uncertainty by integrating the fundamentals of linear circuit analysis and law of propagation of error (according to chapter 3) as well as separation functional units' concept. This analysis shows how the addition or modification of units within circuits, based on functional unit definitions, can be used to predict how uncertainty propagation will change, even with limited a priori data. The methods are then employed the proposed analytical strategy, described in the previous chapter, to show which units are most influential in circuit uncertainty contribution, and lastly, the capabilities of this innovative approach are illustrated through simple multi-stage circuit configurations and then a modeled industrial copper processing plant. The new technique may offer considerable value by providing fundamental information on why specific circuits propagate uncertainty in drastically different ways.

Keywords: circuit design; uncertainty; sensitivity analysis; linear circuit analysis; law of propagation of error; functional unit. 


\subsection{Introduction}

\subsubsection{Background}

The analysis of uncertainty propagation in mineral processing separation circuits is an essential and significant, yet challenging, aspect of an optimal circuit design procedure. Conventional mineral separation circuits, particularly flotation circuits, often employ complex multi-unit configurations with numerous recirculating streams. The design and optimization of these circuit configurations is a challenging endeavor as the number of feasible and sub-optimal solutions is vast. Over the last 40 years, researchers have coupled heuristic methods with laboratory experiments to identify suitable circuit configurations, and most recently, strategies for numeric optimization of circuit configurations have been attempted using mathematical process models and advanced search algorithms. Extensive reviews of these design and optimization methods have been carried out in several studies, including Mendez et al. (2009) and Noble (2013). Unfortunately, the mathematical complexity of many of these optimization approaches often precludes the assessment of uncertain parameters, as even the deterministic models are extremely complex and difficult to solve.

As comprehensively explained in previous chapters, the minerals industry is subject to several sources of epistemic and stochastic uncertainty in both technical and economic factors, which namely include mineral price, ore grade, material separation characteristics, and operational variability. While some prior studies have investigated the influence of uncertainty on isolated unit operations or components of a mineral separation circuit, few studies have holistically assessed the influence of the separation circuit configuration on the nature and extent of uncertainty propagation. Examples of prior studies include those by Xiao and Vien (2003); Karamoozian et al. (2008); and Ghaffari et al. (2012), who have all used Monte Carlo techniques to assess the influence of uncertainty propagation in mineral processing circuits. More recently, studies by Montenegro et al. (2013) and Jamett et al. (2015) have demonstrated novel methods of integrating uncertain parameters in a more comprehensive and rigorous circuit optimization protocol. Prior chapters have provided a review of the most recent studies on the mineral processing circuit design and evaluation under uncertainty and then described a novel method using linear circuit analysis coupled with law of the propagation of errors (Amini and Noble, 2017). Building upon that work, 
the current chapter will assess the capability of the proposed methodology in analyzing the circuit uncertainty and identifying the influential parameters on this uncertainty propagation.

The collective results of these studies show that mineral processing circuits could benefit from a more rigorous approach to uncertainty assessment, but very few methods provide fundamental insight on the causal factors that influence uncertainty propagation. Furthermore, despite the adaptation and development of advanced instrumentation and control techniques, the minerals industry will perpetually require a better method of predicting and managing uncertainty, as complex ore mineralogy and diminishing ore grades will require more sophisticated, profitable, and efficient mineral processing circuit flowsheet and operations. Therefore, there is a critical need to develop comprehensive but straightforward strategies to not only find an optimal circuit solution under uncertainty but also properly evaluate existing circuit performance for possible improvements.

\subsubsection{Sensitivity analysis for separation circuits}

To adequately evaluate and optimize circuit performance, an efficient design strategy must not only predict the global circuit selectivity and uncertainty, but it must also identify which parameters are most influential in the circuit performance and thus merit further consideration. While not a true comprehensive uncertainty quantification approach, circuit sensitivity analysis provides a straightforward approach to evaluating uncertainty, whereby the results can be aggregated to develop trends and insights on which parameters are most influential in particular situations.

While recent authors have developed and refined the sensitivity analysis approach (e.g. Lucay et al., 2012), the earliest reference to a rigorous approach to circuit reduction was by Meloy (1983a, 1983b) using a method known as linear circuit analysis (LCA). LCA describes a mathematical procedure to derived analytical circuit solutions that define circuit recovery in terms of unit recovery values utilizing mass balance principles. By employing analytical circuit solutions, Meloy showed that LCA can be used to rank separation circuit designs based on circuit performance selectivity. The original idea was later developed into a series of publications examining various aspects and applications of the original methodology. Some examples are the analysis of dynamic flotation cell models (Williams and Meloy 1983), 
multi-feed multi-stage separators (Williams et al. 1986), and the sensitivity of dense media circuit performance to density variations (Meloy et al. 1986). Recently, Noble and Luttrell (2014) demonstrated a method to produce analytical circuit solutions using nodal analysis.

By utilizing the analytical circuit solution (also denoted the global recovery function), Lucay et al. (2012) later showed how sensitivity analysis can be used to rigorously identify influential units in complex circuits. After deriving the global recovery function, the partial derivative of this expression was calculated with respect to the recovery of the unit under scrutiny. Given the range of expected recovery values for the unit under scrutiny and consistent recovery values for all other separation units, the magnitude of this partial derivative was estimated and then plotted. Local minima and maxima in the plots were identified. This process was then repeated for all separation units, and the overall magnitude of each partial derivative was compared to determine the unit with the greatest influence on the circuit recovery selectivity. Since the behavior of each sensitivity plot may change with respect to the value of each parameter, the proposed local sensitivity analysis (LSA) methodology may fail to accurately identify globally influential parameters.

Given these drawbacks to the LSA approach, Sepúlveda et al. $(2013,2014)$ used various SA tools (e.g. Sobol' and Morris methods) to simultaneously investigate the significance of operational conditions in each separation unit for determining global recovery and product grade indicators. Sobol' methodology, as a variance-based global sensitivity analysis (GSA) tool, was initially utilized to decompose the total variance of the model to partial variances. Afterward, the value of each partial variances was calculated using Monte Carlo simulation. Subsequently, given the individual and interactional partial variances, the sensitivity of various separation performance indicators to each parameter was estimated. In addition to the Sobol' methodology, the authors have applied Morris method to determine the linear, non-linear, and interactional effects of each input parameter on the overall circuit selectivity and uncertainty propagation. The proposed GSA methodologies have an excellent capability for identifying influential parameters in the separation circuit performance. However, these purely mathematical solutions often require specialized software packages (e.g. SimLab and GUI-HDMR) and provide few fundamental insights on the types and nature of circuit designs that propagate uncertainty - information that would be highly beneficial to a circuit 
designer working on preliminary greenfield or operating design problems without exhaustive datasets.

\subsubsection{Objectives}

The objective of the current chapter is to present a complementary approach to quantitative circuit uncertainty assessment and sensitivity analysis using the concept of functional unit definitions originally provided by Williams and Meloy (1989). While this technique was originally used to solely characterize circuit selectivity, the current method expands this concept to also include circuit uncertainty propagation when used along with the analytical method (LCA-LPE approach) proposed in the prior chapter. The outcome is a set of simple heuristics that not only supplement more complex optimization protocols, but provide insight on why certain circuit configurations propagate uncertainty at different levels. In summary, the following sub-objectives are identified:

- Review the principles of LCA, LPE, and functional unit concepts and propose a methodology that combines all three elements (section 4.2);

- Evaluate the LCA, functional unit, and LPE strategies to analytically assess separation circuit performance and identify influential parameters in the circuit selectivity and uncertainty propagation (section 4.3 and 4.4);

- Demonstrate the proposed methodology in an application example using a modeled copper processing plant (section 4.5).

\subsection{Mathematical Basis}

\subsubsection{Review of LCA}

Prior chapter comprehensively described the fundamentals of linear circuit analysis approach. This section briefly reviews LCA to adequately explain the capability of this method for the circuit uncertainty analysis when it combines with the principles of law of propagation of error and functional unit concept. LCA refers to an analytical circuit analysis 


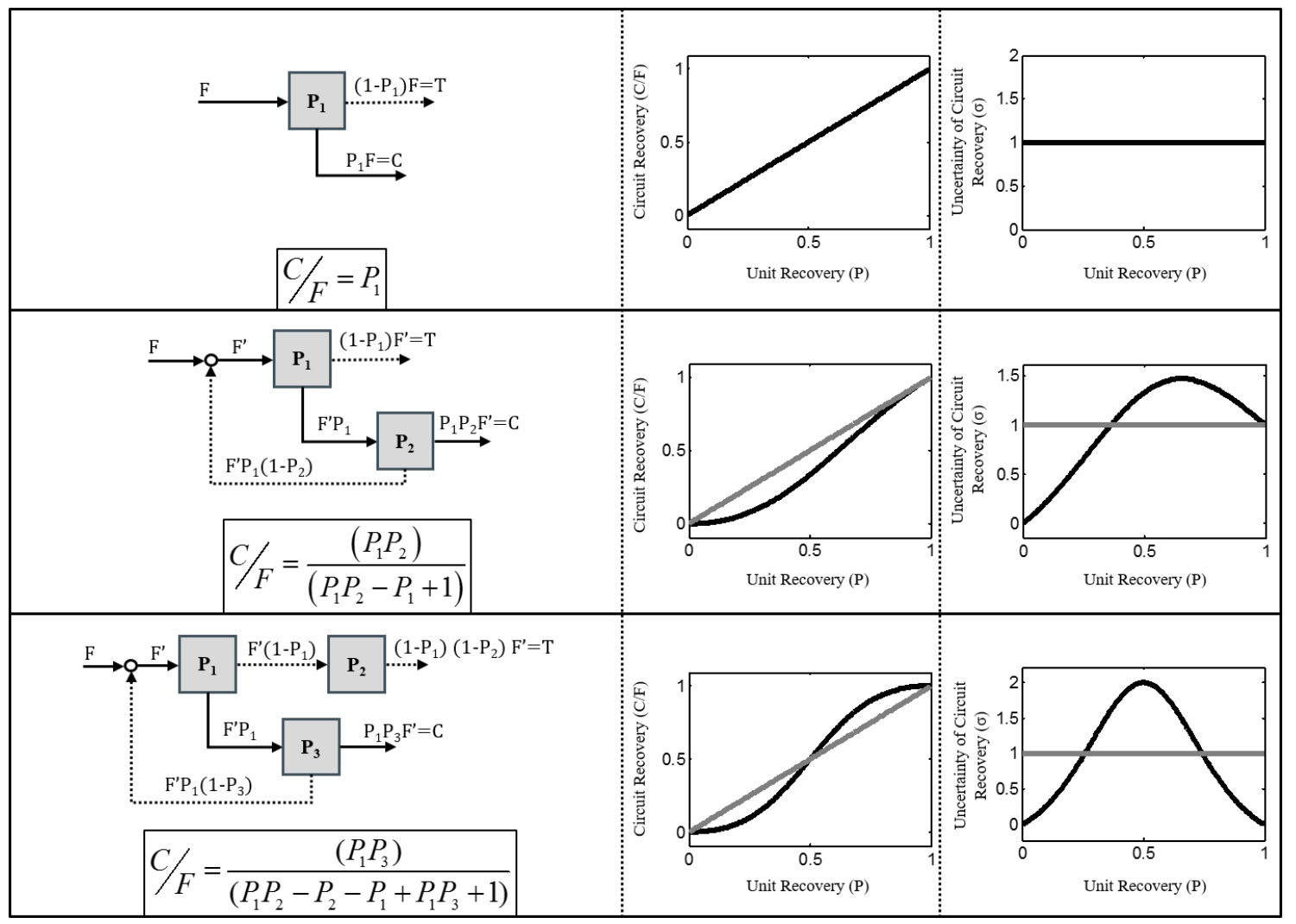

Figure 4-1: Analytical circuit transfer functions for various one, two, and three-unit separation circuit examples (left panel) along with the graphical representation of recovery

parameter (middle panel) and uncertainty recovery (right panel) as a function of unit recovery values.

approach that is employed to determine circuit separation performance solely based on separation unit arrangements and stream interconnections as well as separation probabilities (Meloy 1983a). Separation probabilities $\left(P_{i}\right)$, may be defined as the ratio of mass flow rate of concentrate stream $\left(C_{i}\right)$ over the mass flow rate of feed stream $\left(F_{i}\right)$ for the particular class of particles in question. The separation probability or transfer function, $P_{i}$, indicates the percentage of the specific class of particles in the unit feed that reports to the concentrate $\left(C_{i}\right.$ $\left.=P_{i} F_{i}\right)$ and tailing streams $\left(T_{i}=\left(1-P_{i}\right) F_{i}\right)$. Applying the principles of mass balance and separation probabilities results in the determination of the analytical circuit transfer function $\left(C_{i} / F_{i}\right)$ for any physical separation circuit without the need to specify any numerical values to unknown parameters including $P_{i}, F_{i}, C_{i}$, and $T_{i}$. The left panel of Figure 4-1 illustrates a series of simple separation circuit examples along with mass flow in each stream and final 
analytical circuit transfer functions. The analytical circuit transfer function formulation directly reflects the circuit recovery parameter for the specific class of particles as a function of units' separation probabilities:

$$
C / F=f\left(P_{1}, P_{2}, \ldots, P_{i}\right)
$$

The middle panel of Figure 4-1 shows the circuit recovery at different values of unit recovery when all $P$ values are considered equal. In this case, unit recovery values on the $\mathrm{x}$ axis can refer to a specific class of particles that are recovered at a specific rate (i.e., a $P$ value of 0.25 refers to a class of particles that have a $25 \%$ recovery in a single unit operation). As indicated in this figure, recovery of the two-unit circuit (rougher-cleaner) is lower than the single-unit case through all unit $P$ values. However, the recovery of the three-unit circuit design (rougher-scavenger-cleaner) is greater for high unit recovery values and lower for low values of unit recovery. This formulation can then be used with feed grade and other parameters to determine most circuit performance indicators such as circuit product grade, separation efficiency, and circuit yield.

\subsubsection{Review of LPE-LCA method}

As thoroughly discussed in the recent study by Amini and Noble (2017) and in chapter 3 , the analytical transfer function derived from the LCA approach can be used to quantify uncertainty propagation in mineral processing circuit designs utilizing the law of propagation of error (LPE) principle. In these cases, circuit robustness is defined as cases where small changes in unit recovery result in relatively small changes in overall circuit recovery. High uncertainty propagation is thus characterized by a lack of circuit robustness, as small changes in unit recovery will result in amplified changes to overall circuit recovery.

The combined LPE-LCA methodology may be employed to rank separation circuit designs based on the level of compounded uncertainty (i.e. lack of robustness) for various separation performance measures. In general, LPE predicts the uncertainty (i.e. standard deviation, $\sigma$ ) of a function based on the standard deviation of function input variables. As such, based on the principle of LPE, the uncertainty of the generic function of $F(X, Y, Z)$ can be determined, as follows: 


$$
\sigma_{F}=\sqrt{\left(\frac{\partial F}{\partial X}\right)^{2} \sigma_{X}^{2}+\left(\frac{\partial F}{\partial Y}\right)^{2} \sigma_{Y}^{2}+\left(\frac{\partial F}{\partial Z}\right)^{2} \sigma_{Z}^{2}}
$$

where $\sigma_{F}, \sigma_{X}, \sigma_{Y}$, and $\sigma_{Z}$ are the standard deviation of function $(\mathrm{F})$ and input variables $(\mathrm{X}, \mathrm{Y}, \mathrm{Z})$; and $\left(\frac{\partial F}{\partial X}\right),\left(\frac{\partial F}{\partial Y}\right)$, and $\left(\frac{\partial F}{\partial Z}\right)$ are the derivatives of the function $\mathrm{F}$ with respect to $\mathrm{X}, \mathrm{Y}$, and $\mathrm{Z}$.

Similarly, uncertainty propagation of separation circuits may be predicted as a function of standard deviation of input uncertain variables such as unit recovery and feed grade. To calculate the uncertainty of global recovery for specific particle classes, following formulation may be used:

$$
\sigma_{(C / F)}=\sqrt{\left(\frac{\partial(C / F)}{\partial P_{1}}\right)^{2} \sigma_{P_{1}}^{2}+\left(\frac{\partial(C / F)}{\partial P_{2}}\right)^{2} \sigma_{P_{2}}^{2}+\ldots+\left(\frac{\partial(C / F)}{\partial P_{N}}\right)^{2} \sigma_{P_{N}}^{2}}
$$

where $\mathrm{N}$ is the number of unit operations in the circuit. On the common assumption that all unit recovery $\left(P_{i}\right)$ values are equal, uncertainty in the circuit recovery can be estimated by LPE, and is given by:

$$
\sigma_{(C / F)}=\sqrt{\left(\frac{\partial(C / F)}{\partial P}\right)^{2} \sigma_{P}^{2}}
$$

This simplification reduces the problem to a single dimension, permitting simple graphical analysis. As shown in the right panel of Figure 4-1, after simplification, the level of uncertainty in circuit recovery factor can be solved analytically at different values of stage recovery, assuming all units have the same recovery value - the same assumption that was made to generate the middle panel. In this uncertainty plot, values greater than one indicate that the circuit uncertainty is intensified when compared to a single-unit case, while values less than one show that the uncertainty is mitigated. In practice, values less than one indicate 
that the circuit is more robust and less sensitive to small deviations in unit recovery - a value that can be correlated back to unique particle classes.

The single unit circuit (top panel) is trivial, as the circuit uncertainty remains constant through all $P$ values. However, as illustrated in the two lower panels, two-unit and three-unit circuits have the ability to suppress or intensify the level of uncertainty in circuit recovery when compared with the single-unit case. For example, both multi-unit circuits propagate lower level of uncertainty for low recovery particles $(P \leq 0.25)$, while the two-unit circuit demonstrates the high degree of uncertainty when treating mid-to-high recovery particles (e.g. $P \approx 0.65$ ). As demonstrated in prior publications, the principle of LCA and LPE can be extended for various separation performance measures including product grade and separation efficiency. Ultimately, the LPE-LCA approach may help circuit designers to design and evaluate separation circuits for cases of uncertainty in input parameters. Details on the calculation approach, applications, and implications of the LPE-LCA methodology are reported by Amini and Noble (2017). Others researchers have also demonstrated similar outcomes (Xiao and Vien 2003; Karamoozian et al. 2008).

\subsubsection{Review of process functional units}

As an extension of their initial work in linear circuit analysis, Williams and Meloy (1989) identified a simplified approach for the qualitative circuit evaluation using precisely defined process functional units. While the terms "rougher," "scavenger," and "cleaner" are used colloquially to define certain parts of a mineral processing circuit, Williams and Meloy (1989) refined these terms by providing rigorous definitions. In their work, they define: (1) rougher units as those that are fed by the circuit feed, (2) scavenger units as those that are fed by any tailings stream, and (3) cleaner units as those that are fed by any product stream. Given this nomenclature, a single unit operation in a typical circuit may fulfill one, two, or even all fundamental process functions simultaneously, thus creating seven unique combinations. Table 4-1 lists these seven functional units, their symbols in this manuscript, and their basic definitions. Additionally, Figure 4-2 illustrates some of the functional units in a multi-stage separation circuit.

Williams and Meloy (1989) used this concept of functional units to show how the best circuits employ units that only fulfill a single process function at a time (i.e. they minimize 
Table 4-1: Seven possible separation functional units in mineral processing circuits along with their symbols and input streams.

\begin{tabular}{|l|l|l|}
\hline Functional units & Symbol & Input \\
\hline Rougher & R & Circuit feed \\
\hline Cleaner & C & Product stream \\
\hline Scavenger & S & Tailings stream \\
\hline Rougher-Cleaner & RC & Circuit feed \& product streams \\
\hline Rougher-Scavenger & RS & Circuit feed \& tailings streams \\
\hline Rougher-Cleaner-Scavenger & RCS & Circuit feed \& product \& tailings streams \\
\hline Cleaner-Scavenger & CS & Product \& tailings streams \\
\hline
\end{tabular}

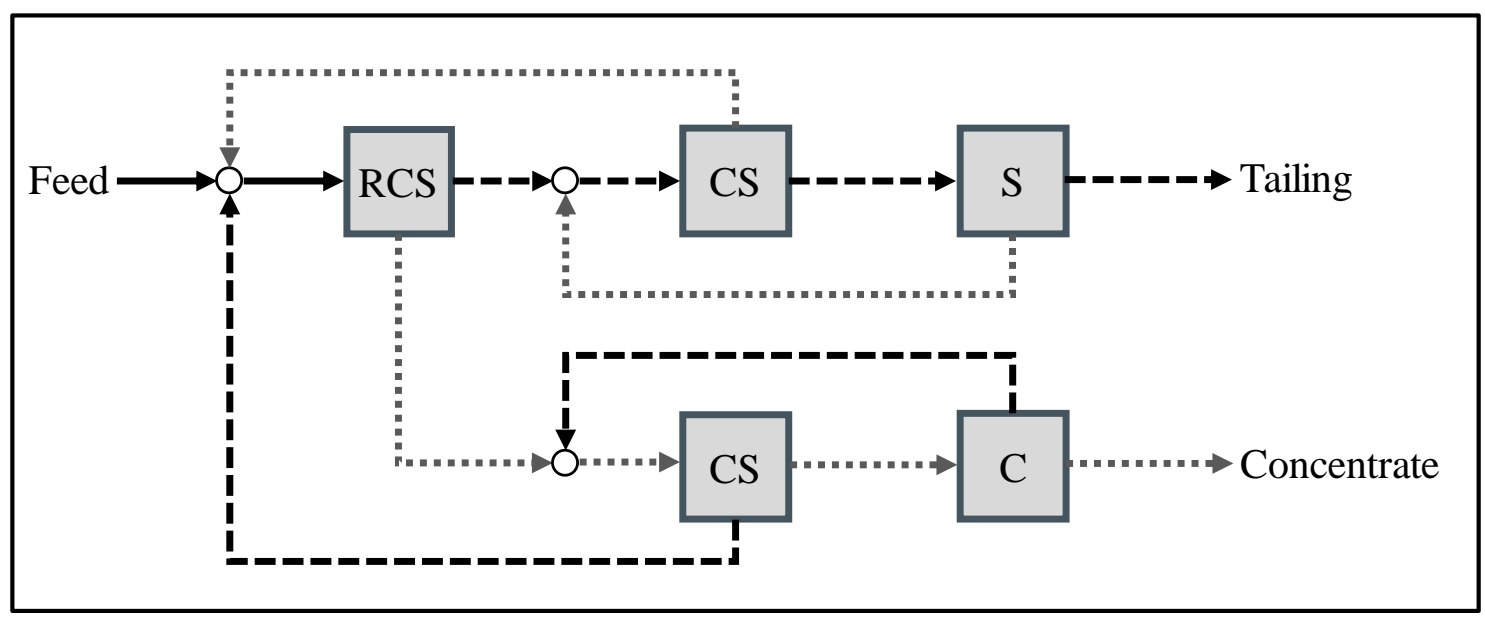

Figure 4-2: Illustration of several functional units in mineral processing circuit configuration.

the number of multi-function unit operations). This approach typically reduces the mass flow rate to individual units, and provides for simpler process benchmarking and control. They demonstrated this outcome by analyzing a series of circuits with identical circuit transfer functions $(\mathrm{C} / \mathrm{F})$, but with different functional unit utilization. They conclude that circuits composed of units with a single process function may require more units, but they enhance selectivity and control because of the decrease in mass flow feeding individual units. 
This fundamental concept may help circuit designers to adequately investigate the impact of possible circuit modifications on the various circuit performance measures (i.e. final product grade and global recovery) and possible changes in the stream mass flow rate.

\subsection{Methodology and data analysis}

Given the unique information provided by the functional unit descriptions, this paper seeks to evaluate the influence that functional unit utilization has on circuit uncertainty propagation, namely the information provided by the LPE-LCA approach. Furthermore, the LPE-LCA methodology is employed as a global sensitivity analysis tool to accurately identify the role of sources of uncertainty on the level of circuit selectivity and uncertainty propagation. When taken together, these analyses will not only show sources and magnitude of circuit uncertainty, but the functional unit concept will provide simple rules that a circuit designer can utilize. Together with the LPE-LCA methodology, this approach may provide a straightforward and comprehensive strategy for designing and retrofitting of greenfield and operating plants.

\subsubsection{Sensitivity analysis using principles of functional units}

To demonstrate the practical application of the LPE-LCA method for evaluating uncertainty propagation in mineral processing circuits, the relative uncertainty of the circuit recovery factor was first estimated and analytically illustrated for 31 generic one, two, and three-unit circuits. The graphical representation of these mineral processing circuits along with their analytical circuit transfer functions are shown in Figure 4-3. As described in the previous chapter, these 31 circuit configurations cover the majority of the standard design templates for one, two, and three-unit separation circuits and have been used by the authors in prior studies to demonstrate the robustness of the various LCA approaches (Noble and Luttrell 2014, 2015). As discussed in the mathematical basis section, the relative uncertainty (as calculated by Eq. 16) is determined as the ratio of circuit recovery standard deviation to the same value calculated for the single unit reference case. In this phase of the study, relative uncertainty was determined based on the assumption underlining equality of unit $P$ values (i.e. unit recovery) to simplify the analysis. Although this assumption may not be wholly valid for processing plants, it provides value in qualitatively analyzing circuit configurations under uncertainty, as it reduces the problem to a single dimension that can be visualized and 


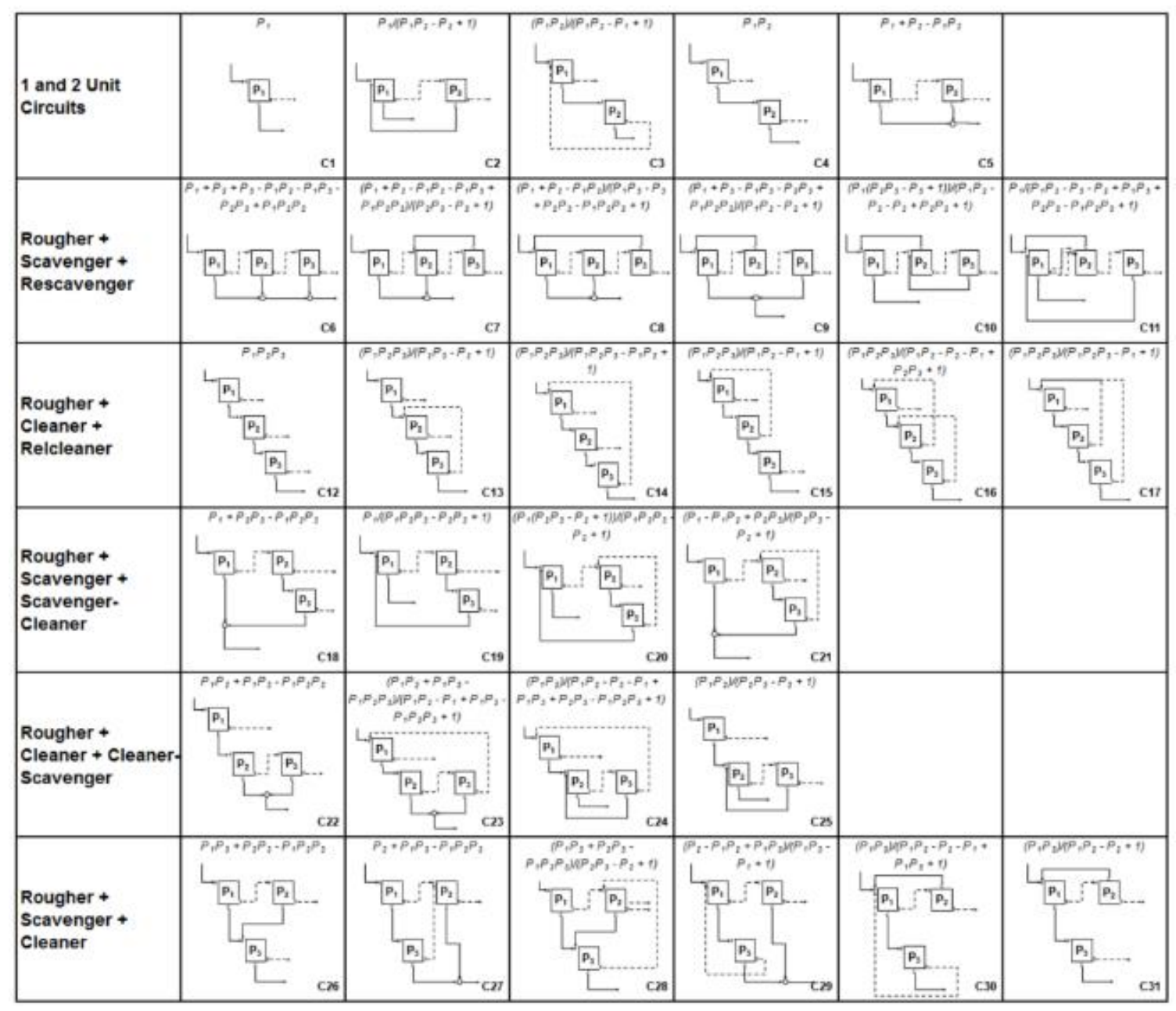

Figure 4-3: Graphical representation of typical one, two, and three-unit separation circuits.

assessed using standard tools. This approach provides a basis for deriving generic design rules; however, the approach can be readily applied at higher dimensions for problems with unequal unit $P$ values.

In addition to the LPE-LCA valuation, unit operations in each circuit were characterized using the seven combinations of basic functional units described in Table 4-1. By assessing the LPE-LCA valuations in this matter, patterns are identified in how certain functional units affect error propagation. Proper implementation of these basics may help circuit designers during the initial and modification design applications to study the impact of various functional units on the overall uncertainty propagation. 


\subsubsection{Sensitivity analysis using LPE-LCA approach}

In the study described in this chapter, LPE-LCA method was employed as a global sensitivity analysis technique to evaluate both uncertainty propagation and the relative magnitude of the various sources of uncertain in the 31 standard circuit templates. As discussed in section 4.2 (Eq. 15), LPE can be used to determine the uncertainty in circuit recovery as the function of input variables' partial derivatives and standard deviations. Eq. 15 may be further utilized to identify the role of input parameters (i.e. unit recovery as the source of uncertainty) in the final compounded uncertainty in circuit recovery. Therefore, Eq. 15 can be rewritten to estimate the contribution of various input factors to the variance of global recovery parameter, given by:

$$
\begin{aligned}
& \operatorname{Var}_{i}=\left(\frac{\partial(C / F)}{\partial P_{i}}\right)^{2} \sigma_{P_{i}}^{2} . \\
& \operatorname{Var}_{(C / F)}=\sigma_{(C / F)}^{2}=\sum_{i}^{n} \operatorname{Var}_{i}
\end{aligned}
$$

where $\operatorname{Var}_{i}$ is the contribution of ith unit recovery to the circuit recovery variance, $\operatorname{Var}_{(C / F)}$. As a result, the contribution index for various sources of uncertainty/error can be calculated from the components of variance in the LPE formulation, as follows:

$$
\text { Contribution Index }(\%)=\frac{\operatorname{Var}_{i}}{\operatorname{Var}_{(C / F)}} \times 100
$$

Given numeric values for unit recovery $\left(P_{i}\right)$ and its uncertainty $\left(\sigma_{P_{i}}\right)$, Eq. 18 can be used to determine the contribution of each function component on the circuit recovery variability. A similar procedure has been adopted and demonstrated by Napier-Munn (2014) for various mineral processing examples. To demonstrate the application of this concept, contribution indices of individual separation units were estimated for 31 circuit configurations through four intervals of unit recovery values (i.e. 10\%-30\%, 30\%-50\%, $50 \%-70 \%$, and $70 \%-90 \%)$. Each of these unit recovery intervals may be considered for a 
distinct class of particles. To simultaneously determine the contribution indices for various separation units, 100 calculation iterations were executed for each range covering all possible combinations of recovery values for individual separation unit operations. For simplicity, the values for standard deviations of unit recovery parameter were initially considered as $10 \%$ of the mean recovery values.

The majority of common global sensitivity analysis strategies are often complicated mathematical techniques and may require many model iterations. Conversely, LPE-LCA method can be employed as an effective variance-based mathematical model to correctly determine the contribution of static and uncertain input factors on the final propagated uncertainty in separation circuits. This methodology may help circuit designers to first identify the influential parameters on the circuit selectivity and uncertainty propagation, and then observe the importance of each source at different levels of standard deviations.

Upon the completion of this phase of the study, the proposed technique was applied in a modeled industrial example to properly demonstrate the suitability of LPE-LCA approach in determining the sensitivity of various circuit performance indicators, including copper and gangue recovery as well as product grade to different input factors.

\subsection{Results and Discussions}

\subsubsection{Sensitivity analysis using principles of functional units}

\section{Approach to the Analysis}

Figure 4-4 shows the relative uncertainty of circuit recovery (the result of Eq. 16) solved for all 31 circuit design templates (Figure 4-3) as a function of unit recovery values ranging continuously from 0 to 1 . This result was originally presented in Amini and Noble (2017) and is included here to demonstrate how those results can be extended and explained by incorporating other analytical tools, namely functional unit descriptions. The results in Figure 4-4 indicate that different circuit designs may display different behaviors under similar uncertain conditions. Furthermore, the data illustrate distinct levels of uncertainty propagation for various circuit configurations at $P$ values between 0 and 1 . 

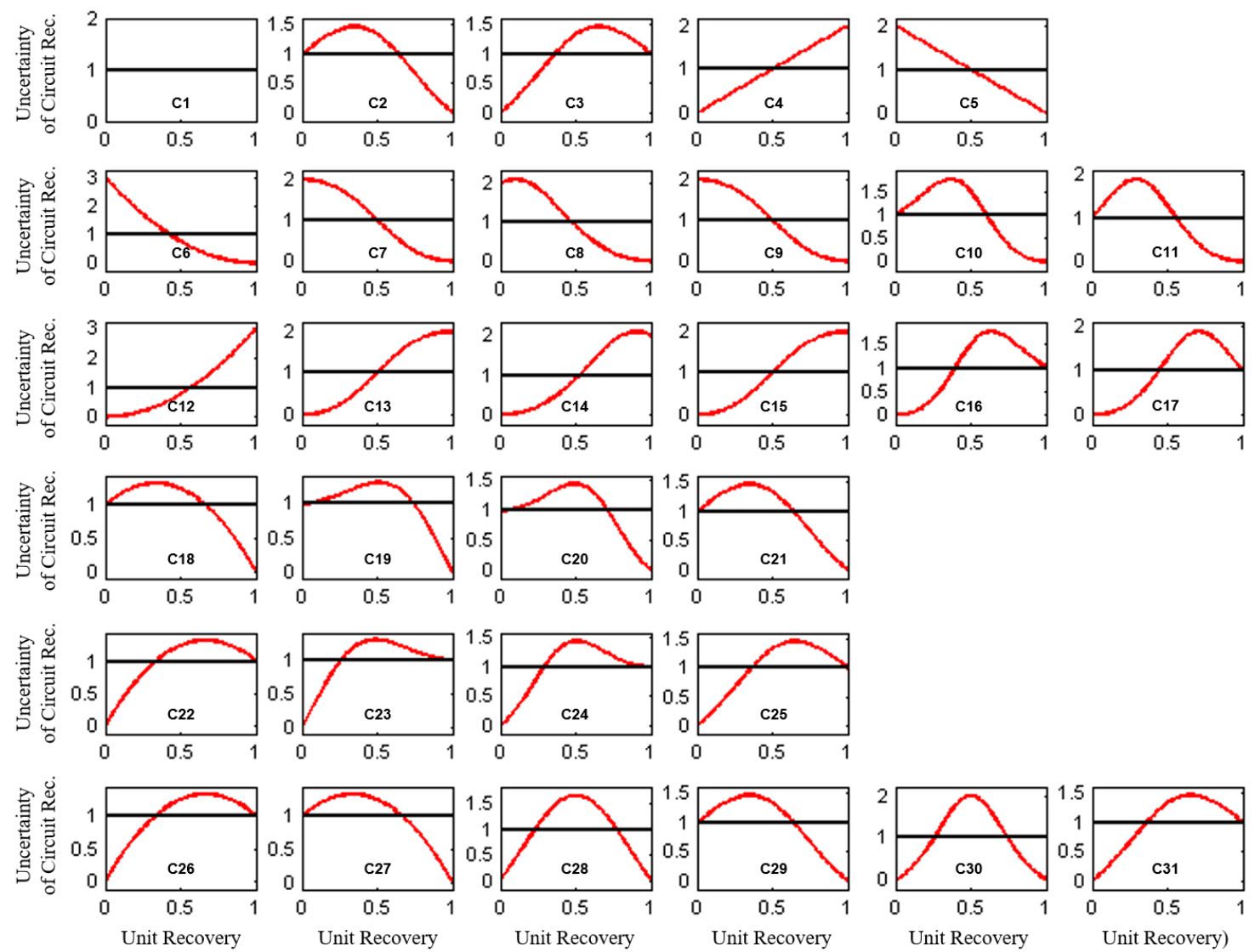

Figure 4-4: Uncertainty quantification for the generic one, two, and three-unit circuit configurations.

By design, the selection of the specific 31 circuit templates has included multiple instances for each of the seven unique functional unit combinations. To investigate the impact that these functional units have on circuit uncertainty propagation, the value of the relative circuit uncertainty propagation (i.e. Eq. 16) was determined and compared between circuits that utilize different combinations of functional units. To provide a basis for the comparison, the analysis was approached from two perspectives. First, the 31 circuits were assessed to identify pairs of circuits that contain a "base" circuit and a "modified" circuit where a single-function unit was added to the circuit. For example, $\mathrm{C} 1$ is the base single-unit circuit, and $\mathrm{C} 5$ is a modified version of $\mathrm{C} 1$ that has added a scavenger functional unit (Figure 4-3). All of these circuit pairs are identified in Table 4-2. As a second analysis, the 31 circuit pairs were assessed to identity pairs of circuits where a single-function unit was replaced with 
Table 4-2: Examples of circuit numerical comparisons for evaluation of scavenger and cleaner functional units' role in circuit uncertainty propagation.

\begin{tabular}{|c|c|c|c|c|}
\hline \multicolumn{5}{|c|}{ Unit Addition } \\
\hline Functional unit & Base Circuit & Modified Circuit & Impact on P=10\% & Impact on P=90\% \\
\hline \multirow{4}{*}{ Scavenger } & C1 & C5 & +0.80 & -0.80 \\
& C5 & C6 & +0.63 & -0.17 \\
& C2 & C9 & +0.77 & -0.20 \\
& C3 & C29 & +0.97 & -0.97 \\
& C4 & C22 & +0.17 & -0.63 \\
\hline \multirow{5}{*}{ Cleaner } & C1 & C4 & -0.80 & +0.80 \\
& C4 & C12 & -0.17 & +0.63 \\
& C3 & C15 & -0.20 & +0.77 \\
& C2 & C31 & -0.97 & +0.97 \\
& C5 & C18 & -0.63 & +0.17 \\
\hline
\end{tabular}

a multi-function unit. For example, C4 is the base case rougher-cleaner open circuit, and C3 is a modified version where the rougher functional unit has been replaced by a rougherscavenger unit via recirculation of the cleaner tailings. These circuit pairs are summarized in Table 4-3.

While the comparisons between circuit pairs can be made over a continuous range of recovery values using the curves in Figure 4-4, the analysis here has been simplified to only assess uncertainty propagation for high and low recovery particles, i.e. $\mathrm{P}=90 \%$ and $\mathrm{P}=10 \%$, respectively. These $\mathrm{P}$ values serve as rough surrogates for ore and gangue particles in typical mineral separation circuits, and this simplification clearly shows the trends that accompany specific functional units. The numeric values for the change in relative circuit uncertainty are presented with the specific circuit pairs in Table 4-2 and Table 4-3. Additionally, the numerical data in these tables are color-coded to readily distinguish the impact of each functional unit on the level of recovery uncertainty propagation (negative numbers are green; positive values are red; and values equal or close to zero are black). In the remaining part of this section, data presented in Table 4-2 and 4-3 are discussed in more detail and general conclusions are derived from these observations. 
Table 4-3: Examples of circuit comparisons for evaluation of scavenging and cleaning functionality influence on circuit uncertainty propagation.

\begin{tabular}{|c|c|c|c|c|}
\hline \multicolumn{5}{|l|}{ Unit Substitution } \\
\hline Functional unit & Base Circuit & Modified Circuit & Impact on $\mathrm{P}=10 \%$ & Impact on $\mathrm{P}=90 \%$ \\
\hline \multirow{6}{*}{ Rougher-Scavenger } & $\mathrm{C} 4$ & $\mathrm{C} 3$ & 0.03 & -0.60 \\
\hline & $\mathrm{C} 12$ & $\mathrm{C} 14$ & 0.00 & -0.33 \\
\hline & $\mathrm{C} 12$ & $\mathrm{C} 15$ & 0.00 & -0.46 \\
\hline & $\mathrm{C} 13$ & C16 & 0.01 & -0.74 \\
\hline & $\mathrm{C} 22$ & $\mathrm{C} 23$ & 0.05 & -0.14 \\
\hline & $\mathrm{C} 25$ & $\mathrm{C} 24$ & 0.03 & -0.17 \\
\hline \multirow{6}{*}{ Rougher-Cleaner } & $\mathrm{C} 5$ & $\mathrm{C} 2$ & -0.60 & 0.03 \\
\hline & C6 & $\mathrm{C} 8$ & -0.33 & 0.00 \\
\hline & C6 & C9 & -0.46 & 0.00 \\
\hline & $\mathrm{C} 7$ & C10 & -0.74 & 0.01 \\
\hline & C18 & C19 & -0.14 & 0.05 \\
\hline & $\mathrm{C} 21$ & $\mathrm{C} 20$ & -0.17 & 0.03 \\
\hline \multirow{4}{*}{ Scavenger-Cleaner } & C6 & C7 & -0.46 & 0.00 \\
\hline & C9 & $\mathrm{C} 10$ & -0.74 & 0.01 \\
\hline & $\mathrm{C} 12$ & $\mathrm{C} 13$ & 0.00 & -0.46 \\
\hline & $\mathrm{C} 15$ & $\mathrm{C} 16$ & 0.00 & -0.74 \\
\hline \multirow{3}{*}{ Rougher-Scavenger-Cleaner } & C31 & C30 & 0.04 & -0.93 \\
\hline & C29 & C30 & -0.93 & 0.04 \\
\hline & $\mathrm{C} 1$ & C30 & -0.73 & -0.73 \\
\hline
\end{tabular}

\section{Comparison of circuits with added units}

The numerical comparisons shown in Table 4-2 demonstrate that adding a scavenger functional unit anywhere in the circuit (i.e. in either the cleaning or scavenging legs) decreases the uncertainty propagation for the higher recovery particle classes $(P=90 \%)$ while increasing the recovery uncertainty for the lower $P$ value of $10 \%$. Furthermore, the addition of the scavenger unit, when it is the only scavenger in the circuit (for example, C5, C22, and C29), dramatically decreases the uncertainty propagation for higher $P$ values. For example, a 
particle with $90 \%$ recovery has a normalized uncertainty value of 1.0 in $\mathrm{C} 1$ against 0.2 in C5 (change of -0.8), and 1.19 in C3 against 0.23 in C29 (change of -0.97). Furthermore, these data indicate that adding scavenger functional unit in the cleaning leg may have a minor positive impact on the level of uncertainty propagation for the lower $P$ values. For example, at $10 \%$ recovery, C4 has a relative uncertainty value of 0.20 , while C22 (the same circuit with an added scavenger within cleaning leg) has an uncertainty value of $0.37(+0.17)$.

As expected, the addition of a cleaner functional unit on either the scavenging or cleaning legs indicates the exact opposite trends. As shown in Table 4-2, the relative uncertainty assessment for various circuit pairs that have an added cleaner demonstrates that cleaner functional units intensify the level of recovery uncertainty for the higher recovery particle class while reducing the relative uncertainty for low recovery particle classes.

\section{Comparison of circuits with modified units}

The same analysis can be used to assess the significance of multi-function units by carefully analyzing the circuits employing rougher-cleaner-scavenger (triple function unit) as well as cleaner-scavenger, rougher-cleaner, and rougher-scavenger functional units (dual function units). Similar to the previous discussion, the relative uncertainty of global recovery for high and low recovery values in various generic design examples were evaluated and compared (Table 4-3). In general, the data in Table 4-3 shows that elevating the functionality of separation units through the proper addition of recirculating loads can dramatically decrease the level of global recovery uncertainty, thereby boosting the robustness of separation circuit designs.

As illustrated in Table 4-3 and Figure 4-4, the replacement of rougher by rougherscavenger functional unit (i.e. reporting tailings streams into the rougher stage) often causes a significant decrease in the level of circuit uncertainty propagation for the recovery of particle classes with higher $P$ values. However, unlike the scavenger-only addition (first row of Table 4-2), the rougher-scavenger modification often does not influence the uncertainty propagation of the low recovery particles. A similar, but contradictory trend is observed for the rougher-cleaner substitution. 
Unlike the rougher-scavenger and rougher-cleaner modifications which always follow definitive trends, the scavenger-cleaner modification follows divergent trends depending on the nature of the modification. As shown in Table 4-3, the change in circuit uncertainty propagation in the C6-C7 and C9-C10 modifications follow the trends like that of the rougher-cleaner modification, while $\mathrm{C} 12-\mathrm{C} 13$ and $\mathrm{C} 15-\mathrm{C} 16$ follow that of the rougherscavenger. Both the C6-C7 and C9-C10 modification occur on a scavenging leg, where a pure scavenging unit is replaced with a scavenger-cleaner. Overall, this modification nets additional cleaner functional units, and thus the final result is a trend which appears similar to the rougher-cleaner results (i.e. a reduction in the uncertainty of low recovery particles and no change in high recovery particles). This same explanation also supports the $\mathrm{C} 12-\mathrm{C} 13$ and C15-C16 observations, albeit in the reverse trend.

Lastly, the rougher-scavenger-cleaner substitutions largely confirm the prior trends. For example, in comparing C31 to C30, the rougher-cleaner unit has been replaced by a roughercleaner-scavenger unit. The net result is added scavenging capacity, and as such, the trends follow that of the rougher-scavenger data (i.e. reduced uncertainty of high recovery particles and no net change of low recovery particles. The C29-C30 modification produces the opposite result, as the net result of that circuit is added cleaning capacity. Interestingly, in comparing C30 to the base case, C1, both cleaning and scavenging capacity has been added, and as such, the uncertainty in both high and low recovery particles is reduced.

\section{Design rules pertaining to uncertainty propagation}

Given the various observations presented above, the following generic circuit design rules may be derived. Generally, these rules indicate what types of uncertainty propagation outcomes should be expected after implementing a modification to the original circuit design:

- Adding pure scavenging capacity (without recycle) to an existing circuit will tend to reduce the uncertainty of high recovery particles, while increasing the uncertainty of low recovery particles. Adding pure cleaning capacity (without recycle) produce an identical, albeit reversed, trend. 
- Adding scavenging capacity via recirculation (e.g. transforming the rougher to a rougher-scavenger by recirculating the cleaner tailings) will tend to reduce the uncertainty of high recovery particle, while mitigating or eliminating the increase of uncertainty propagation in low recovery particles. Once again, the trend is reversed for added cleaning capacity.

- In more complex circuits, the net effect of uncertainty propagation in both high and low recovery particles can be predicted by assessing the net change in scavenging and/or cleaning capacity and then applying the two rules described above.

- Typically, the proper implementation of separation functional units with dual or triple functionality will considerably improve the robustness of the circuit design through suppressing the overall level of circuit uncertainty propagation.

\subsubsection{Results of global sensitivity analysis}

Figure 4-5 demonstrates the contribution indices (Eq. 18) of distinct functional units in 31 circuit design templates at various ranges of unit recovery values. In this plot, the data series have been designated (color, symbol, and line dash) to reflect the number of functions each unit is fulfilling. As explained in the mathematical basis section, these ranges of unit recovery values may illustrate the recovery $(P)$ values for different particle classes. Moreover, mathematical estimation of this index through the continuous recovery intervals accurately exhibit the trend associated with the influence of separation units on the circuit selectivity and variability through different values of recovery from 0 to 1 . The greater the value of contribution index for a unit operation means the higher the sensitivity of circuit recovery parameter to that unit separation performance. It is important to note that the "all $\mathrm{P}$ equal assumption" that was used in the prior analysis is not needed to interpret this data. Instead, these plots were produced assuming unique $\mathrm{P}$ values for each unit operation within a $20 \%$ recovery interval (e.g. 70\%-90\%), and the multiple curves on each plot can be read independently. 

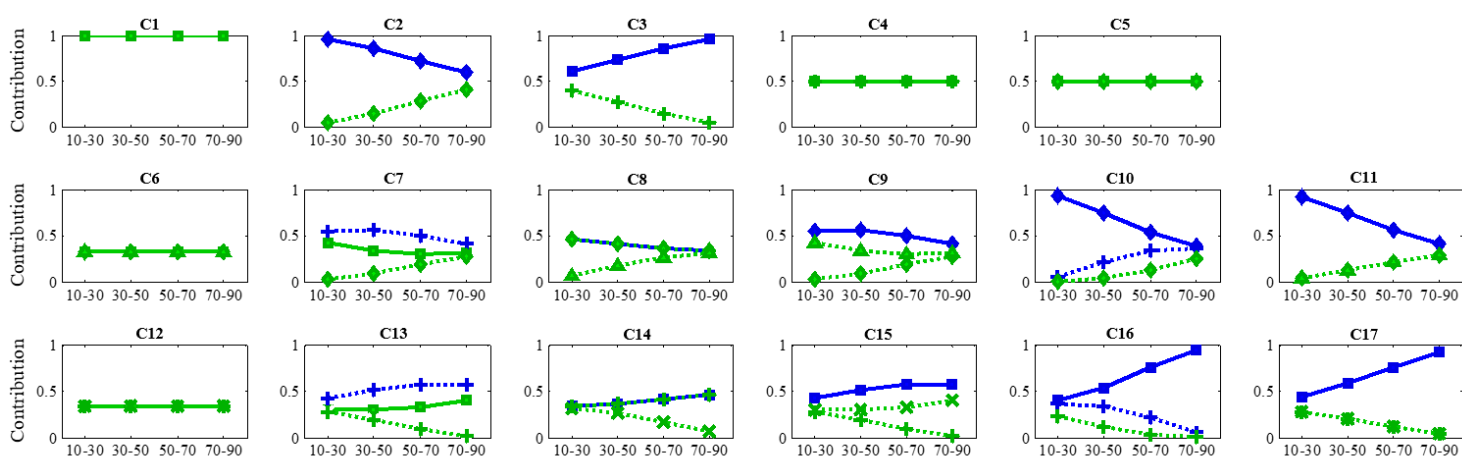

C13

C14

C15
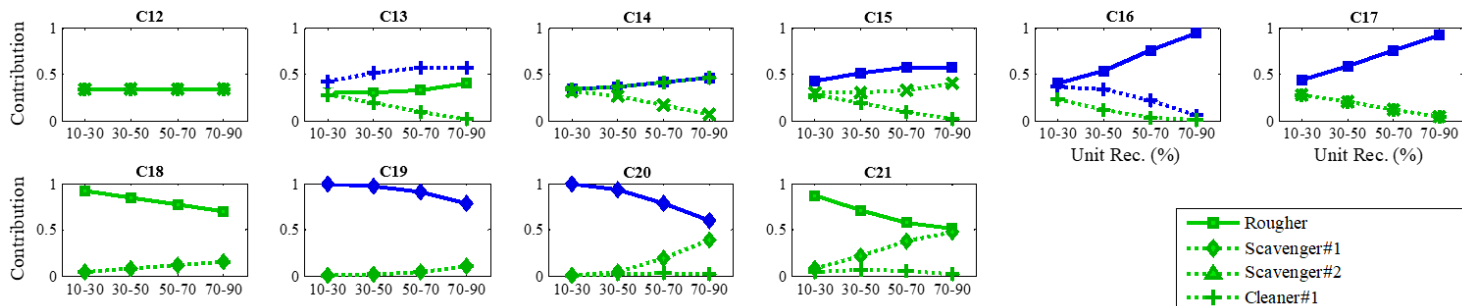

Unit Rec. (\%)

Unit Rec. (\%)
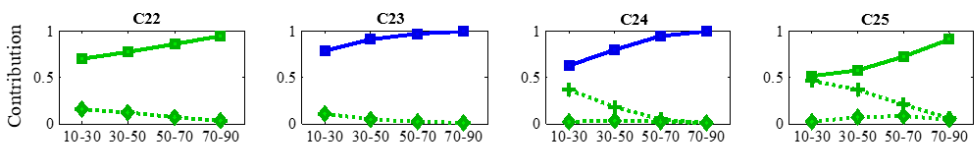

-. - Rougher - . - . - Scavenger \# - : + - . Cleaner\#1 - " $X$ - " Cleaner \#2 $\rightarrow$ Rougher-Scavenger $\rightarrow$ Rougher-Cleaner -.+.- Scavenger-Cleaner $\rightarrow$ Rougher-Cleaner-Scavenger
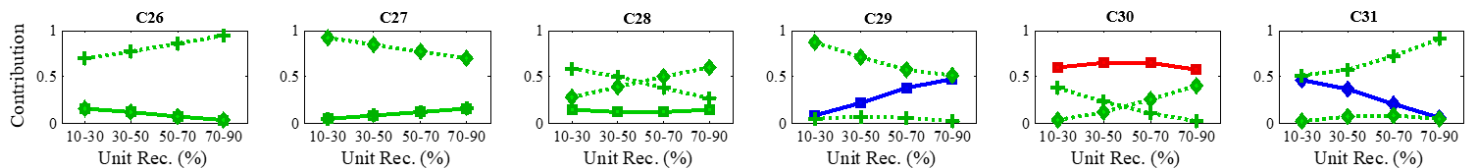

Figure 4-5: Contribution indices of separation units with the similar level of standard deviations.

Figure 4-5 shows the crucial importance of recirculating loads in identifying the most influential functional units on circuit uncertainty propagation. For instance, the rougherscavenger-cleaner functional unit in $\mathrm{C} 30$, which receives concentrate and tailings stream from both cleaning and scavenging legs, shows the highest level of contribution index. Replacement of this unit by rougher-cleaner or rougher-scavenger (see C29 and C31) results in declining the importance of roughering stage in the determination of compounded circuit uncertainty. Additionally, meaningful comparisons between circuit configurations indicate that rougher-cleaner and rougher-scavenger functional units demonstrate the greater values of contribution index when it compares with the contribution index of the rougher functional unit in the similar circuit design templates. While rougher-cleaner units have a significant role in determining the level of recovery uncertainty for particles classes with lower recovery values, the rougher-scavenger functional unit is considerably important through greater recovery values. A similar observation may be made when the behavior of scavenger- 
cleaner functional unit is compared against the behavior of cleaner or scavenger functional unit in the relatively similar circuit designs.

Interestingly, in Meloy's initial LCA paper (Meloy 1983a), he shows that the rougher unit is the most sensitive unit in deciding overall circuit separation efficiency, particularly in the balanced rougher-scavenger-cleaner circuit (C30). While the current contribution index analysis does not necessarily refute that claim, it does show several cases where the unit with a rougher function is not the most influential unit with respect to circuit recovery. For example, the circuit recovery of C7, C13, C26, C27, C28, C29, C31 is most influenced by a unit other than rougher as indicated by the greatest contribution index values. This result should prompt further scrutiny on Meloy's claim. In any event, circuit designers are cautioned against generalizing and extrapolating Meloy's claim to imply that the rougher unit is universally the most important in all cases and with respect to all performance metrics.

When the contribution index data is taken together with the conclusions of the prior section, this data demonstrates a unique outcome. The use of multi-function units tends to decrease the overall circuit uncertainty; however, it also tends to concentrate the contribution of that uncertainty in those high-function units. This information will help circuit designers to identify which portions of the circuit merit the most experimental or pilot work to reduce the design uncertainty.

\subsection{Application}

\subsubsection{Approach}

To further demonstrate the application of LPE-LCA methodology to sensitivity analysis for mineral processing circuits, an industrial copper processing circuit was studied considering uncertain stage recovery and feed grade factors. The circuit used in this example was first presented by Sepúlveda et al. (2014) who employed Morris sensitivity analysis technique to evaluate and improve the separation performance in a copper plant which was initially introduced by Hay and Martin (2004). By using the same circuit in the current analysis, the conclusions and outcomes between the three studies can be readily compared. In the current manuscript, the similar circuit configuration is first introduced (Figure 4-6a) 


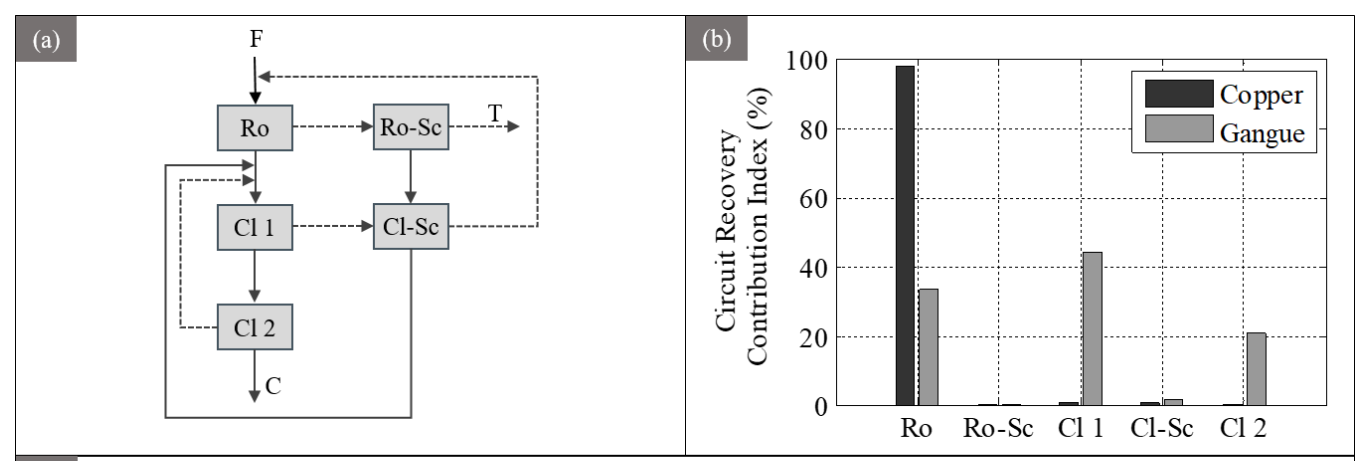

(c)

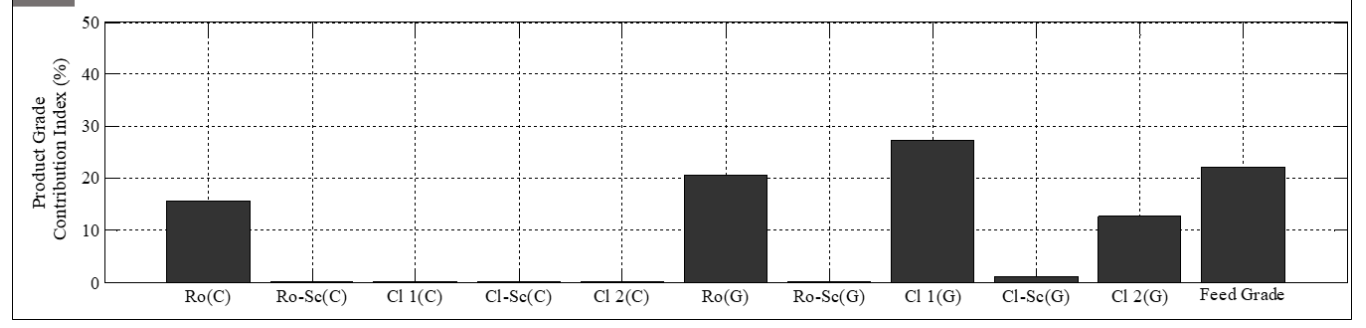

Figure 4-6: Original copper circuit configuration (a) along with separation unit contribution indices for copper and gangue recovery factors (b) and concentrate grade

and then thoroughly evaluated using LPE-LCA tool, as a global sensitivity analysis methodology.

As illustrated in Figure 4-6a, the copper separation circuit consists of five separation stages, as follows: rougher (Ro), rougher-scavenger (Ro-Sc), cleaner $1(\mathrm{Cl} 1)$, cleanerscavenger $(\mathrm{Cl}-\mathrm{Sc})$, and cleaner $2(\mathrm{Cl} 2)$. Please note that these designations do not follow the rigorous functional unit descriptions outlined above. Table 4-4 summarizes the unit recovery values, feed grade, and other input values as well as the overall circuit performance. In this case, circuit recovery was determined by simple mass balance, and circuit uncertainty parameters (i.e. variance) was determined by applying LPE to the circuit analytical solution derived from the matrix reduction algorithm (Noble and Luttrell 2014). A constant standard deviation of $10 \%$ of the mean value was applied to each input parameter. By using the analytical circuit solution to determine overall circuit recovery, concentrate grade $\left(G_{C}\right)$ is given by: 
Table 4-4: Separation performance in separation stages and the original copper circuit. Input parameters based on Sepulveda et al., 2014. Output values calculated using mass balance and LPE.

\begin{tabular}{|l|l|l|}
\hline Parameter & Copper & Gangue \\
\hline Input Parameters & & \\
\hline Recovery: Rougher, Ro (\%) & 73.51 & 3.53 \\
\hline Recovery: Rougher - scavenger, Ro - Sc (\%) & 16.72 & 1.12 \\
\hline Recovery: Cleaner - scavenger, Cl - Sc (\%) & 81.85 & 18.63 \\
\hline Recovery: Cleaner 1, Cl 1 (\%) & 76.85 & 31.69 \\
\hline Recovery: Cleaner 2, Cl 2 (\%) & 78.92 & 53.35 \\
\hline Feed Grade & 0.085 & -- \\
\hline & & \\
\hline Output Values & & \\
\hline Circuit Recovery (\%) & 76.58 & 0.90 \\
\hline Recovery Uncertainty (\% recovery points) & 6.52 & 0.15 \\
\hline Circuit Grade (\%) & 27.02 & -- \\
\hline Grade Uncertainty (\% grade points) & 4.20 & -- \\
\hline
\end{tabular}

$$
G_{C}=\frac{G_{F} \times R_{C}}{\left(G_{F} \times R_{C}\right)+\left(\left(100-G_{F}\right) \times R_{G}\right)}
$$

where $R_{C}$ is the circuit copper recovery, $R_{G}$ is the circuit gangue recovery, and $G_{F}$ is the circuit feed grade.

\subsubsection{Analysis of original circuit}

Comparison between uncertainty levels of various circuit performance indicators in Table 4-4 shows the comparatively low degree of overall copper recovery uncertainty (i.e. standard deviation, $8.51 \%$ of mean in this case) and high level of gangue recovery $(16.67 \%$ of mean) and concentrate grade uncertainty propagation (15.56\% of mean). To accurately identify influential parameters in the global circuit selectivity and uncertainty propagation, the LPE-LCA technique was utilized. 
Figure 4-6b shows the contribution indices (calculated from Eq. 18) of stage recoveries for overall copper and gangue recovery parameters. These data show that recovery in the rougher stage (Ro) is the most influential parameter with respect to overall copper recovery, while all other units contribute very little less than $1 \%$ to any observed variance. For example, a $10 \%$ increase in the value of copper recovery in the rougher results in $8.80 \%$ improvement of overall copper recovery. Alternatively, the similar level of copper recovery increase in $\mathrm{Cl} 2$ causes only $0.13 \%$ enhancement in circuit copper recovery rate. Likewise, a $50 \%$ decrease in the standard deviation of copper recovery in the rougher Ro results in a $48.55 \%$ reduction in overall circuit copper recovery uncertainty. Alternatively, a $50 \%$ decrease in the standard deviation of $\mathrm{Cl} 2$ copper recovery only produces a $0.0001 \%$ reduction for the overall circuit. Interestingly, while the copper recovery in $\mathrm{Cl} 1$ and $\mathrm{Cl} 2$ separation stages have relatively no impact on the overall copper recovery, these factors are two of the most significant parameters in determining the mean and standard deviation of circuit gangue recovery rate. It can be concluded that, in these separation stages, a decline in the recovery rate of both copper and gangue materials results in a dramatic reduction of circuit gangue recovery, but a negligible change in copper recovery and thus, a positive change in the circuit concentrate grade. Moreover, Figure 4-6b indicates that Ro-Sc and ClSc separation stages are insignificant in defining the mean and standard deviation of copper and gangue recovery rates when compared with the other three separation stages.

Additionally, given uncertain stage recovery and feed grade factors, LPE-LCA methodology was employed to identify the most influential parameters in estimating the concentrate grade mean and standard deviation (Figure 4-6c). The calculated contribution indices show the importance of gangue recovery in $\mathrm{Cl}$ 1, Ro, and $\mathrm{Cl} 2$ for determining the mean and standard deviation of the concentrate grade factor. These data also indicate that feed grade and copper recovery in Ro separation stage play a significant role in estimating circuit concentrate grade and its variation. Once again, it can be observed that Ro-Sc separation stage has no noteworthy influence on the selectivity and uncertainty propagation in the copper circuit. 


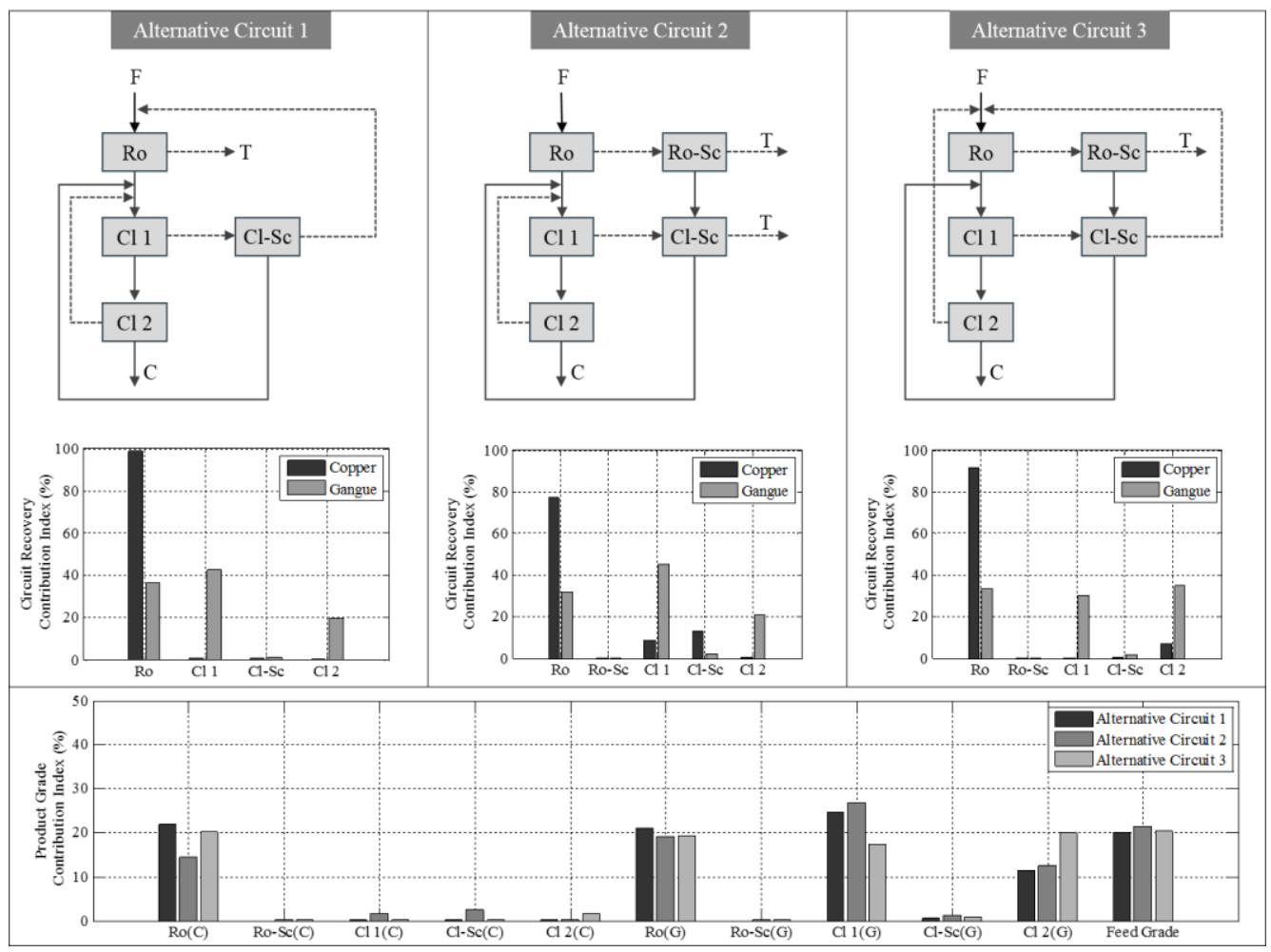

Figure 4-7: Alternative copper circuit configurations along with separation unit contribution indices for copper and gangue recovery factors and concentrate grade parameter.

\subsubsection{Analysis of alternative circuits}

To validate the rules and observations described in prior sections of this chapter, three alternative copper processing circuit designs were examined using similar assumptions as those used in the base circuit case. Like the base case, unit recovery values were used to determine the circuit recovery via mass balance, and LPE was used to determine the circuit uncertainty. The cumulative results from this alternative circuit analysis are shown in Table 4-5 and specific details are discussed in greater detail below. Each circuit was first screened using the rules derived above and the circuits were then assessed using the GSA methodology. 
Table 4-5: Separation performance of copper alternative circuits.

\begin{tabular}{|c|c|c|c|c|}
\hline Circuit designs & Base Circuit & $\begin{array}{l}\text { Alternative } \\
\text { Circuit } 1\end{array}$ & $\begin{array}{l}\text { Alternative } \\
\text { Circuit } 2\end{array}$ & $\begin{array}{l}\text { Alternative } \\
\text { Circuit } 3\end{array}$ \\
\hline Modification & --- & $\begin{array}{l}\text { Removed scavenger } \\
\text { functional unit (Unit } \\
\text { Ro-SC); replaced a } \\
\text { Scavenger-cleaner } \\
\text { functional unit (Cl- } \\
\text { Sc) with a scavenger } \\
\text { functional unit. }\end{array}$ & $\begin{array}{l}\text { Replaced a } \\
\text { rougher- } \\
\text { scavenger (unit: } \\
\text { Ro) with rougher } \\
\text { functional unit }\end{array}$ & $\begin{array}{l}\text { Replaced a cleaner- } \\
\text { scavenger (unit } \mathrm{Cl} \\
\text { 1) with cleaner } \\
\text { functional unit }\end{array}$ \\
\hline $\begin{array}{l}\text { Anticipated } \\
\text { Change based } \\
\text { on Section } 4.4 .1 \\
\text { Rules }\end{array}$ & --- & $\begin{array}{l}\text { Increase of copper } \\
\text { recovery uncertainty; } \\
\text { minor decrease of } \\
\text { gangue recovery } \\
\text { uncertainty }\end{array}$ & $\begin{array}{l}\text { Increase of } \\
\text { copper recovery } \\
\text { uncertainty }\end{array}$ & $\begin{array}{l}\text { Increase of copper } \\
\text { recovery uncertainty }\end{array}$ \\
\hline $\begin{array}{l}\text { Copper } \\
\text { recovery }(\%)\end{array}$ & 76.58 & 72.19 & 72.14 & 72.35 \\
\hline $\begin{array}{l}\text { Uncertainty - } \\
\text { copper rec. }(\%)\end{array}$ & 6.52 & 7.62 & 6.75 & 7.52 \\
\hline $\begin{array}{l}\text { Gangue } \\
\text { recovery }(\%)\end{array}$ & 0.90 & 0.85 & 0.87 & 0.75 \\
\hline $\begin{array}{l}\text { Uncertainty - } \\
\text { gangue rec. }(\%)\end{array}$ & 0.15 & 0.14 & 0.15 & 0.13 \\
\hline $\begin{array}{l}\text { Product grade } \\
(\%)\end{array}$ & 27.02 & 27.16 & 26.59 & 29.6 \\
\hline $\begin{array}{l}\text { Uncertainty - } \\
\text { Product grade } \\
(\%)\end{array}$ & 4.20 & 4.44 & 4.22 & 4.62 \\
\hline
\end{tabular}

Given the minor influence of the Ro-Sc unit that was observed in the prior section, alternative circuit 1 (Figure 4-7) was selected to evaluate the case where that unit is removed completely. As illustrated in Table 4-5, elimination of Ro-Sc separation stage results in an overall recovery reduction of $4.39 \%$ points and slight concentrate grade improvement of $0.14 \%$. With respect to recovery uncertainty/variability, the rules in in section 4.4 .1 provide two conflicting outcomes. The loss of a strict scavenging unit resembles the inverse of the change described in the first row of Table 4-2. This row shows that adding scavenging units tends to decrease the uncertainty in the recovery of ore material, while increasing the uncertainty of the recovery of gangue material. Since this modification is inverse change (i.e. removing rather than adding a scavenging unit), the outcome should also be reversed (i.e. ore recovery uncertainty is increased, while gangue recovery uncertainty is decreased). 
However, a second, more subtle change in this circuit is that the removal of the Ro-Sc unit also causes the $\mathrm{Cl}-\mathrm{Sc}$ unit to be converted from a cleaner-scavenger functional unit to a strict scavenger functional unit. This change is mirrored by the C6-C7 circuit comparison where a scavenger functional unit is modified to a scavenger-cleaner unit. Taking the inverse of the data in Table 4-3 suggests that current scavenger-cleaner to scavenger modification will produce a slight increase to the uncertainty in gangue particles and no change to the uncertainty in ore particles.

Thus, if these two outcomes are additive, the total result of the two simultaneous changes will be that the uncertainty in the recovery ore particles will increase substantially, while the uncertainty in the recovery of gangue particles will either reduce slightly (based on the overwhelming influence of the scavenger removal change relative to the smaller change due to cleaner-scavenger modification) or stay approximately equal to that of the base circuit. Overall, this expectation was validated, as the simulation showed that copper recovery uncertainty increased from $6.52 \%$ to $7.62 \%$ points, while gangue recovery only changed a negligible amount $(0.01 \%$ points). Finally, the GSA analysis shows that the contribution indices of the separation stages in alternative circuit 1 indicate that elimination of Ro-Sc stage does not reflect considerable changes in the behavior of various separation stages and their impact on the overall circuit performance selectivity and uncertainty.

Alternative circuit 2 shows another modification where the Cl-Sc tailings streams is directed to final tailings, rather than to the rougher feed as is the case in the original circuit. This slight modification effectively changes the rougher-scavenger function unit (base case) to a single function rougher unit (Alternative circuit 2). In terms of raw performance, this loss of scavenging capacity is expected to reduce recovery, and the simulation results confirm this expectation (i.e. copper recovery was reduced from $76.58 \%$ to $72.14 \%$, while grade was also reduced from $27.02 \%$ to $26.59 \%$ ). In terms of recovery uncertainty, the first row of Table 4-3 suggests that the modifying a rougher unit to a rougher-scavenger unit is expected to increase uncertainty of high recovery particles, while having a minimal influence on gangue recovery uncertainty. The current modification represents the inverse of this rule (i.e. replacing a rougher scavenger unit with a sole rougher unit), and the corresponding data validates the expectation. As shown in Table 4-5, the modification from the base circuit to Alternative Circuit 2 increased the copper recovery uncertainty from $6.52 \%$ to $6.75 \%$, while 
the gangue recovery uncertainty remained unchanged at $0.15 \%$. According to GSA analysis shown in Figure 4-7 and the data in Table 4-5, this modification considerably reduces the importance of Ro stage in estimating the circuit performance selectivity and uncertainty.

Finally, alternative circuit 3 is introduced, as a similar circuit to the original circuit configuration with only one difference in unit interconnections. In this alternative circuit, the cleaner-scavenger functional unit is replaced by the cleaner functional unit, and the rougher-scavenger functional unit is given an additional feed stream. Although this simple modification results in $4.23 \%$ copper recovery rate reduction, it considerably improves concentrate grade by $2.60 \%$. Moreover, as suggested by the rule in section 4.4 .1 , the level of circuit uncertainty propagation for the copper recovery is increased due to elimination of scavenging functionality from the cleaner-scavenger functional unit. Sensitivity analysis of this circuit using LPE-LCA approach indicates that the degree of influence that $\mathrm{Cl} 1$ separation stages have on the final selectivity and uncertainty propagation varied. $\mathrm{Cl} 1$, as a cleaner-scavenger functional unit in the original circuit configuration, projects a lower level of impact on the circuit selectivity and uncertainty in the modified circuit configuration. These results, all together, verifies the importance of functional unit utilization on the circuit performance indicators.

\subsection{Summary and conclusions}

Evaluation of the mineral processing circuit performance under uncertainty is an essential part of an effective circuit design methodology. Due to the distinct characteristics of separation circuits, including rigidity and difficulties associated with plant modifications, a practical circuit design and evaluation technique must be capable of explaining fundamental trends and expectations, so that these rules can be implemented during the early design stages. In the current study, the LPE-LCA methodology was combined with the fundamentals of process functional units to investigate the impact of separation unit operation and recirculating loads on the separation circuit uncertainty propagation. Subsequently, to more effectively evaluate the role of stage recovery and feed grade factors on the circuit selectivity and uncertainty propagation, LPE-LCA was employed as a GSA method. From that analysis, the contribution index was defined to show which units most influence the overall variance in circuit performance metrics. 
The following conclusions are drawn from this chapter:

- The direction and degree of circuit uncertainty propagation can be readily predicted using the functional unit concept.

- The cleaner functional unit intensifies the level of recovery uncertainty for particle classes of higher recovery values while suppressing the uncertainty propagation for the lower recovery particle classes. On the other hand, the scavenger unit operation shows opposite behaviors under uncertain conditions.

- Addition of cleaning function to units that already perform rougher or scavenging functions tends to decrease the recovery uncertainty of gangue particles (i.e. particles with low recovery values), while producing an insignificant change to the uncertainty of valuable particles. The inverse trend is seen for the scavenging function.

- Based on the prior two conclusions: dual and triple functional units tend to reduce overall circuit uncertainty; however, that reduction may be more strongly emphasized for certain particle classes.

- Furthermore, while the multi-function units reduce overall uncertainty, these units tend to be the most influential in determining circuit performance. Often this influence is related to the number of functions the unit is performing.

Lastly, these conclusions are based on a limited number of observations presented herein. The concept of functional unit evaluation as a metric to assess circuit performance and uncertainty propagation merits further consideration and validation in future studies.

\subsection{Bibliography}

Amini SH, Noble A. Application of linear circuit analysis in evaluation of mineral processing circuit design under uncertainty. Minerals Engineering. Elsevier; 
2017;102:18-29.

Ghaffari A, Hayati M, Shekholeslami A. Probability and Sensitivity Analysis in Flotation Circuit of Bama Lead and Zinc Processing Plant Using Monte Carlo Simulation Method. Mineral Processing and Extractive Metallurgy Review. Taylor \& Francis; 2012;33(6):416-26.

Hay M, Martin C. SUPASIM: A methodology to predict plant performance from laboratory data. Proceedings 36th Annual Meeting of the Canadian Mineral Processors. 2004. p. 281-99.

Jamett N, Cisternas LA, Vielma JP. Solution strategies to the stochastic design of mineral flotation plants. Chemical Engineering Science. Elsevier; 2015;134:850-60.

Karamoozian M, Shafaei S, Kakaie R, Noaparast M, Zeidabadi S, Ghasemi S. SAG mill power draw simulation using variance propagation approach. Iranian Journal of Science and Technology, Transaction B, Engineering. 2008;32(B6):619-29.

Meloy T. Analysis and optimization of mineral processing and coal-cleaning circuits-circuit analysis. International Journal of Mineral Processing. Elsevier; 1983 a;10(1):61-80.

Meloy T. Optimizing for grade or profit in mineral processing circuits-Circuit analysis. International journal of mineral processing. Elsevier; 1983 b;11(2):89-99.

Meloy TP, Clark N, Glista JJ. Effect of density variations in heavy-media rougher-cleanerscavenger cells-circuit analysis. International journal of mineral processing. Elsevier; 1986;16(3):169-78.

Mendez DA, Gálvez ED, Cisternas LA. State of the art in the conceptual design of flotation circuits. International Journal of Mineral Processing. Elsevier; 2009;90(1):1-15.

Montenegro MR, Sepúlveda FD, Gálvez ED, Cisternas LA. Methodology for process analysis and design with multiple objectives under uncertainty: Application to flotation circuits. International Journal of Mineral Processing. Elsevier; 2013;118:15-27.

Napier-Munn T. Statistical methods for mineral engineers-How to design experiments and analyse data. Julius Kruttschnitt Mineral Research Centre; 2014. 
Noble A, Luttrell G. Value-based objective functions applied to circuit analysis. Minerals \& Metallurgical Processing. 2015;32(1).

Noble A, Luttrell GH. The matrix reduction algorithm for solving separation circuits. Minerals Engineering. Elsevier; 2014;64:97-108.

Noble CA. Analytical and Numerical Techniques for the Optimal Design of Mineral Separation Circuits. Virginia Tech; 2013;

Sepúlveda FD, Cisternas LA, Gálvez ED. Global sensitivity analysis of a mineral processing flowsheet. In: Andrzej Kraslawski and Ilkka Turunen, editor. Proceedings of the 23rd European Symposium on Computer Aided Process Engineering-ESCAPE 23, Lappeenranta, Finland: Elsevier; 2013. p. 913-8.

Sepúlveda FD, Cisternas LA, Gálvez ED. The use of global sensitivity analysis for improving processes: Applications to mineral processing. Computers \& Chemical Engineering. Elsevier; 2014;66:221-32.

Williams M, Fuerstenau D, Meloy T. Circuit analysis-general product equations for multifeed, multistage circuits containing variable selectivity functions. International Journal of Mineral Processing. Elsevier; 1986;17(1):99-111.

Williams M, Meloy T. Dynamic model of flotation cell banks-circuit analysis. International Journal of Mineral Processing. Elsevier; 1983;10(2):141-60.

Williams M, Meloy T. On the definition and separation of fundamental process functions. International Journal of Mineral Processing. Elsevier; 1989;26(1):65-72.

Xiao Z, Vien A. Limitations of variance analysis using propagation of variance. Minerals engineering. Elsevier; 2003;16(5):455-62. 


\title{
Chapter 5
}

\section{Experimental Validation of Circuit Uncertainty Quantification Methodology}

\begin{abstract}
(ABSTRACT)
A systematic experimental study was performed to validate the analytical methods and conclusions proposed in prior chapters. For these experiments, a roll-type corona electrostatic separator was used to separate $\mathrm{Ti}$ and $\mathrm{Si}$ in a heavy mineral sand consisting of illmenite, zircon, rutile, and silica. Several multi-unit circuit configurations were evaluated by operating the laboratory unit in a locked-cycle fashion. The separation tests were carried out in triplicate and then the selectivity (i.e. mean) and uncertainty (i.e. standard deviation) measures were determined for several separation performance indicators. The comparison between the level of uncertainty propagation for distinct separation circuits indicated that the novel circuit uncertainty quantification technique provides exceptional capacity to evaluate separation circuit designs under uncertainty.
\end{abstract}

Keywords: electrostatic separator; heavy mineral sand; linear circuit analysis; law of propagation of error, functional unit 


\subsection{Introduction}

\subsubsection{Background}

As extensively reviewed in Chapter 2, over the last decade, several studies have addressed the topic of uncertainty in the mineral processing operation and separation circuit design procedure. Some of the prior studies, including Xiao and Vien (2003); Karamoozian et al. (2008); and Ghaffari et al. (2012), have emphasized on the significance of parameters' uncertainty and have assessed the influence of uncertainty propagation in flotation and comminution circuits utilizing Monte Carlo simulation. More recently, several studies have investigated the impact of stochastic input data on the separation performance for various circuit configurations (Lucay et al. 2012; Sepúlveda et al. 2014; Montenegro et al. 2015). Additionally, Montenegro et al. (2013) and Jamett et al. (2015) have demonstrated novel methods of integrating uncertain parameters in a more comprehensive and rigorous circuit optimization protocol. Despite these scientific developments, none of the studies include a comprehensive empirical validation of techniques for determination of the circuit uncertainty propagation.

\subsubsection{Analytical circuit quantification techniques}

Given the principles of linear circuit analysis approach (LCA) and law of propagation of error (LPE), this author has developed a novel approach to be used for the circuit uncertainty quantification (Amini and Noble 2017). Results of the proposed tool indicated that separation circuit configurations can intensify or suppress the level of uncertainty for various separation performance indicators. The LCA-LPE method only requires the stream connection diagram and estimates of the mean and uncertainty of input parameters. The accuracy of this analytical method was then investigated through the systematic comparisons against Monte Carlo simulation results.

Following the results of the initial analytical study, the LCA-LPE method was combined with the fundamentals of functional units to effectively evaluated separation circuit designs under uncertainty during the early design stages (Chapter 4). This innovative approach was employed to derive several generic design rules for predicting circuit uncertainty propagation 
outcomes. According to these rules, while the addition of scavenging capacity to the original circuit reduces the uncertainty of high recovery particles and intensifies the recovery uncertainty for low recovery particles, adding the cleaning capacity produce an identical, albeit reversed, trend. Finally, the applicability of this methodology was demonstrated through numerous generic circuit design examples and several industrial circuit configurations.

The LCA methodology is fundamentally restricted by the assumption of process linearity specifying that a unit's separation performance is not affected by particle-particle interactions. Additionally, the LPE mathematic formulation is determined according to Taylor series while neglecting higher order terms. The formulation is constructed based on the basic assumption underpinning the independency of the input parameters. As a result, despite the theoretical effectiveness and accuracy of the proposed analytical methodologies, there is an essential need for the verification of these novel analytical techniques via comprehensive empirical separation experiments.

\subsubsection{Experimental validation program}

To validate the capability of the proposed analytical circuit uncertainty determination tool, a systematic separation tests was carried out using a roll-type corona electrostatic separator. Several separation circuit configurations were simulated employing locked cycle experiments. Each run was repeated several times to assess circuit variability/uncertainty by determining both the mean and variance of several performance indicators. Finally, the data comparisons between the separation performance of representative circuit configurations were utilized to verify the established design rules derived from the LCA-LPE tool for cases of uncertainty. 
Table 5-1: Assay analysis and electrostatic characteristics of heavy mineral sand sample.

\begin{tabular}{|c|c|c|c|}
\hline Element & Grade (\%) & Conductive & Non-conductive \\
\hline $\mathrm{Ti}$ & $31.08 \pm 4.62$ & $\mathrm{x}$ & \\
\hline $\mathrm{Si}$ & $20.59 \pm 1.59$ & & $\mathrm{x}$ \\
\hline $\mathrm{Zr}$ & $9.92 \pm 4.84$ & & $\mathrm{x}$ \\
\hline $\mathrm{Fe}$ & $10.57 \pm 0.57$ & $\mathrm{x}$ & \\
\hline $\mathrm{Al}$ & $8.37 \pm 1.39$ & & $\mathrm{x}$ \\
\hline Total & 100.00 & & \\
\hline
\end{tabular}

\subsection{Experimental materials and methods}

\subsubsection{Materials}

\section{Heavy mineral sample characteristics}

The heavy mineral sand sample used throughout the experimental testing was obtained from a standard dealer. Table 5-1 shows the feed assay for the heavy mineral sand sample. These data indicate that the $\mathrm{Ti}$ and $\mathrm{Si}$ concentrations in the heavy mineral sample are $31.58 \%$ and $20.84 \%$, respectively. Additionally, the electrostatic properties of these illustrated elements indicate that the electrostatic separator should be capable of concentrating $\mathrm{Ti}$ and $\mathrm{Fe}$ in the conductive product hopper while reporting $\mathrm{Si}, \mathrm{Al}$, and $\mathrm{Zr}$ to the Tailings (nonconductive) stream. Furthermore, the size analysis of the feed sample (Figure 5-1) reveals that all particles were in the $50 \times 600$ microns size fraction.

\section{Electrostatic separator}

For the purpose of this study, a laboratory-scale roll-type corona electrostatic separator (Figure 5-2) was used as an effective technology for separating conductive and nonconductive materials. Electrostatic separators have been widely used in mineral processing operations (Fuerstenau and Han 2003; Wills 2011), coal cleaning applications (Bada et al. 2010), and recycling of metals from plastics in industrial wastes (Li et al. 2007; Tilmatine et al. 2009). These dry-based concentrators segregate materials based on their differential electrical conductivity by the means of a high voltage electric field. 


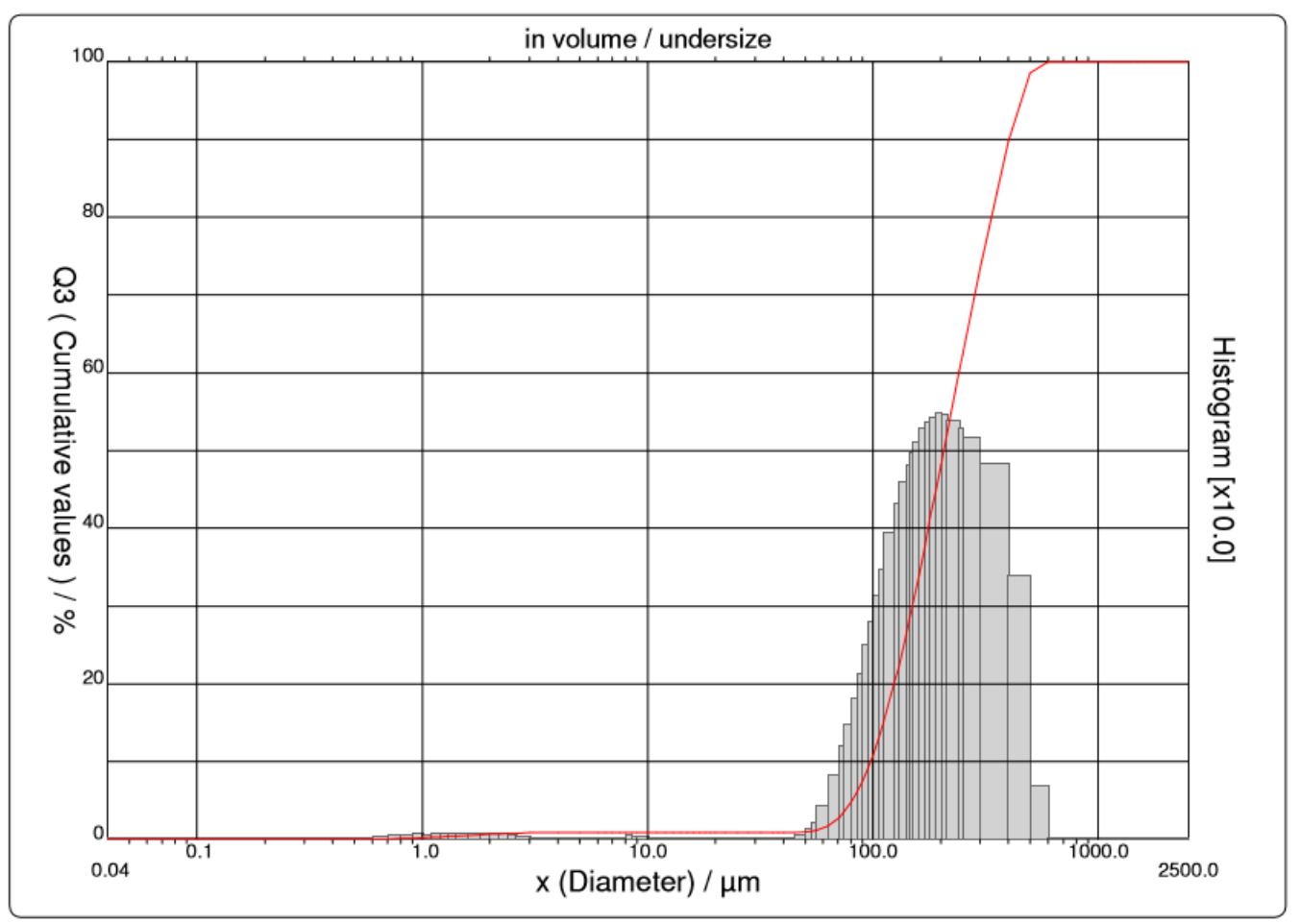

Figure 5-1: Size-by-size analysis of the heavy mineral sand sample.

Figure 5-3 and 5-4 shows the laboratory-scale and schematic representation of an electrostatic separator and demonstrate various apparatus parts. The corona electrostatic separator employs the mechanism of ion bombardment (corona charging) to charge minerals and then separate particles on the basis of electrical properties. Charging via ion bombardment occurs as a high voltage is applied between two electrodes, thereby producing a continuous flow of gaseous ions, a corona discharge, near the electrodes (Wills and Finch 2015). During normal operations, the particulate feed mixture is fed onto the surface of the rolling drum through the material feeder with adjustable flow rate option. Due to the detrimental impact of particles' humidity on the separation efficiency, the material feeder is provided with the elevated-temperature heater. The perfectly dried mineral particles passing through the corona are bombarded with the flow of ions and develop a charge. Particles with different electrical conductivity maintain distinct levels of surface charge. As a result, while non-conductive particles with a high degree of surface charge are attracted and pinned to the rotor surface, conductive particles are thrown from the drum due to the centrifugal force of 


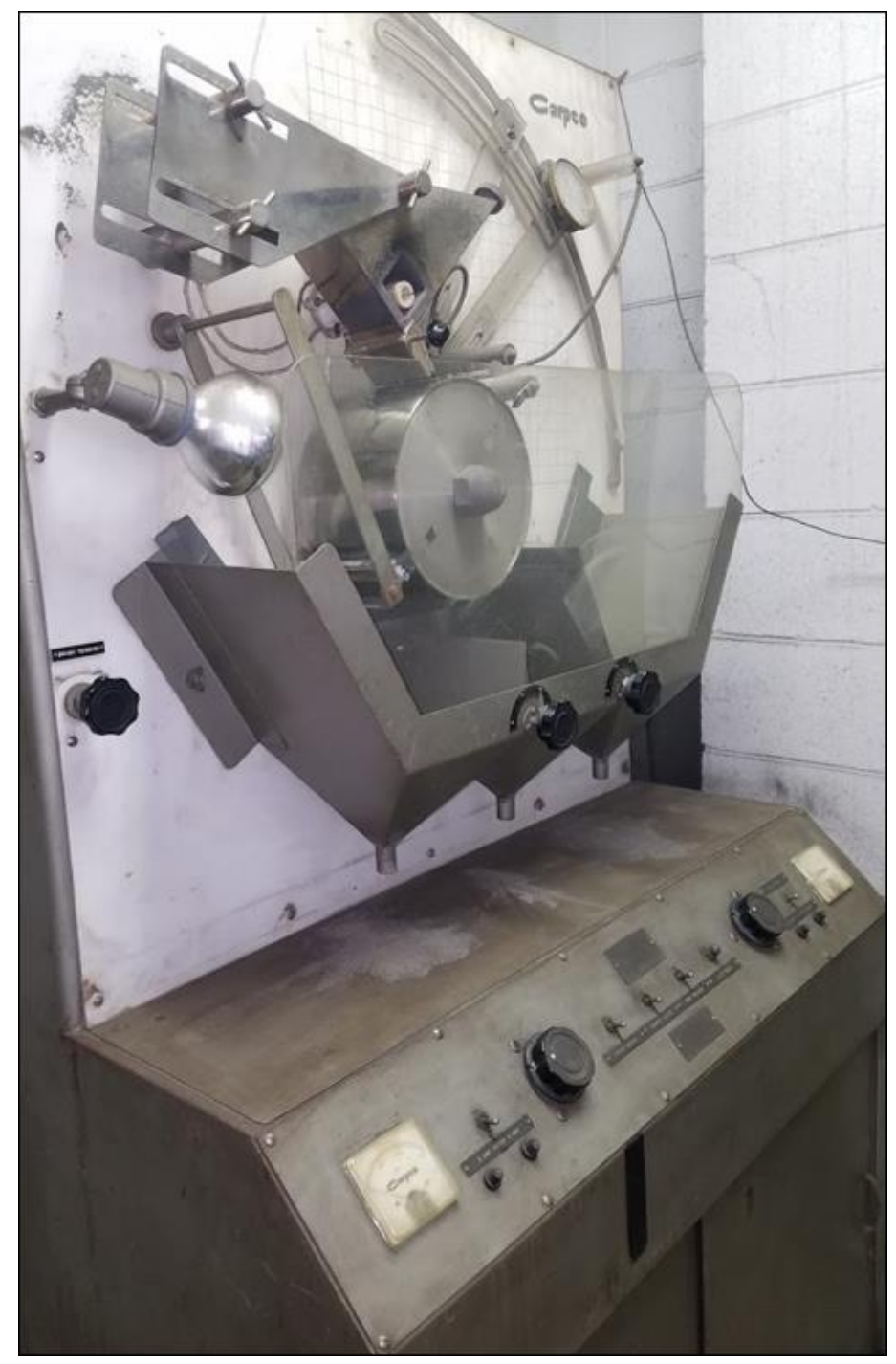

Figure 5-2: Laboratory-scale roll-type corona electrostatic separator.

the drum and gravitational as well as frictional forces. Therefore, two distinct streams of particles are produced which then may be collected separately by the means of splitters. In the current study, an electrostatic separator is employed to demonstrate the performance of various open and recycled circuit configurations in separating $\mathrm{Ti}$, as the conductive particle, from $\mathrm{Si}$, as the non-conductive particle, in a mixture of heavy mineral sand. 


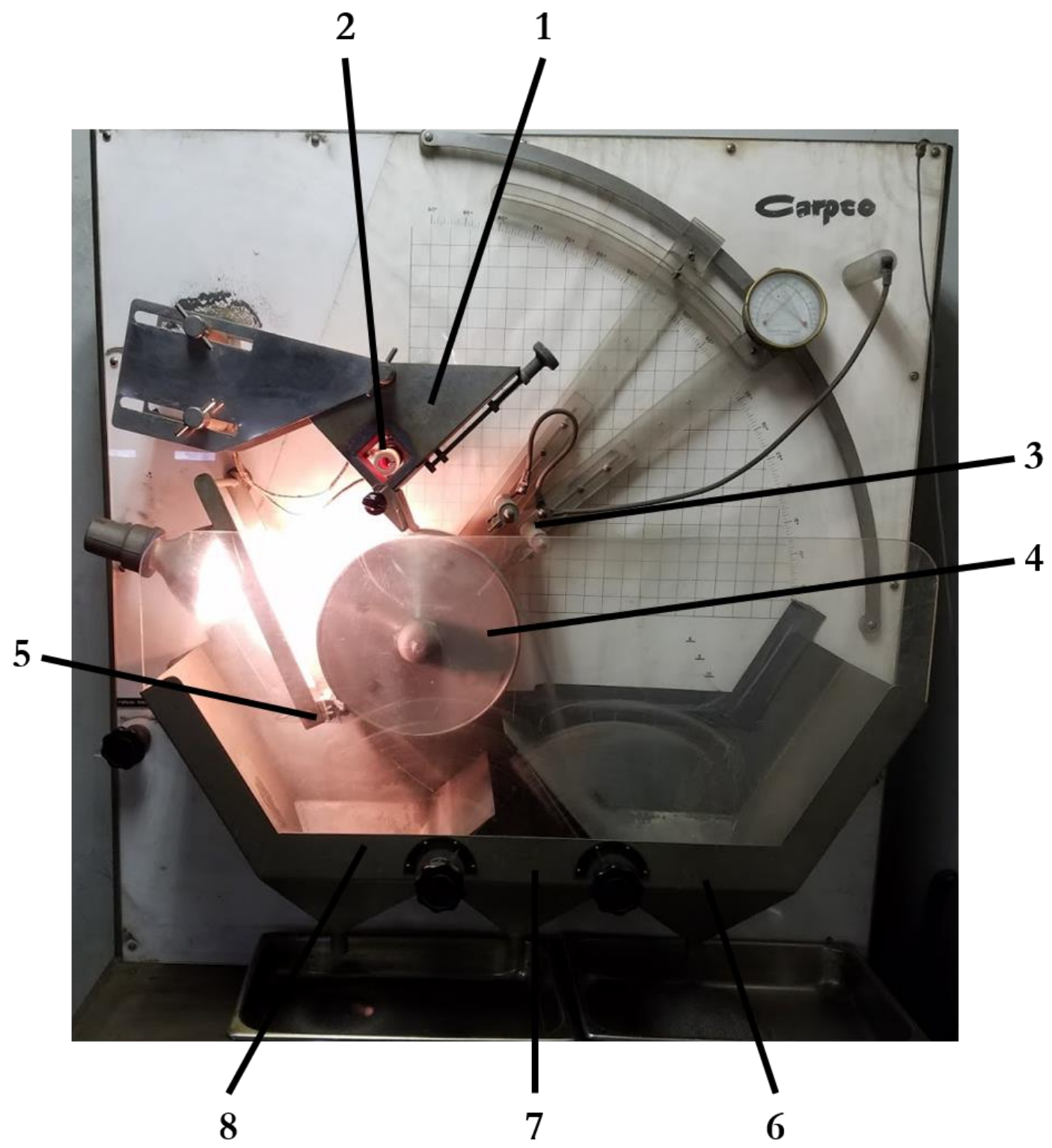

Figure 5-3: Laboratory-scale roll-type corona electrostatic separator: (1) material feeder; (2) feed heater; (3) electrodes; (4) rolling drum; (5) brush; (6) conductive material hopper; (7) middling material hopper; (8) non-conductive material hopper. 


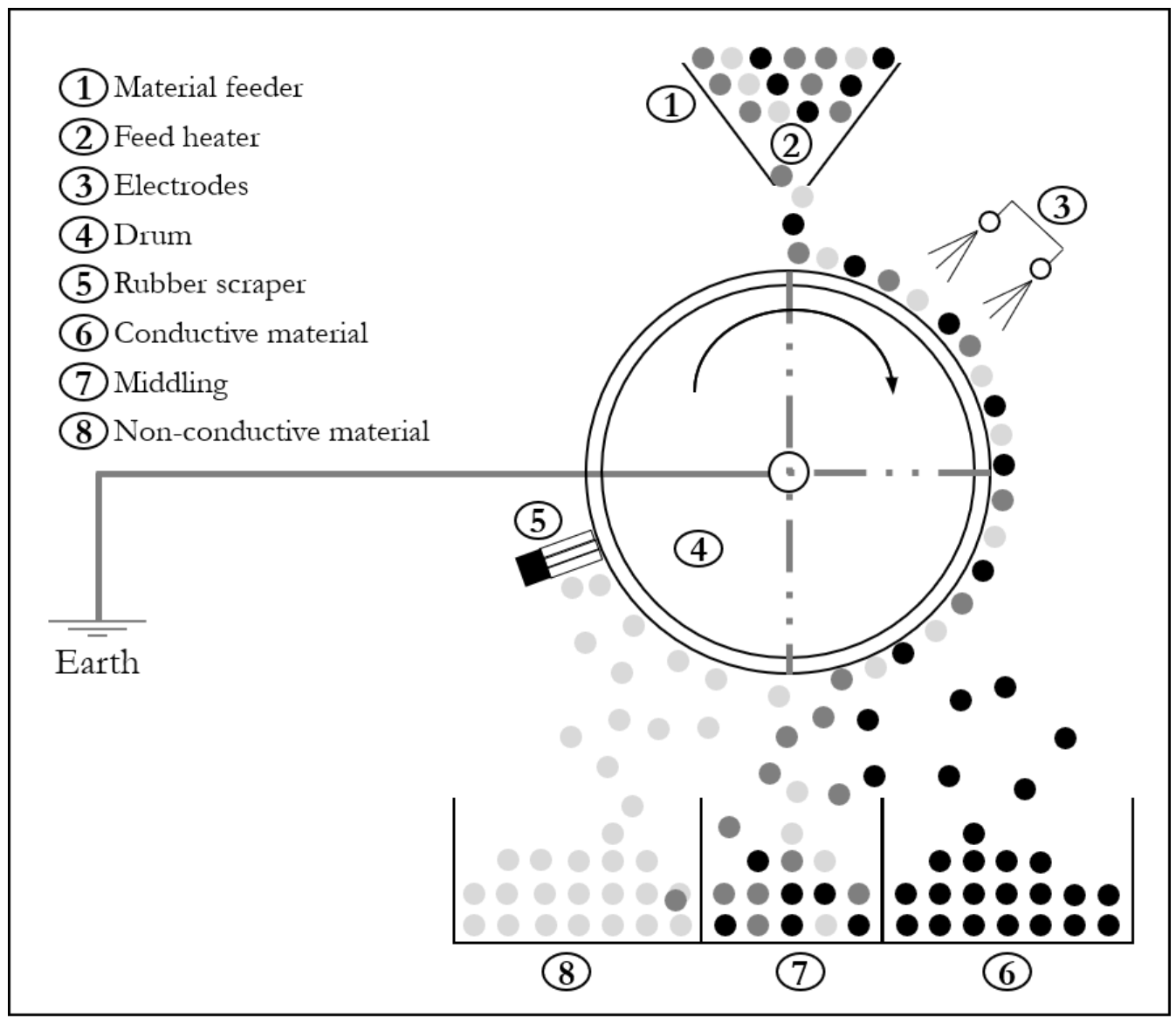

Figure 5-4: Descriptive schematic of the role-type corona electrostatic separator.

\subsubsection{Methods}

\section{Laboratory procedure for separation tests}

As extensively described in the prior section, separation performance tests were conducted using a laboratory-scale electrostatic separator. To prepare for each test, a sufficient quantity of feed sample (250 to 350-grams) was representatively taken from the original heavy mineral sand sample source. Afterwards, a small (approximately 5-grams) subsampled was recovered from this feed and then analyzed to determine the feed assay. After the feed sample preparation, the position of product hopper outlets (splitters) in the electrostatic separator were adjusted to distinguish between the separation performance of 


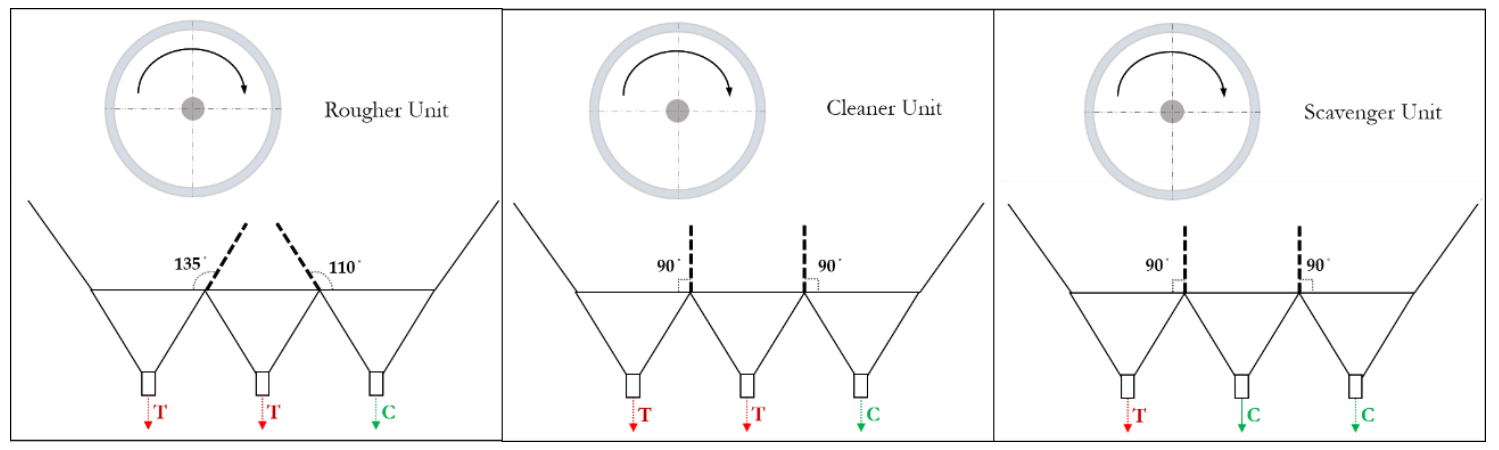

Figure 5-5: The location of product hopper outlets for rougher, scavenger, and cleaner units.

the rougher, scavenger, and cleaner units. Figure 5-5 depicts the product and tailing splitter angles for each distinct separation stage. The electrostatic separator was then allowed to run at the high voltage level of $40 \mathrm{~kW}$ and the rotating drum speed of 70-rpm. The heavy mineral sand sample was then added to the material feeder and the perfectly dried particles are fed to the rotating drum at the flow rate of approximately $100 \mathrm{gr} / \mathrm{min}$. After completion of each separation experiment, the conductive materials, including $\mathrm{Ti}$, and non-conductive particles, including $\mathrm{Si}$, were collected from the concentrate and tailings containers. Similar to the feed sample, the recovered concentrate and tailings materials were subjected to the weight measurement and chemical analysis. It is worth to note that throughout this study, the XRF spectrometer was used for the elemental quantification of feed, concentrate, and tailings materials. The XRF spectrometer is a non-destructive elemental analysis instrument that can be used for the fast $(30-60 \mathrm{sec})$ and accurate assay measurements. To confirm the accuracy of measurements, the assay analysis was repeated 10 times, the outlier values were eliminated, the statistical mean values were determined and then used for the further mass balance and data reconciliation.

To properly investigate the capability of LCA-LPE approach in predicting the circuit uncertainty quantification, several one-, two-, and three-unit circuit configurations were carefully selected (Figure 5-6). The separation performance comparisons among these circuit designs can be used to investigate the impact of scavenging and cleaning functionality on the degree and direction of circuit uncertainty propagation. A repetitive batch (locked-cycle) tests were carried out to simulate continuous circuits with either concentrate or tailings recycles. 


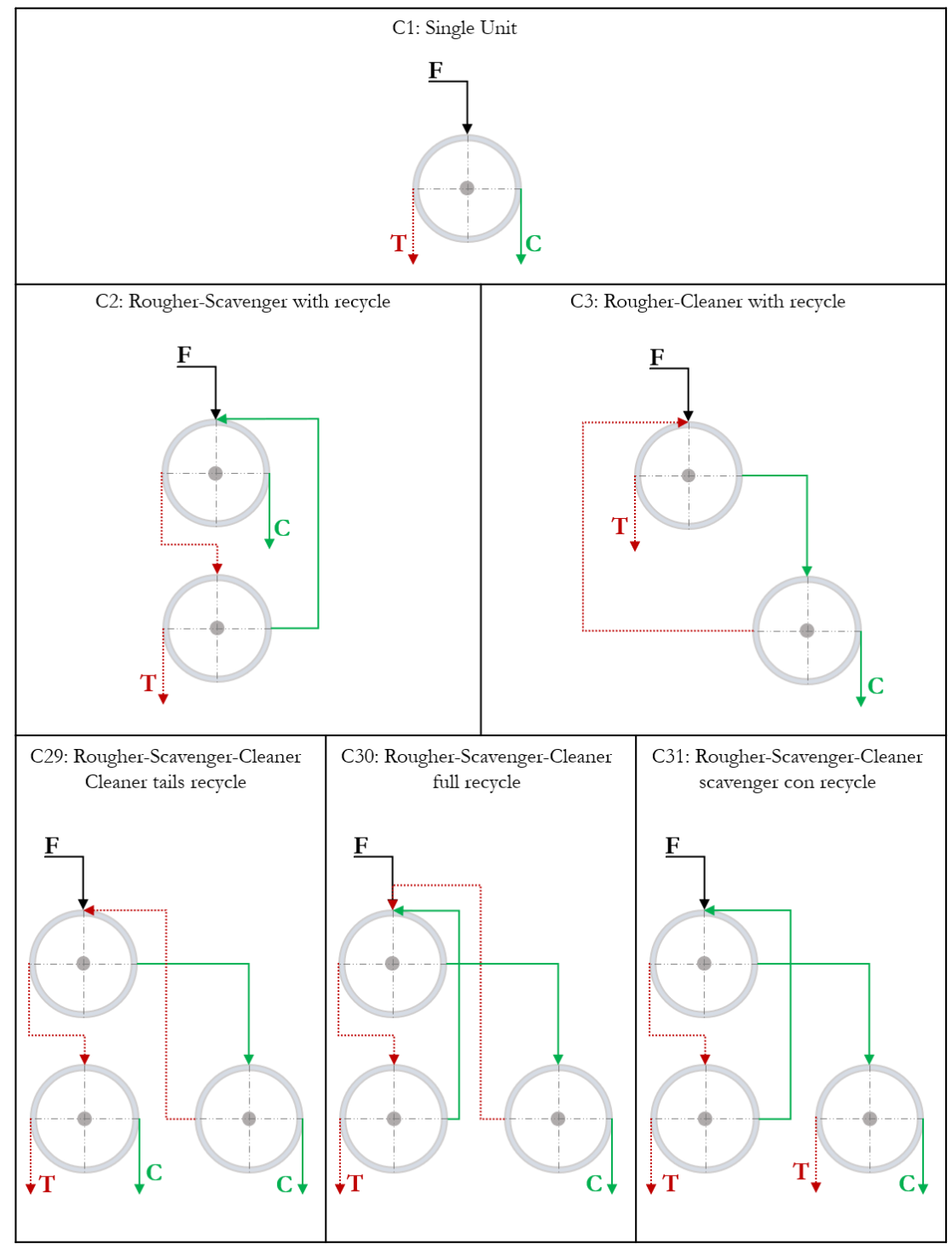

Figure 5-6: Selected circuit configurations for the separation experimental program.

The basic procedure had a complete batch test performed in the first cycle which was then followed by similar batch tests which had "intermediate" material from the previous cycle added to the appropriate location in the current cycle. These batch tests, or cycles, were continued in this iterative manner for an arbitrary number of cycles. To assess the circuit variability/uncertainty, the separation tests were repeated three times for each distinct circuit design. 


\section{Data analysis}

According to the resultant mass and assay measurements on the feed, concentrate, and tailings materials, the detailed mass balance calculation and data reconciliation were carried out to accurately determine the separation performance of individual circuit configurations. Initially, the mass yield parameter (i.e. mass recovery to the concentrate stream) was calculated based on the weight-based measurements of distinct streams. Given the accuracy of this estimation, weight-based estimation of yield parameter was considered as the reference measurement. Afterwards, the $\mathrm{Ti}$ and $\mathrm{Si}$ assay measurements were minimally modified in a way to satisfy the following equation:

$$
Y(\%)=\frac{C}{F} \times 100=\frac{(f-t)}{(c-t)} \times 100
$$

where $C$ and $F$ are the total weights of the concentrate and feed, respectively. In addition, $f, t$, and $c$ are the concentrations of elements under study (i.e. Ti or $\mathrm{Si}$ ). The data reconciliation procedure results in an accurate estimation of elemental assays in the feed, concentrate, and tailings streams.

The global circuit recovery of Ti and Si elements were estimated for all circuits. Here, recovery parameter $(R)$ is defined as:

$$
R(\%)=\frac{c \times(f-t)}{f \times(c-t)} \times 100
$$

Since all tests were performed in triplicate, the mean and standard deviation for the $\mathrm{Ti}$ and $\mathrm{Si}$ recovery indicators were calculated. Finally, meaningful comparisons were made between the recovery uncertainty of various circuits to investigate the validity of LCA-LPE findings. 
Table 5-2: Separation performance measures for experimental circuit configurations.

\begin{tabular}{|lcrr|ll|}
\hline Circuit & Run 1 & Run 2 & Run 3 & Mean & St. Dev. \\
\hline Ti Recovery (\%) \\
\hline C1 & 84.96 & 78.37 & 65.04 & 70.33 & 9.41 \\
C2 & 98.37 & 98.11 & 97.65 & 98.04 & 0.36 \\
C3 & 80.03 & 89.71 & 78.57 & 82.77 & 6.06 \\
C29 & 96.76 & 98.87 & 91.51 & 95.38 & 3.40 \\
C30 & 96.03 & 92.23 & 93.39 & 93.88 & 1.95 \\
C31 & 29.29 & 40.99 & 40.69 & 36.99 & 6.67 \\
\hline Si Recovery $\%)$ & \multicolumn{5}{|l}{} \\
\hline C1 & 28.70 & 25.90 & 16.13 & 20.68 & 6.60 \\
C2 & 50.97 & 34.69 & 40.47 & 42.04 & 8.25 \\
C3 & 9.17 & 6.94 & 7.54 & 7.88 & 1.16 \\
C29 & 28.09 & 20.52 & 10.26 & 19.62 & 8.94 \\
C30 & 11.44 & 12.70 & 7.34 & 10.49 & 2.80 \\
C31 & 5.23 & 4.22 & 3.68 & 4.37 & 0.79 \\
\hline Ti Grade (\%) & 42.93 & 43.07 & 38.83 & 41.61 & 2.41 \\
\hline C1 & 44.32 & 48.48 & 42.19 & 45.00 & 3.20 \\
C2 & 49.63 & 58.94 & 52.81 & 53.80 & 4.73 \\
C3 & 49.03 & 50.21 & 54.25 & 51.50 & 2.39 \\
C29 & 50.49 & \\
C30 & 55.42 & 51.85 & 56.48 & 54.58 & 2.43 \\
C31 & 47.36 & 48.33 & 48.11 & 47.93 & 0.51 \\
\hline
\end{tabular}

\subsection{Results and discussions}

Table 5-2 compiles the global recovery and concentrate grade data of three experimental runs (i.e. mean and standard deviation) for six individual circuit configurations. Overall, these data indicate that different circuit designs may display different behaviors, regarding the selectivity and uncertainty, treating the similar feed materials. Of all circuit performances, the superior separation performance of the rougher-scavenger-cleaner balanced circuit configuration (i.e. C30) is well-demonstrated. The level of uncertainty propagation in this circuit configuration is one of the lowest when compared with other five circuit designs. To 
Table 5-3: Circuit performance comparisons for evaluating the role of cleaning/scavenging capacity on the final circuit uncertainty propagation.

\begin{tabular}{|l|l|l|l|l|}
\hline $\begin{array}{l}\text { Type of Unit or } \\
\text { Functionality Addition }\end{array}$ & Base Circuit & Modified Circuit & $\begin{array}{l}\text { Impact on } \\
\text { Ti Rec. Uncertainty }\end{array}$ & $\begin{array}{l}\text { Impact on Si } \\
\text { Rec. Uncertainty }\end{array}$ \\
\hline $\begin{array}{l}\text { Scavenger } \\
\text { Functional Unit }\end{array}$ & C3 & C29 & -2.66 & +7.78 \\
\hline $\begin{array}{l}\text { Cleaner } \\
\text { Functional Unit }\end{array}$ & C2 & C31 & +6.31 & -7.46 \\
\hline $\begin{array}{l}\text { Scavenging } \\
\text { Functionality }\end{array}$ & C31 & C30 & -4.72 & 2.01 \\
\hline $\begin{array}{l}\text { Cleaning } \\
\text { Functionality }\end{array}$ & C29 & C30 & -1.45 & -6.14 \\
\hline $\begin{array}{l}\text { Scavenging \& Cleaning } \\
\text { Functionality }\end{array}$ & C1 & C31 & -7.46 & -3.8 \\
\hline
\end{tabular}

properly investigated the reliability of established LCA-LPE design rules and observations, the compounded level of recovery uncertainty for the representative circuit configurations were compared against one another. The cumulative results from this analysis are shown in Table 5-3 and specific details are discussed below.

\subsubsection{Scavenging capacity addition}

From the results of previous chapters (chapter 3 and 4), it concluded that the addition of scavenger functional unit results in intensifying the level of uncertainty for lower recovery particles while suppressing the recovery uncertainty for higher recovery particle classes. To verify this theoretical rule, the level of uncertainty for the $\mathrm{Ti}$ and $\mathrm{Si}$ recovery parameters in C3 and C29 circuit configurations were evaluated and compared. As shown in Table 5-3, adding scavenging capacity (scavenger functional unit) to the C3 leads in the dramatic rise in the level of uncertainty for the Si recovery while reduce the uncertainty of $\mathrm{Ti}$ recovery factor. Scavenging capacity may be added to the original circuit layout through the addition of tailings stream recirculation. As illustrated in Table 5-3, this modification causes a reduction in the value of uncertainty for Ti recovery in C30 when it compares with the performance of C31. These data confirm the theoretical observations previously described in Chapter 4 . 


\subsubsection{Cleaning capacity addition}

To further evaluate the derived rules by LCA-LPE methodology, the uncertainty associated with $\mathrm{Ti}$ and $\mathrm{Si}$ recovery factors were estimated and analyzed for C2, C29, and C31 circuit configurations. According to these comparisons, as proposed by the LCA-LPE methodology, when cleaner functional unit is added to the base circuit, the level of uncertainty for Si recovery intensely dropped. The comparison of compounded uncertainty for Ti recovery in C2 and C31 demonstrates the opposite trend. Similarly, recycling of the concentrate stream to the initial roughering stage in C29 (generating C30 circuit) results in a dramatic decrease in the uncertainty recovery of Si particles. Once again, the empirical separation experiments validate the established rules by the LCA- LPE approach.

As discussed in the previous chapter, the separation performance of $\mathrm{C} 1$ and $\mathrm{C} 30$ circuits can be compared to examine how the addition of both cleaning and scavenging capacities alter the level of circuit recovery uncertainty propagations. As indicated in Table 5-3, C31 circuit configuration produces lower level of uncertainty propagation for both $\mathrm{Ti}$ and $\mathrm{Si}$ recovery parameters when it compared with $\mathrm{C} 1$. Altogether, the fundamental circuit design rules for the cases of uncertainty, as previously proposed by the novel LCA-LPE technique, were confirmed through the experimental plan.

\subsection{Summary and conclusions}

This chapter has described an empirical separation experimental analysis that was employed to investigate the potential capability of the LCA-LPE approach in evaluating the circuit uncertainty quantification. As such, series of dry separation tests were conducted with an electrostatic separator treating heavy mineral feed sample. The uncertainty associated within the separation performance of six individual circuit configurations were determined. The data were used to validate the observations and established design rules derived from the LCA-LPE technique. As earlier suggested by the analytical circuit technique, following fundamental conclusions were drawn from this study. 
- Similar to the separation circuit selectivity, different circuit configurations may compound different levels of circuit uncertainty due to distinct unit interconnections and unit functionality.

- The LCA-LPE approach when combined with the basics of functional unit concept may be employed as a practical tool to determine and evaluate circuit uncertainty projection.

- The generic design rules on the addition of cleaning/scavenging capacity to the original circuit were shown to be reliable and can be employed in the preliminary stages of circuit design procedure.

\subsection{Bibliography}

Amini SH, Noble A. Application of linear circuit analysis in evaluation of mineral processing circuit design under uncertainty. Minerals Engineering. Elsevier; 2017;102:1829.

Bada S, Falcon R, Falcon L. The potential of electrostatic separation in the upgrading of South African fine coal prior to utilization-a review. Journal of the Southern African Institute of Mining and Metallurgy. The Southern African Institute of Mining and Metallurgy; 2010;110(11):691-702.

Fuerstenau MC, Han KN. Principles of mineral processing. SME; 2003.

Ghaffari A, Hayati M, Shekholeslami A. Probability and Sensitivity Analysis in Flotation Circuit of Bama Lead and Zinc Processing Plant Using Monte Carlo Simulation Method. Mineral Processing and Extractive Metallurgy Review. Taylor \& Francis; 2012;33(6):416-26.

Jamett N, Cisternas LA, Vielma JP. Solution strategies to the stochastic design of mineral flotation plants. Chemical Engineering Science. Elsevier; 2015;134:850-60.

Karamoozian M, Shafaei S, Kakaie R, Noaparast M, Zeidabadi S, Ghasemi S. SAG mill power draw simulation using variance propagation approach. Iranian Journal of Science and Technology, Transaction B, Engineering. 2008;32(B6):619-29.

$\mathrm{Li} \mathrm{J,} \mathrm{Xu} \mathrm{Z,} \mathrm{Zhou} \mathrm{Y.} \mathrm{Application} \mathrm{of} \mathrm{corona} \mathrm{discharge} \mathrm{and} \mathrm{electrostatic} \mathrm{force} \mathrm{to} \mathrm{separate}$ metals and nonmetals from crushed particles of waste printed circuit boards. Journal of 
Electrostatics. Elsevier; 2007;65(4):233-8.

Lucay F, Mellado ME, Cisternas LA, Gálvez ED. Sensitivity analysis of separation circuits. International Journal of Mineral Processing. Elsevier; 2012;110:30-45.

Montenegro MR, Gálvez ED, Cisternas LA. The effects of stage recovery uncertainty in the performance of concentration circuits. International Journal of Mineral Processing. Elsevier; 2015;143:12-7.

Montenegro MR, Sepúlveda FD, Gálvez ED, Cisternas LA. Methodology for process analysis and design with multiple objectives under uncertainty: Application to flotation circuits. International Journal of Mineral Processing. Elsevier; 2013;118:15-27.

Sepúlveda FD, Cisternas LA, Gálvez ED. The use of global sensitivity analysis for improving processes: Applications to mineral processing. Computers \& Chemical Engineering. Elsevier; 2014;66:221-32.

Tilmatine A, Medles K, Bendimerad S-E, Boukholda F, Dascalescu L. Electrostatic separators of particles: Application to plastic/metal, metal/metal and plastic/plastic mixtures. Waste management (New York, NY). Elsevier Ltd; 2009;29(1):228-32.

Wills BA. Wills' mineral processing technology: an introduction to the practical aspects of ore treatment and mineral recovery. Butterworth-Heinemann; 2011.

Wills BA, Finch J. Wills' mineral processing technology: an introduction to the practical aspects of ore treatment and mineral recovery. Butterworth-Heinemann; 2015.

Xiao Z, Vien A. Limitations of variance analysis using propagation of variance. Minerals engineering. Elsevier; 2003;16(5):455-62. 


\title{
Chapter 6
}

\section{Application of Taguchi's Method to Uncertainty Assessment for Mineral Processing Circuit Design}

\begin{abstract}
(ABSTRACT)
While many classic separation circuit design approaches rely on deterministic methods, numerous studies have shown that the uncertainty inherent to the various input parameters can significantly influence design decisions. In many processing applications, these individual uncertainties are propagated by the circuit design and reflected in the circuit performance indicators, such as product grade and global recovery. Unfortunately, few analytical tools provide information on uncertainty propagation, particularly those that rely solely on deterministic methods. However, one promising approach is through a novel implementation of Taguchi's method, in a fashion similar to statistical tolerancing of manufactured products. Taguchi's method shows promise in evaluating the level of compounded uncertainty by various circuit designs and requires significantly fewer function evaluations when compared to other stochastic methods, such as Monte Carlo simulation. This paper provides two examples of Taguchi's method as applied to mineral processing circuit design problems. The Taguchi's method results show excellent agreement with Monte Carlo simulation, but in both cases, the required number of function evaluations was reduced by several orders of magnitude.
\end{abstract}

Keywords: circuit design; uncertainty; Taguchi’s method; Monte Carlo simulation. 
(Note: This chapter was largely derived from the following publication and has been reformatted to departmental guidelines: Amini SH, Noble A. Application of Taguchi's method to uncertainty assessment and sensitivity analysis for mineral processing circuits; 2017 SME Annual Meeting \& Exhibit. Denver, CO. Preprint No 17-011.)

\subsection{Introduction and method description}

\subsubsection{Uncertainty in Mineral Processing Circuit Design}

Analysis and estimation of uncertainty propagation in mineral processing separation circuits is an essential and significant aspect of an optimal circuit design procedure. Most of the current circuit design methodologies have been constructed, implemented, and validated using a deterministic modeling approach. While this approach may be suitable for wellconstrained problems, numerous studies have shown that uncertainty induced by lack of knowledge (epistemic uncertainty) and stochastic variation (aleatory uncertainty) can both be significant factors in many mineral processing applications (Kraslawski 1989; Xiao and Vien 2003; Ghaffari et al. 2012). Thus, an effective circuit design methodology must be able to quantify and assess uncertainty in separation circuits. Most contemporary approaches rely on Monte Carlo simulation (MCS) to perform stochastic analysis, but this method can be limited due to long calculation times and cumbersome input requirements. This technical note describes an alternative approach to stochastic analysis using Taguchi's method. The calculation steps for the method are first described and two mineral processing design applications are presented. All results are last compared to similar data produced by MCS.

\subsubsection{Monte Carlo Simulation}

The term "Monte Carlo Simulation" has been used to refer to a variety of stochastic simulation methods spanning a broad array of business, technical, and scientific domains. Most generally, MCS refers to numerical analysis that is based on repeated (pseudo) random sampling from a domain of input parameters with a known statistical distribution. This methodology allows one to determine the distribution of expected results from a mathematical function (i.e. a deterministic process model) that is dependent on the stochastic inputs. Fundamentally, this approach relies on a "large" number of random samples. The precise number of iterations varies in relation to the model complexity; however, in practice, 
additional simulations are usually added until the resultant distribution does not significantly vary with added iterations. Further technical details of MCS are fully described in several texts, including Tan et al. (2007), Thomopoulos (2012), and Mooney (1997).

While business and financial applications have seen the most widespread use of MCS, mineral and energy industry practitioners have also experimented with its use, particularly in hydrocarbon reservoir analysis, mineral reserve estimation, equipment reliability, equipment scheduling, and, to some extent, process circuit design (Montenegro et al. 2013a, 2013b, 2015). The latter case, in particular, has leveraged known process models as well as known input parameter distributions to analyze the variability of potential outcomes as well as the robustness of particular circuit designs.

\subsubsection{Taguchi's Method}

Taguchi's method, as an "experimental design" protocol, has been widely utilized in statistical tolerancing for manufactured parts. Originally, Taguchi (1978) introduced this experimental design methodology as an alternative to the typical MCS approach, and then demonstrated its application in predicting the output voltage variability of a power circuit with a known response function. Nevertheless, it is mistakenly believed that Taguchi's method can be used only in the experimental design problems in which the response function is unknown (D'Errico and Zaino 1988). Similar to the MCS, Taguchi's method can be employed to predict the statistical distribution of a model result given stochastic input parameters; however, Taguchi's method notably does not require a large number of randomly sampled input parameters. Instead, Taguchi's method relies on a limited number of carefully selected input parameters and an additional analytical step to process the resultant data. The method greatly reduces the number of function evaluations and ultimately produces the moments of the final statistical distribution, which in most practical cases are just as useful as the full distribution produced by MCS. Unlike MCS, Taguchi's method can be readily implemented using a hand calculator (cf., sophisticated spreadsheet routines and/or dedicated programs for MCS) (Taguchi 1978). Furthermore, Taguchi's method is sufficiently robust to accommodate most functional forms, even non-linear examples. To execute the method, only the model results are needed; the model itself need not to be analytically defined. 
To implement Taguchi's method, one must first identify the model variables which are subject to stochastic variation and the model that produces the desired output value. Using the known mean and standard deviation of the input parameters, three-factor "levels" are defined (high, medium, and low). These factor levels are then combined with factor levels from the other stochastic parameters in full factorial. Each factorial combination produces an "experimental run" which is then calculated using the known process model. This varies from the traditional use of Taguchi's method where experimental runs are conducted to determine the process model. The results from all experimental runs are then compiled using specific formulae in order to determine the descriptive statistics (mean, standard deviation, skewness, and kurtosis) for the resulting distribution.

D'Errico and Zaino (1988) have described two versions of the Taguchi method. Both the standard (Taguchi 1978) and modified methods require similar calculations, and only slight mathematical differences separate the two. D'Errico and Zaino (1988) have described the contrasts and concluded that the modified method is superior. The calculation intensity is nearly identical to the standard method; however, the modified method has the ability to more accurately predict the higher moments of the resultant distribution. According to modified procedure, the three input factor levels $\left(\mathrm{X}_{1}, \mathrm{X}_{2}\right.$, and $\left.\mathrm{X}_{3}\right)$ are determined by:

$$
\begin{aligned}
& X_{1}=\mu \\
& X_{2}=\mu+\sigma \sqrt{3} \\
& X_{3}=\mu-\sigma \sqrt{3}
\end{aligned}
$$

where $\mu$ is the mean and $\sigma$ is the standard deviation of the input parameter.

Each experimental run is formulated from the full factorial combination of different stochastic parameters at different levels. Therefore, a model with $\mathrm{N}$ stochastic parameters will require $3^{\mathrm{N}}$ experimental runs. In the modified method (D'Errico and Zaino 1988), each experimental run is also assigned a point weight $\left(\mathrm{w}_{\mathrm{i}}\right)$ that is derived from the product of the input parameter point weights. The three different parameter levels are assigned weights of $1 / 6,4 / 6$, and $1 / 6$ for the low, middle, and high levels, respectively. After evaluating all experimental runs, the first moment $\left(\mathrm{m}_{1}\right)$ of the resultant distribution is determined by: 


$$
m_{1}=\sum_{i=1}^{N} w_{i} Y_{i}
$$

where $Y_{i}$ is the model result for run $i$, and $w_{i}$ is the total weight for model run $i$, as determined by product of the weight from each input parameter. Subsequent moments $\left(\mathrm{m}_{2}-\mathrm{m}_{4}\right)$ are calculated by:

$$
m_{k}=\sum_{i=1}^{N} w_{i}\left(Y_{i}-m_{1}\right)^{k}
$$

The standard deviation, skewness, and kurtosis can finally be calculated from the first four moments. These values represent the distribution of expected outcomes given the stochastic inputs to the original process model. The standard Taguchi method is applied identically, except, each point is given a constant unit weight (1.0), and the parameter levels are determined by a factor of $\sqrt{ } 1.5$ rather than $\sqrt{ } 3$.

\subsection{Application Example in Flotation Scale-Up}

The following example demonstrates a simple application of Taguchi's method in flotation scale-up. In this application, recovery from a perfectly-mixed industrial flotation cell is predicted from laboratory data using a first-order kinetic rate model. For simplicity, no scaling factors were applied to the rate constants, and the only difference between the laboratory and industrial cell are the choice of model type (plug flow vs. perfectly mixed). A standard two-parameter, plug-flow flotation rate model was used to fit the laboratory data:

$$
R_{\text {PlugFlow }}=R_{\infty}[1-\exp (-k t)]
$$

where $\mathrm{R}$ is the component recovery at flotation time $\mathrm{t}$; is the kinetic coefficient, and $\mathrm{R}_{\infty}$ is the maximum recovery of floatable material. The parameters, $k$ and $R_{\infty}$ are often determined from known recovery-time data using standard curve fitting techniques. 
Table 6-1: Input parameters in flotation scale-up example.

\begin{tabular}{|l|l|l|}
\hline Parameter & Mean & Standard Deviation \\
\hline$k$ & 1.617 & 0.0734 \\
\hline$R_{\infty}$ & 94.7 & 0.511 \\
\hline
\end{tabular}

Table 6-2: Experimental runs and calculations in flotation scale-up example.

\begin{tabular}{|l|l|l|l|l|}
\hline Exp. Run & $k(1 / \mathrm{min})$ & $R_{\infty}$ & Point Weight & $\begin{array}{l}\mathrm{R}_{\text {Mixed }} \\
(\mathrm{t}=2 \text { min. })\end{array}$ \\
\hline 1 & $1.490(-1)$ & $93.8(-1)$ & 0.028 & 70.24 \\
\hline 2 & $1.617(0)$ & $93.8(-1)$ & 0.111 & 71.66 \\
\hline 3 & $1.744(+1)$ & $93.8(-1)$ & 0.028 & 72.91 \\
\hline 4 & $1.490(-1)$ & $94.7(0)$ & 0.111 & 70.90 \\
\hline 5 & $1.617(0)$ & $94.7(0)$ & 0.444 & 72.33 \\
\hline 6 & $1.744(+1)$ & $94.7(0)$ & 0.111 & 73.60 \\
\hline 7 & $1.490(-1)$ & $95.6(+1)$ & 0.028 & 71.57 \\
\hline 8 & $1.617(0)$ & $95.6(+1)$ & 0.111 & 73.01 \\
\hline 9 & $1.744(+1)$ & $95.6(+1)$ & 0.028 & 74.29 \\
\hline
\end{tabular}

Similarly, plant performance can be described by the same parameters using a perfectly mixed reactor model:

$$
R_{\text {Mived }}=R_{\infty}\left[\frac{k t}{1+k t}\right]
$$

In this example, replicate batch flotation tests have produced a mean and standard deviation for the laboratory data (Table 6-1), and the circuit designer wishes to know the distribution of possible outcomes for plant recovery in the industrial cell. To apply Taguchi's Method, the high $(+1)$, medium $(0)$, and low $(-1)$ values for both parameters were calculated using Eq. 22 and combined in full factorial to produce nine experimental runs. Table 6-2 shows the inputs, point weights, and model predictions for each experimental run. The 
Table 6-3: Taguchi's method results (compared to Monte Carlo simulation).

\begin{tabular}{|l|l|l|}
\hline Parameter & $\begin{array}{l}\text { Value from Taguchi Method } \\
(\mathrm{N}=9)\end{array}$ & $\begin{array}{l}\text { Value form Monte Carlo Simulation } \\
(\mathrm{N}=250,000)\end{array}$ \\
\hline Mean & 72.307 & 72.307 \\
\hline St. Dev. & 0.872 & 0.872 \\
\hline Skewness & -0.137 & -0.142 \\
\hline Kurtosis & 3.005 & 3.059 \\
\hline
\end{tabular}

model result (plant recovery) was determined using the perfectly mixed reactor model (Eq. (5)), assuming a residence time of two minutes.

Given the model results and point weights in Table 6-2, the distribution moments were determined using Eqs. 23 and 24. Descriptive statistical parameters (mean, standard deviation, skewness and kurtosis) were lastly determined from the four distribution moments. These statistics (Table 6-3) show that the mean recovery is 72.31 with an absolute standard deviation of 0.87 . The skewness parameter indicates that the distribution is predominantly normal with only a slight negative skew.

To validate this Taguchi analysis, a MCS was also performed using the aforementioned input statistics and model parameters. This simulation included 250,000 solution iterations. Figure 6-1 shows a rolling average of each incremental solution averaged with each of its predecessors. As shown in the graph, this particular model requires at least 100,000 function evaluations before stable solution is found to two decimal places (cf., nine function evaluations for Taguchi's method). While the calculation time for this particular model is trivial in either case, the distinction becomes more prevalent as the model complexity is increased. For full flotation systems, the requisite solution iterations required by MCS would be substantially prohibitive, especially if the circuit designer wants to assess the model sensitivity for a range of potential input factors.

Furthermore, the statistical results of the MCS show exceptional similarity to the results determined from the Taguchi Method (Table 6-3). As shown, neither solution methodology produces materially different results. This similarity strongly supports the validity of the 


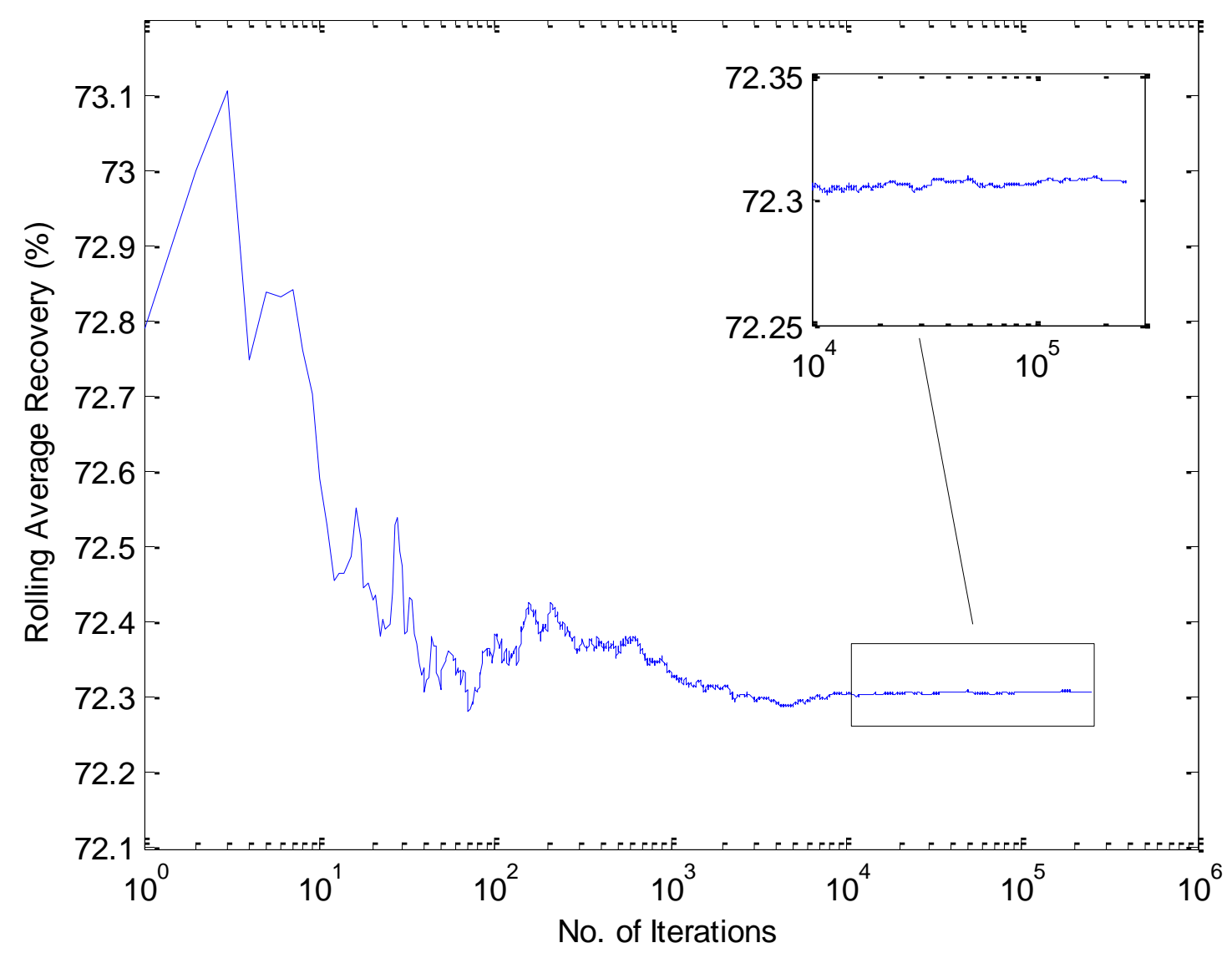

Figure 6-1: Rolling average results for Monte Carlo Simulation.

Taguchi method as a rigorous uncertainly analysis tool for cases where input variables follow a normal statistical distribution.

\subsection{Application Example in Separation Circuit Design}

As a more complex example, Taguchi's method is applied here to assess uncertainty propagation in separation circuit design. As described by Amini and Noble (2017), different circuit designs (e.g. roughing vs. scavenging) can inhibit or expand unit uncertainty depending on the recovery values and the stream connections. In this case, the circuit designer wishes to know the potential variation in circuit recovery (or mass flow rate) when given statistical information (mean and standard deviation) on unit recovery in each 


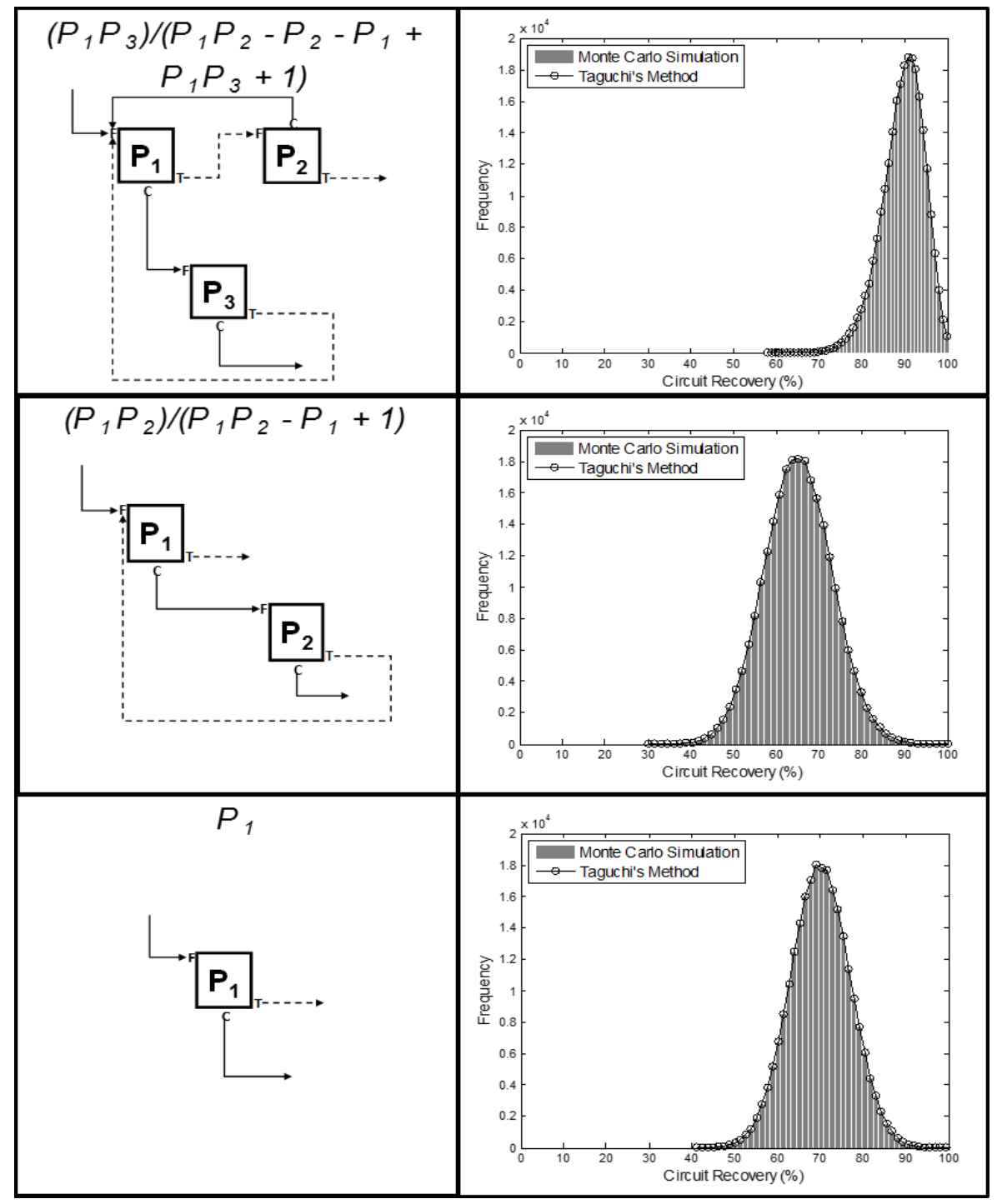

Figure 6-2: Applications of Taguchi's method in the circuit design process to identify the statistical distribution of global recovery.

individual unit. The results are to be compared to a number of different circuit designs to determine which design maximizes process performance while mitigated uncertainty. For simplicity, only three circuits are considered in this example (Figure 6-2).

As described by Noble and Luttrell (2014), the circuit recovery may be expressed as a function of the individual unit recovery values using formulae derived from linear circuit 
analysis. These formulae have been derived for the three circuits in question and are shown with the circuits in Figure 6-2. These functions represent the process model to be evaluated for each experimental run.

The recovery data for each process unit are as follows: $(\mathrm{P} 1)$ rougher recovery, mean $=$ $70 \%$, standard deviation $=7.0 \%$; $(\mathrm{P} 2)$ scavenger recovery, mean $=80 \%$, standard deviation $=8.0 \%$; $(\mathrm{P} 3)$ cleaner recovery, mean $=75 \%$, standard deviation $=7.5 \%$. In practice, these values may be determined from historical data or derived from a known process model. Next, the low, medium, and high values of unit recovery were determined for each input unit (Eq. 22), and then these values were combined in full factorial to construct 3, 9, and 27 experimental runs for one, two, and three-unit circuits, respectively. Each experimental run was evaluated using the circuit analytical formulation, and lastly, the distribution moments were evaluated using Eqs. 23 and 24

The results of this procedure are showed alongside similar MCS results in Figure 6-2. Similar to the previous example, the Taguchi's method outcomes coincide very well with MCS validating the utility of the method in preliminary uncertainty evaluation of separation circuits. Furthermore, these results confirm those of Amini and Noble (2017) showing that the balanced rougher-scavenger-cleaner circuit reduces uncertainty when compared to the alternative designs.

\subsection{Summary and conclusions}

This chapter has introduced Taguchi's method, as a design of experiment strategy, to quantify uncertainty propagation in separation circuit design problems. The application of Taguchi's method was demonstrated in a flotation scale-up problem and a circuit design problem procedure. In both cases, Taguchi's method produced results identical to that of MCS, while requiring much fewer simulations, often several orders of magnitude lower. In future works, Taguchi's method will be combined with global sensitivity analysis to provide rigorous procedure to determine the level of uncertainty and to identify the most influential unit in these uncertainty propagations. 


\subsection{Bibliography}

Amini SH, Noble A. Application of linear circuit analysis in evaluation of mineral processing circuit design under uncertainty. Minerals Engineering. Elsevier; 2017;102:18-29.

D'Errico JR, Zaino NA. Statistical tolerancing using a modification of Taguchi's method. Technometrics. Taylor $\backslash \&$ Francis; 1988;30(4):397-405.

Ghaffari A, Hayati M, Shekholeslami A. Probability and Sensitivity Analysis in Flotation Circuit of Bama Lead and Zinc Processing Plant Using Monte Carlo Simulation Method. Mineral Processing and Extractive Metallurgy Review. Taylor \& Francis; 2012;33(6):416-26.

Kraslawski A. Review of applications of various types of uncertainty in chemical engineering. Chemical Engineering and Processing: Process Intensification. Elsevier; 1989;26(3):185-91.

Montenegro MR, Bruckard WJ, Gálvez ED, Cisternas LA. Arsenic-rejection flotation circuit design and selection based on a multiple-objective evaluation. Minerals Engineering. Elsevier; 2013 a;45:22-31.

Montenegro MR, Gálvez ED, Cisternas LA. The effects of stage recovery uncertainty in the performance of concentration circuits. International Journal of Mineral Processing. Elsevier; 2015;143:12-7.

Montenegro MR, Sepúlveda FD, Gálvez ED, Cisternas LA. Methodology for process analysis and design with multiple objectives under uncertainty: Application to flotation circuits. International Journal of Mineral Processing. Elsevier; 2013 b;118:15-27.

Mooney CZ. Monte carlo simulation. Sage Publications; 1997.

Noble A, Luttrell GH. The matrix reduction algorithm for solving separation circuits. Minerals Engineering. Elsevier; 2014;64:97-108.

Taguchi G. Performance analysis design. The International Journal of Production Research. Taylor \& Francis; 1978;16(6):521-30. 
Tan RR, Foo DC, Manan ZA. Assessing the sensitivity of water networks to noisy mass loads using Monte Carlo simulation. Computers $1 \&$ chemical engineering. Elsevier; 2007;31(10):1355-63.

Thomopoulos NT. Essentials of Monte Carlo simulation: Statistical methods for building simulation models. Springer Science \& Business Media; 2012.

Xiao Z, Vien A. Limitations of variance analysis using propagation of variance. Minerals engineering. Elsevier; 2003;16(5):455-62. 


\title{
Chapter 7
}

\section{A Techno-Economic Strategy for the Optimal Design of Cell-Based Flotation Circuits under Uncertainty}

\begin{abstract}
(ABSTRACT)
Many circuit design and optimization strategies rely on deterministic modeling approaches that use static values for the technical and economic input parameters. Unfortunately, for mineral processing operations, these input parameters (e.g. feed grade, kinetic coefficients, and mineral price) and scale-up models may be subject to various levels of uncertainty. This uncertainty often leads to ambiguity in the design and optimization process, as certain solutions may only be optimal under specific input scenarios. One promising strategy to address this shortcoming is through Sample Average Approximation (SAA) methodology, a stochastic approach to handling uncertainty that has been widely applied in other disciplines such as supply chain and facility location management problems. In this study, a technoeconomic optimization methodology is formulated for the optimal design of cell-based flotation circuits. Next, the SAA approach is implemented in the original optimization formulation to consider the stochasticity of input parameters. The developed optimization approach may be used to determine the optimal size and number of flotation cells with the objective of maximizing net present value. Finally, an application example is provided to properly demonstrate the capability of this novel technique for selecting the optimal and/or series of potentially optimal circuit solutions.
\end{abstract}

Keywords: circuit design; flotation; stochastic optimization; sample average approximation. 


\subsection{Introduction}

\subsubsection{Background}

Flotation is unquestionably the most prominent separation technology that is widely employed for treating verity of metallic, nonmetallic, and fuel minerals (Fuerstenau and Han 2003; Fuerstenau et al. 2007; Wills 2011). In conventional froth flotation, the separation performance of single-stage circuit is often insufficient to produce a high-grade concentrate at the acceptable level of recovery. To overcome this inherent deficiency and achieve an effective separation, multi-stage separation circuit configurations are employed. The separation performance of these complicated separation circuits are influenced by several factors, including feed composition, circuit design layout, dimension and number of flotation cells, and obviously, physical and chemical-based operational parameters (Yingling 1993; Wills 2011; Amini and Noble 2017). In addition to the circuit metallurgical performance, profitability of the final system is a fundamental goal of the flotation circuit design procedure. Finding the optimal flotation circuit configuration, as a multi-objective optimization problem, is a challenging task.

As fully discussed in chapter 2 , over the last 40 years, several strategies were implemented for the optimal design of flotation circuits based on the technical and/or financial objectives. Given the opposite trends between the grade and recovery factors, several researchers have attempt to find the flotation circuit with the optimal levels of global recovery and concentrate grade (Green 1984; Reuter et al. 1988; Reuter and Van Deventer 1990; Guria et al. 2005a, 2005b, 2006). Nevertheless, as circuit economics (e.g. operating and capital cost as well as revenue) and associated technical consequences can be collectively formulated in a decision procedure, several researchers have developed financial-based optimization techniques for determining the optimal or sub-optimal circuit solutions. While Abu-Ali and Sabour (2003) have specified the cost minimization as an objective function of the optimization strategy, Cisternas et al. $(2005,2006)$ and Schena et al. $(1996,1997)$ have developed profit-based optimization methodologies.

Most of the available mathematical models (e.g. (Schena et al. 1996, 1997; Cisternas et al. 2005, 2006)) generate a mixed integer non-linear formulation to select the optimal, or 
sub-optimal, superstructure after comparing the techno-economic outcomes of numerous distinct designs. Guria et al. (2005a, 2005b, 2006) and Ghobadi et al. (2011) have utilized genetic algorithms to solve similar cell-based flotation circuit design problem. Most, if not all, of these studies have employed first-order kinetic models considering similar kinetic coefficients for mineral species in the individual flotation cells. Despite the oversimplification of the model, the similar strategy is followed in industrial practices (Guria et al. 2005a).

Despite the advancement in the flotation circuit design process, very few studies consider the inherent uncertainty associated with various technical and financial factors, including price, feed composition, and kinetic coefficient. Recently, Jamett et al. (2015) addressed the metal price and ore grade uncertainty via a stochastic flotation circuit design technique. In the original problem, the size of cells in each flotation stage was considered as known parameters while operational factors incorporated as variables. They utilized various local and global optimization solver to determine the optimal circuit solution. Given the high complexity of applied optimization routine, these researchers only considered nine individual scenarios using three levels of ore grade and metal price. This study concluded that the stochastic optimization approach can be a beneficial tool in the optimal design of mineral processing circuits. However, the authors suggested that the obtained circuit solutions must be carefully validated through further simulations and expert analysis.

\subsection{Problem statement and objectives}

As a supplement to the available circuit optimization techniques, this chapter provides a novel techno-economic strategy for determining the optimal number and size of flotation cells with the objective of maximizing circuit net present value. Additionally, a unique stochastic optimization methodology is developed to address the uncertainty of various input parameters. In summary, the following objectives are identified:

- Develop a deterministic model to maximize the NPV of the flotation system while satisfying the grade requirements dictated by downstream smelters; 
- Investigate the sensitivity of the model objective function to inherent uncertainties associated within metal price, feed composition, and kinetic coefficient;

- Construct a stochastic optimization routine using the principles of SAA approach for finding a series of optimal flotation circuit design under uncertainty;

- Apply Taguchi's method to the deterministic model to determine the NPV of the circuit design when uncertainty of input parameters is incorporated;

\subsection{Deterministic formulation of circuit design}

In this section, a techno-economic strategy for finding the optimal separation circuit configuration, based on the deterministic platform, is provided. Table 7-1 lists the symbols used to describe the problem and categorized them into sets and indices, variables, certain constants, and potentially uncertain constants. The remaining of this section describes the distinct parts of the circuit design deterministic strategy.

\subsubsection{Optimal design algorithm}

Various technical (e.g. recovery or grade maximization) or financial (e.g. revenue maximization or cost minimization) objective functions can be assigned for the flotation circuit design optimization problem. However, the importance of total life cycle cost analysis necessities the use of net present value (NPV), as the objective of the optimization problem. $N P V$ accurately considers not only the lifetime operation and maintenance costs but also the initial investments. Therefore, the objective of the deterministic formulation seeks to determine the optimal number $(N)$ and size $(V)$ of cells for each flotation stages to maximize the $N P V$ of the project. The $N P V$ of separation circuit design (i.e. objective function) may be adequately expressed in terms of revenue (Rev), operating cost (Opex), capital cost (Capex), discount rate (DRate), and life of the project (Life), as follows:

$$
\text { Max. }(\text { Rev-Opex }) \times\left(\frac{1-(1+\text { DRate })^{- \text {Life }}}{\text { DRate }}\right)-\text { Capex }
$$


Table 7-1: Description of symbols used for sets, indices, variables, certain constants, and potentially uncertain constants.

\begin{tabular}{|c|c|c|c|}
\hline \multicolumn{4}{|c|}{ Nomenclature } \\
\hline \multicolumn{2}{|c|}{ Sets \& indices } & \multicolumn{2}{|c|}{ Certain constants } \\
\hline$i$ & number of flotation stages & DRate & discount rate \\
\hline$j$ & number of circuit streams & Life & life of the project \\
\hline \multicolumn{2}{|c|}{ Variables } & $F P$ & fraction of metal paid \\
\hline $\operatorname{Rev}$ & revenue & $u$ & grade deductions \\
\hline Opex & operating cost & $R f c$ & refinery charges \\
\hline Capex & capital cost & $\operatorname{Trc}$ & treatment charges \\
\hline$Y_{C}$ & mass yield to concentrate stream & $T_{F}$ & feed mass flow rate \\
\hline$G_{C}$ & grade of valuable mineral in concentrate stream & $T_{O, F}$ & ore mass flow rate in feed stram \\
\hline$N_{i}$ & number of cells in ith flotation stage & Energy & energy cost \\
\hline$V_{i}$ & size of cells in ith flotation stage & Days & number of working days per year \\
\hline$T_{C}$ & solid flow rate in the concentrate stream & $\mathrm{OpH}$ & power cost factor $\mathrm{kWh}$ per $\mathrm{m} 3$ of the cell size \\
\hline$T_{O, C}$ & ore flow rate in the concentrate stream & $h$ & constant \\
\hline$T_{S, j}$ & mass flow rate of mineral $\mathrm{S}$ in jth stream & CapA & constant \\
\hline$T_{j}$ & total mass flow rate in jth stream & CapB & constant \\
\hline$P_{S, i}$ & recovery of mineral $\mathrm{S}$ in the $i^{\text {th }}$ stage & $r_{m e t / m i n}$ & mass ratio of the pure metal in the mineral \\
\hline$\tau_{i}$ & retention time in ith flotation bank & $S G$ & solid density \\
\hline \multirow{5}{*}{$T_{i}$} & \multirow{5}{*}{$\begin{array}{l}\text { feed mass of } i^{\text {th }} \text { flotation stage } \\
\text { preliminary retention time in ith flotation bank }\end{array}$} & $X_{i}$ & solid concentration in $\mathrm{i}^{\text {th }}$ flotation stage \\
\hline & & \multicolumn{2}{|c|}{ Potential uncertain constants } \\
\hline & & $q$ & metal price \\
\hline & & $K_{S, i}$ & kinetic coefficient for mineral $S$ in the $\mathrm{i}^{\text {th }}$ unit \\
\hline & & & feed grade \\
\hline
\end{tabular}

where discount rate and the life of project are explicitly specified; while other financial variables in the objective function (i.e. Rev, Opex, Capex) must be accurately determined. The maximization of the proposed objective function is achieved through the maximization of revenue and minimization of associated capital and operating costs. The following subsections describe the calculation of these flotation economic parameters along with flotation metallurgical factors. 


\subsubsection{Modeling of flotation economics}

According to Schena et al. (1996), the annual revenue of a flotation plant can be derived as the function of both technical parameters (e.g. yield and concentrate grade) as well as financial factors (e.g. price and downstream costs). For the base metals flotation plants, the following expression may be employed to estimate the gained revenue from concentrate sales:

$$
\operatorname{Rev}=T_{F} \times\left(Y_{C} \times F P \times\left(G_{C}-u\right) \times(q-R f c)-Y_{C} \times \operatorname{Tr} c\right)
$$

where $T_{F}$ and $Y_{C}$ are the hourly flow rate of the plant feed and concentrate stream, $G_{C}$ is the final concentrate grade, and $q$ is the metal quotation on the commodities market. Furthermore, downstream smelters apply further charges, including refinery cost $(R f c)$, treatment cost $(\operatorname{Tr} c)$, grade deduction $(u)$, and fraction of metal paid $(F P)$, to produce a saleable metal. Treatment charges are imposed based on the concentrate tonnage $\left(Y_{C}\right)$ while the refinery charges are defined according to a metal content $\left(G_{C}\right)$. On the other hand, the grade deduction and the fraction of metal paid depend on the recovery efficiency of the smelter. While not visible in Eq. 28, the expected annual revenue of the plant depends on the size and number of flotation cells as operational factors (e.g. $Y_{C}, G_{C}$ ) are indirectly estimated in terms of cell size and number. Following sub-section describes in detail the relationship between these parameters.

The operating cost is estimated by the broad assumption that power cost is $40 \%$ of the total operating cost (the other 60\% include labor, reagents, maintenance, etc.) (Abu-Ali and Sabour 2003). Power cost is calculated directly by assuming a constant cell power intensity (i.e. $\left.\mathrm{kW} / \mathrm{m}^{3}\right)$. For this study, the power intensity was assumed to be $\$ 0.07\left(\mathrm{Kw} / \mathrm{m}^{3}\right)$. Thus, power cost scales linearly with respect to cell size, and overall operating cost scales as well. Furthermore, capital cost is estimated based on an exponential cost factor ( $\operatorname{Cap} B=0.57$ ). The annual operating cost and capital cost of the cell-based flotation system can be determined as a sum of the associated costs to each flotation stages (Mular 1978; Schena et al. 1996; Abu-Ali and Sabour 2003): 


$$
\begin{aligned}
& \text { Opex }=\sum_{i} N_{i} \times\left(\frac{(24 \times \text { Energy } \times \text { Days } \times O p H)}{0.4} \times V_{i}^{1-h}\right) \\
& \text { Capex }=\sum_{i} N_{i} \times C a p A \times V_{i}^{C a p B}
\end{aligned}
$$

where $N_{i}$ and $V_{i}$ are the number and size of cells in the $i^{\text {th }}$ flotation stage. In addition to these variables, the energy cost (Energy), the number of working days per year (Days), power consumption cost per $\mathrm{m}^{3}$ of the cell volume $(O p H)$, and several constants (i.e. $h$, CapA, and CapB) are employed to calculate these economic measures. As illustrated in Eq. 29, the operating and capital costs are elevated as the size and number of flotation cells are increased.

\subsubsection{Modeling of flotation metallurgy}

The separation performance of flotation system directly defines the annual revenue as circuit yield $\left(Y_{C}\right)$ and concentrate grade $\left(G_{C}\right)$ are the only two variables in the general revenue formulation (see Eq. 28). In other words, the maximization of revenue is possible via an optimal circuit separation performance with high values of yield and concentrate grade. This section describes a novel approach for the accurate estimation of flotation technical parameters $\left(Y_{C}\right.$ and $\left.G_{C}\right)$ in terms of number $(N)$ and size $(V)$ of flotation cells.

The circuit yield, or mass recovery, and concentrate grade are both can be precisely determined in terms of mass flow rate of mineral species within feed and concentrate streams:

$$
\begin{aligned}
Y_{C} & =\frac{T_{C}}{T_{F}} \times 100 \\
G_{C} & =\frac{T_{O, C}}{T_{O, F}} \times r_{m e t / m i n} \times 100
\end{aligned}
$$

where $T_{C}$ and $T_{F}$ are the total solid flow rates in the concentrate and feed streams, $T_{O, C}$ and $T_{O, F}$ are the ore mass flow rate in the concentrate and feed streams, and $r_{\text {metrmin }}$ is the mass ratio of the pure metal in the mineral. For example, this ratio for copper associated in chalcopyrite mineral is equal to 0.346 . As shown in Eq. 30, the accurate estimation of circuit 
Table 7-2: Circuit-descriptive matrices utilized in the matric reduction algorithm, after (Noble and Luttrell 2014).

\begin{tabular}{|c|c|c|c|c|c|c|c|}
\hline & \multicolumn{3}{|l|}{$F_{\text {Matrix }}$} & \multicolumn{3}{|l|}{$\mathrm{P}_{\text {Matrix }}$} & \multirow{2}{*}{\begin{tabular}{|l} 
C $_{\text {Vector }}$ \\
Feed
\end{tabular}} \\
\hline & Stage $_{1}$ & Stage $_{2}$ & $\begin{array}{ll}\ldots & \text { Stage }_{\mathrm{i}}\end{array}$ & Stage $_{1}$ & Stage $_{2}$ & ... Stage $_{\mathrm{i}}$ & \\
\hline Stream $_{1}$ & $\mathrm{~F}_{1,1}$ & $\mathrm{~F}_{1,2}$ & $\ldots \mathrm{F}_{1, \mathrm{i}}$ & $\mathrm{P}_{1,1}$ & $\mathrm{P}_{1,2}$ & $\ldots \quad P_{1, \mathrm{i}}$ & $\mathrm{C}_{1,1}$ \\
\hline Stream $_{2}$ & $\mathrm{~F}_{2,1}$ & $\mathrm{~F}_{2,2}$ & \begin{tabular}{l|l}
$\ldots$ & $F_{2, \mathrm{i}}$ \\
\end{tabular} & $\mathrm{P}_{2,1}$ & $\mathrm{P}_{2,2}$ & \begin{tabular}{l|l}
$\ldots$ & $\mathrm{P}_{2, \mathrm{i}}$ \\
\end{tabular} & $\mathrm{C}_{2,1}$ \\
\hline$\vdots$ & $\vdots$ & $\vdots$ & \begin{tabular}{|l|l}
$\ldots$ & $\vdots$ \\
\end{tabular} & $\vdots$ & $\vdots$ & \begin{tabular}{|l|l}
$\ldots$ & $\vdots$ \\
\end{tabular} & $\vdots$ \\
\hline Stream $_{\mathrm{j}}$ & $\mathrm{F}_{\mathrm{j}, 1}$ & $\mathrm{~F}_{\mathrm{i}, 2}$ & \begin{tabular}{|l|l}
$\ldots$ & $\mathrm{F}_{\mathrm{i}, \mathrm{i}}$ \\
\end{tabular} & $\mathrm{P}_{\mathrm{i}, 1}$ & $\mathrm{P}_{\mathrm{i}, 2}$ & \begin{tabular}{l|l}
$\ldots$ & $\mathrm{P}_{\mathrm{i}, \mathrm{i}}$ \\
\end{tabular} & $C_{i, 1}$ \\
\hline
\end{tabular}

performance indicators requires the precise determination of the mass flow rate of various mineral species within circuit streams.

According to Noble and Luttrell (2014), the "matrix reduction algorithm", as an efficient and accurate tool, can be utilized to estimate the mass flow rate of mineral species within the separation circuit streams. The algorithm relies on three circuit-descriptive matrices (i.e. the feed and product matrices as well as the initial condition vector) and a single governing equation to solve the final circuit stream solution. Table 7-2 shows the dimension and elements of these matrices. As a note, in the matrix reduction algorithm, junction units are considered as individual stages.

The $F_{\text {Matrix }}$ specifies whether $j^{\text {th }}$ stream provides feed for the $i^{\text {th }}$ stage or not. The value of $F_{j, i}$ is 1 if stage $i$ is fed by stream $j$ while the zero value of $F_{j, i}$ indicates that stream $j$ does not feed unit $i$. Similarly, in constructing $P_{\text {Matrix }}$, the relationship between stream $j$ and unit $i$ is investigated. Elements of this matrix may vary between 0 , indicating no relation between stream $\mathrm{j}$ and stage $i$, and 1 , specifying that the entire product of the stage $i$ (junction unit) reports into stream $j$. The values of $P_{\text {Matrix }}$ elements determine the probability that the distinct class of species, which feed into unit $i$, report to stream $j$. It is worth to note that each specific class of particles (e.g. ore or gangue) requires a unique product matrix $\left(P_{\text {Matrix }}\right)$ using the numerical values of unit recovery for that particle class. Furthermore, the initial condition vector $\left(C_{\text {Vector }}\right)$ demonstrates the original circuit feed stream(s) and initializes the mass flow rate of different mineral species in the feed stream. As such, a distinct mineral has a unique 
$C_{\text {Vector }}$. If stream $\mathrm{j}$ is a circuit feed stream, the value of the feed mass flow rate is assigned to the grade (mass) of mineral in the feed stream $\left(G_{F}\right)$. All other elements are assigned a value of zero.

After construction of these fundamental matrices, the following equation may be used to estimate the mass flow rate of each mineral species in all separation circuit streams $\left(T_{s_{j}}\right)$, thereby calculating the total mass flow rate in each stream $\left(T_{i}\right)$ :

$$
\begin{aligned}
& T_{S, j}=\left(I_{j, i}-P_{\text {Matrix }, S} \times F_{\text {Matrix }, S}^{\prime}\right)^{-1} \times C_{\text {Vector }} . \\
& T_{j}=\sum_{S} T_{S, j}
\end{aligned} .
$$

In the current optimization formulation, the recovery of individual mineral species within each flotation stage is considered variable to be determined. As a result, the following equation, based on the first-order rate equation for a series of perfectly-mixed cells, may be employed to adequately determine the recovery of each particle class in the $i^{\text {th }}$ stage $\left(P_{S, i}\right)$ :

$$
P_{S, i}=1-\left(1-\frac{K_{S, i} \tau_{i}}{1+K_{S, i} \tau_{i}}\right)^{N}
$$

where $K_{S, i}$ is the kinetic coefficient constant for the particle class of $S$ in the $\mathrm{i}^{\text {th }}$ unit, and $\tau_{i}$ is the retention time in each flotation bank of $N$ cells. $K_{S, i}$ is typically estimated using a systematic lock-cycle and pilot scale experimentation. One of the most important parameters in sizing a flotation cell and improving the circuit performance is retention time. The mean retention time can be expressed as the ratio of active cell volume to the cell volumetric flow rate utilizing the following formulation:

$$
\tau_{i}=60 \times\left(\frac{V_{i}}{\frac{T_{i}}{S G}+\frac{T_{i}}{X_{i}}-T_{i}}\right)
$$


where $T_{i}$ is the feed mass of $\mathrm{i}^{\text {th }}$ individual stage, $S G$ is the solid density, and $X_{i}$ is the solid concentration in $\mathrm{i}^{\text {th }}$ stage. As illustrated in Eqs. 32 and 33, the unit recovery and retention time factors are dependent. In other words, to accurately identify the optimal values of retention time in individual flotation stages, the proper values of recoveries are required, and vice versa. These values are typically solved via iterative calculations, a challenging task in optimization models. To appropriately estimate these variables, a novel approach is implemented in which the optimization problem is initiated employing a set of preliminary retention time values $\left(\tau_{i, P}\right)$ in each flotation bank. These values are then applied in Eq. 32 to calculate the unit recovery values. In the next phase, the calculated recovery values are employed to determine the secondary values for the retention times associated with each flotation bank. Now, the calculated values of retention time replace the preliminary retention time values and this procedure is repeated until the following constraint is satisfied. It worth to note that the following constraint is evaluated concurrently with the optimization problem.

$$
\left(\tau_{i}-\tau_{i, P}\right)^{2} \leq \varepsilon
$$

$\varepsilon$ may be considered as a very small number. Following the results of these phases, the circuit mass yield and the final concentrate grade are determined. Figure 7-1 depict the explained procedure for the accurate estimation of technical parameters in terms of number and size of flotation cells.

\subsubsection{Arbitrary constraints}

Overall, the proposed techno-economic formulation for determining the optimum number and size of cell in the flotation system is placed into the category of non-convex mixed integer non-linear program class. Typically, various methodologies and optimization solvers may be utilized to properly solve these types of mathematical models. However, given the high complexity of this optimization model, majority of available techniques are computationally expensive. As such, following constraints are suggested to be added into the original strategy for the effective reduction in the size of the model. 


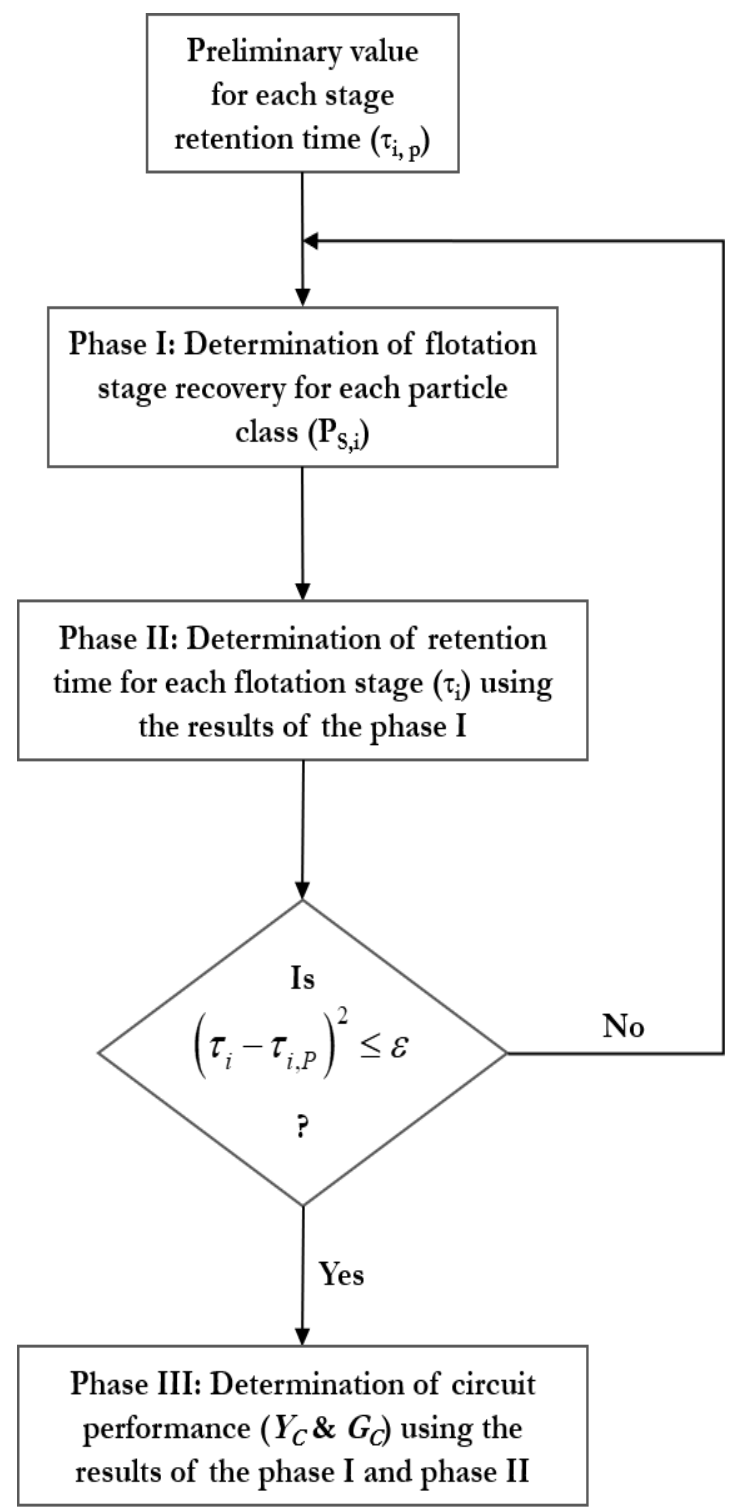

Figure 7-1: Symbolic diagram of the decision procedure for the determination of the flotation performance indicators (e.g. yield and concentrate grade).

- Constraints on the size $(V)$ and number (N) of flotation cells: These constraints can be added to the general formulation according to the available cell sizes in the industry and typical number of cells in an individual flotation bank. Based on these observation, the following constraints are imposed: 


$$
\begin{aligned}
& N_{\text {min }} \leq N_{i} \leq N_{\text {max }} \\
& V_{\text {min }} \leq V_{i} \leq V_{\text {max }}
\end{aligned}
$$

- Constraints on the concentrate grade $\left(G_{C}\right)$ : This constraint is mainly dictated by downstream smelters considering the technological limitation of the smelting process. The following concentrate grade constraint indirectly limits the mass yield projected by the flotation system:

$$
G_{C, \min } \leq G_{C}
$$

Based on complexity of the given flotation circuit configuration, several constraints on the stage recovery and retention time values may be added to the original formulation.

\subsection{Stochastic optimization of circuit design}

As explained in the introduction section, several techno-economic parameters, which are involved in the separation circuit design and operation, may subject to different degrees of uncertainties. In the current study, metal price, feed grade, and kinetic coefficient are identified as the potentially uncertain constants (Table 7-1). While the uncertainty associated with the metal price is completely controlled by the commodity market, the heterogeneity of run-of-mine, operation parameters' variability, and experimental as well as measurement errors may result in generating various degrees of uncertainty in the feed grade and kinetic coefficient factors. In this section, a systematic sensitivity analysis is first carried out on these three parameters to investigate how the uncertainty of these factors may alter the project $N P V$ and the circuit design solutions. Following the findings of this part of the study, Sample Average Approximation (SAA) technique is applied to construct a stochastic circuit optimization platform. Finally, the results of this novel stochastic optimization approach are compared against the data produced via the application of Taguchi's method in the optimal design of cell-based flotation system. 


\subsubsection{Sensitivity analysis of uncertain parameters}

The addition of input parameter uncertainty grossly elevates the complexity of the proposed MINLP model, and this complexity is further elevated with each additional uncertain input factor. As a result, the number of uncertain factors should be minimized by a rigorous preliminary assessment of the model sensitivity. The optimal circuit solution is separately determined at three levels of each uncertain parameters and then the significance of each parameter's uncertainty is investigated. The importance of each factor in identifying the final circuit solution indicate whether the parameter must be considered uncertain in the final stochastic formulation or not.

\subsubsection{Sample average approximation approach}

The sample average approximation (SAA) methodology is a common technique for solving large-scale optimization problem by the means of Monte Carlo simulation (MCS). In this technique, a set of random numbers, representing the uncertain parameter, is generated and then the expected value of objective function is determined utilizing a sample average approximation derived from these random numbers. The ultimate stochastic problem is then solved using a routine deterministic optimization technique. The process is replicated several times employing different sets of random numbers; and finally, the optimal candidate solutions are gathered and compared against one another. Several authors have extensively review the principles of SAA method along with its implementation in different areas of stochastic optimization (Mak et al. 1999; Kleywegt et al. 2002; Verweij et al. 2003; Bardossy and Raghavan 2017).

For the simple illustration of the SAA approach, the following optimization problem formulation is considered:

$$
\min _{x \in S}\left\{g(x):=\mathrm{E}_{P} G(x, W)\right\}
$$


where $W$ (uncertain constant) is a set of random numbers with a probability distribution of $P, S$ is a finite number of set, $G(x, W)$ is a real valued function in terms of $x$ and $W$, and $E_{P}$ $G(x, W)$ is the corresponding expected value.

Now, let $W^{1}, \ldots, W^{N}$ be an independently and identically distributed random sample of $N$ realizations of the random vector $W$. the sample average approximation form of the original formulation can be expressed as:

$$
\min _{x \in S} \hat{g}_{N}(x):=\frac{1}{N} \sum_{j=1}^{N} G\left(x, W^{j}\right)
$$

The SAA stochastic problem is then solved repeatedly using a deterministic optimization algorithm. By generating $S$ independent random number samples, with the size of $N$, and solving the associated SAA problems, the final objective function values of $\hat{g}_{N 1}\left(x_{1}\right), \hat{g}_{N 2}\left(x_{2}\right), \cdots, \hat{g}_{N R}\left(x_{R}\right)$ and candidate solutions of $\mathrm{x}_{1}, x_{2}, \cdots, x_{R}$ are obtained. Of all candidate optimal solutions, typically, the one that generates the minimum value of objective function and those with highest frequency may be selected as the final optimal solutions of the stochastic problem.

The current study has addressed the capability of SAA methodology for finding the optimal size and number of cells in the flotation system under uncertainty. According to the sensitivity analysis results, significant uncertain parameters were incorporated in the original MINLP problem as a set of random numbers with the defined probability distributions (e.g. uniform, normal, and lognormal) having the similar statistical measures. The constructed stochastic optimization problem was then solved repeatedly for different sets of random numbers and then the NPV values were estimated. The final solution for the stochastic problem was the one with the maximum NPV value and the most repeated solutions (i.e. the most robust solution). Finally, the obtained results from this phase of the study was compared against the deterministic problem circuit solution. This comparison may indicate the importance of considering uncertainty in the process of finding the optimal flotation system and show the significant of statistical distribution shapes for various uncertain parameters. 


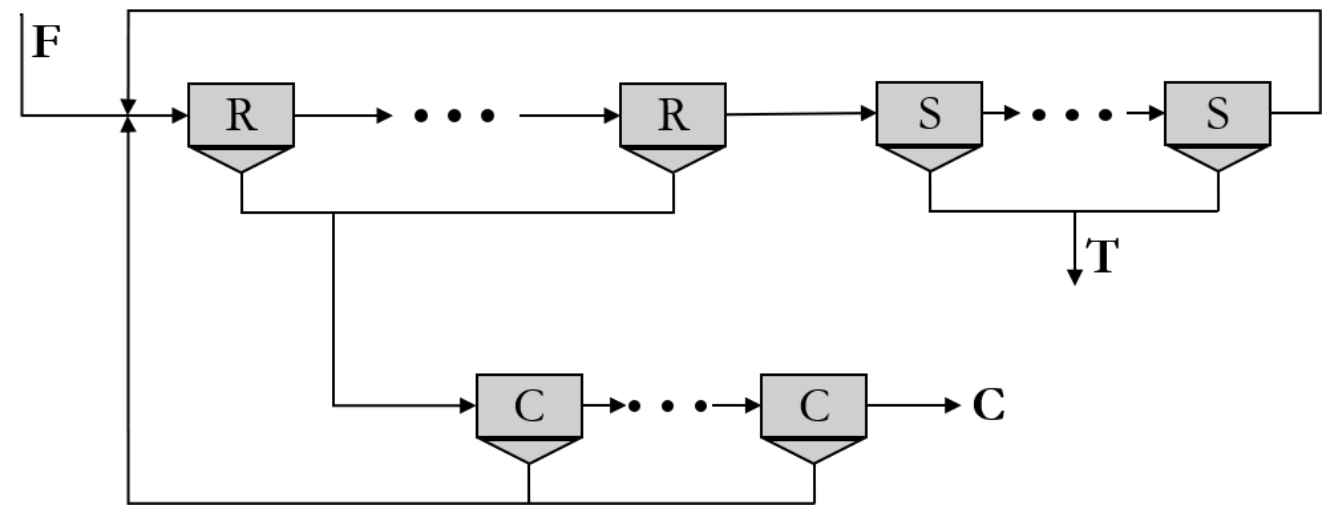

Figure 7-2: A balanced circuit used for the application example.

\subsubsection{Taguchi's method}

As an extension to the SAA approach, in the final phase of this study, the applicability of Taguchi's method in determining the optimal flotation circuit solution is investigated. As comprehensively explained in the previous chapter, Taguchi's method is an alternative tool for solving the stochastic problems. The capability of this straightforward technique was earlier verified for analyzing the circuit uncertainty projection. Following the sensitivity analysis data, the uncertain parameters are incorporated as the three carefully selected points in the optimization problem and the problem is solved deterministically. The application of Taguchi's method avoids an increase in the size of the stochastic problem when it compares with the SAA approach. The final circuit solution obtained from this method is compared with the results of deterministic and SAA methodologies.

\subsection{Application example}

\subsubsection{Problem description}

To properly demonstrate the implementation of the proposed techno-economic optimization strategy, a simple cell-based flotation circuit configuration is provided (Figure 7-2). This balanced flotation circuit with recycling concentrate and tailings streams to the 
Table 7-3: Input parameters in the deterministic circuit optimization problem.

\begin{tabular}{|c|c|c|c|}
\hline \multicolumn{2}{|c|}{ Potential uncertain constants } & \multicolumn{2}{|l|}{ Certain constants } \\
\hline$q(\$ /$ ton $)$ & 6000 & Drate (\%) & 10 \\
\hline$G_{F}(\%)$ & 0.01 & Life (year) & 25 \\
\hline$K_{O, R}(1 / \mathrm{min})$ & 0.225 & $F P$ & 0.975 \\
\hline$K_{O, S}(1 / \mathrm{min})$ & 0.2 & $u$ & 0.01 \\
\hline$K_{O, C}(1 / \mathrm{min})$ & 0.275 & $R f_{c}(\$ /$ ton $)$ & 200 \\
\hline$K_{G, R}(1 / \mathrm{min})$ & 0.005 & $\operatorname{Trc}(\$ /$ ton $)$ & 55 \\
\hline$K_{G, S}(1 / \min )$ & 0.01 & $T_{F}($ ton $/ h r)$ & 1000 \\
\hline \multirow[t]{12}{*}{$K_{G, C}(1 / \mathrm{min})$} & 0.0075 & $T_{O, F}($ ton $/ h r)$ & 10 \\
\hline & & Energy $(\$ / k W h)$ & 0.07 \\
\hline & & Days (day/year) & 340 \\
\hline & & $O p H\left(k W h / m^{3}\right.$ of cell $)$ & 2.4 \\
\hline & & $b$ & 0.04 \\
\hline & & CapA & 15422.88 \\
\hline & & CapB & 0.57 \\
\hline & & $r_{\text {mettmin }}$ & 0.346 \\
\hline & & $S G$ & 2.65 \\
\hline & & $X_{R}(\%)$ & 35.04 \\
\hline & & $X_{S}(\%)$ & 37.04 \\
\hline & & $X_{C}(\%)$ & 25.34 \\
\hline
\end{tabular}

rougher stage is designed to treat a chalcopyrite mineral. It is assumed that this flotation circuit represents one of the several alternative circuits that are selected through the preliminary circuit design process. The main purpose of this stage of the circuit design procedure is to determine the most profitable flotation circuit design candidates with the proper size and number of cells. These alternative circuit solutions may then be selected for further laboratory and pilot-scale experimentations.

Table 7-3 shows the values of different input parameters, including certain and potentially uncertain constants. The flotation circuit treats 1000 ton per hour of ore 
Table 7-4: Optimal flotation circuit solution using the deterministic approach.

\begin{tabular}{|ccc|}
\hline Objective Function & $\mathrm{NPV}(\$)$ & $1.67 \mathrm{E}+07$ \\
\hline \multirow{3}{*}{ Circuit Solution } & $N_{R}, V_{R}\left(m^{3}\right)$ & 4,194 \\
& $N_{S}, V_{S}\left(m^{3}\right)$ & 2,182 \\
& $N_{C}, V_{C}\left(m^{3}\right)$ & 2,16 \\
\hline Financial Outcomes & $\operatorname{Rev}(\$)$ & $1.90 \mathrm{E}+08$ \\
(Intermediate & Capex $(\$)$ & $1.99 \mathrm{E}+06$ \\
Calculations) & Opex $(\$)$ & $3.26 \mathrm{E}+06$ \\
\hline & $P_{O, R}, P_{G, R}(\%)$ & $93.49,8.26$ \\
Technical Outcomes & $P_{O, S}, P_{G, S}(\%)$ & $74.20,9.03$ \\
(Intermediate & $P_{O, C}, P_{G, C}(\%)$ & $65.67,3.75$ \\
Calculations) & Concentrate grade $(\%)$ & 25.15 \\
& Recovery $(\%)$ & 97.34 \\
\hline
\end{tabular}

containing $0.1 \%$ of chalcopyrite (10 ton per hour) in average. The downstream smelter is specified the minimum concentrate grade of $25 \%$. The maximum and minimum available flotation cell sizes are 250 and 10 cubic meters. The optimization strategy is employed to determine the suitable number and size of flotation cells in each cleaning stage with the objective of maximizing NPV.

\subsubsection{Deterministic results}

Table 7-4 reports results of the deterministic problem when all financial and technical factors (i.e. price, feed grade, and kinetic coefficients) are considered as static values. The data indicate that the three-stage flotation circuit is capable of producing a final concentrate with $25 \%$ chalcopyrite at $97.34 \%$ global recovery value. The implementation of larger size cells in the rougher and scavenger stages and two $16-\mathrm{m}^{3}$ flotation cells in the cleaner stage leads to a total 25-year NPV of $\$ 1.67 \mathrm{E}+07$. The following sub-section summarizes the sensitivity of flotation circuit solution to the uncertainty of various techno-economic factors. 


\subsubsection{Sensitivity analysis}

\section{Copper price}

The original deterministic problem is separately solved at three levels of copper price (i.e. 4000, 6000, and 8000 (\$/ton) while other potentially uncertain constants (i.e. feed grade and kinetic coefficients) remain at their initial static values. Given the optimal size and number of flotation cells in each case, the associated financial and technical parameters to each design are determined at all three levels of copper price. As such, for each copper price case, three different flotation circuit designs are examined and compared against one another. Table 7-5 summarizes results of the sensitivity analysis study on the copper price.

As expected, the metal price element has a significant impact on the projected financial indicators. For instance, an increase in the copper price from $\$ 6000$ to $\$ 8000$ will improve the NPV by approximately 50\%. As shown in Table 7-5, the operating and capital cost of the flotation circuit remains unchanged unless the number and size of flotation cells are varied. Interestingly, these data indicate that different combinations of cell size and number may produce the similar NPV. In other words, price uncertainty does not necessarily affect the optimal design parameters as the optimal cell number and size at a specific price case will remain as one of the optimal solutions for other scenarios. This observation can be considered as a supplement to Cisternas et al. (2014)'s conclusion on the neglectable impact of metal price on the optimal superstructure design. Cisternas et al. (2014) examined the importance of metal price parameter on the optimal separation superstructure considering various objective functions, including NPV maximization, revenue maximization, and cost minimization. The results of this study indicated that the optimal superstructure remains unchanged at different levels of metal price when the NPV maximization is considered as the objective of the optimization problem. Therefore, it can be concluded that the uncertainty of metal price is an insignificant factor in the optimal design of cell-based flotation systems.

\section{Feed composition}

In the next phase of the study, the sensitivity of circuit solutions to the level of chalcopyrite concentration in the feed stream is evaluated. The level of metal grade in the 
plant feed can strongly affect the circuit separation performance as it may vary the associated kinetic coefficients. Despite this fact, in addition to the copper price, ore and gangue kinetic coefficients are kept constant in all three flotation banks. Like the prior phase of the study, the optimal circuit solution is determined for individual feed grade condition and then, the optimality of those data at other degrees of feed grade is investigated.

As indicated in Table 7-6, even the small variation in the feed composition, $0.5 \%$ increase, can substantially alter the value of circuit techno-economic measures. Solving the deterministic MINLP problem at distinct levels of feed grade results in obtaining a unique circuit design solution for each case. Nevertheless, the optimal circuit solution for one case has the capability to produce the maximum NPV at various degrees of feed grade, albeit at different levels of concentrate grade and yield. For example, the evaluation of the optimal circuit solution for the high-grade case (i.e. 5, 1, and 1 flotation cell with the volume of 205, 190 , and 50 cubic meters) at lower levels of feed grade shows that the maximum NPV is achievable at lower values of concentrate grade and yield. These observations indicate the importance of feed grade uncertainty in the optimal circuit design procedure when limitation on the final concentrate grade is specified. As such, unlike metal price, the uncertainty of feed grade parameter should be adequately considered in the stochastic circuit optimization problem.

\section{Kinetic coefficients}

The values of the kinetic coefficients in Table $7-4$ are varied by $\pm 20 \%$ and the deterministic circuit optimization problem is solved at the designated three levels of the kinetic coefficient for ore and gangue in all three flotation banks. Overall, results of the sensitivity analysis study on kinetic coefficients show a slight increase in the NPV at higher values of kinetic coefficients. The optimal circuit solutions show the selection of larger size cells for lower values of kinetic constants for producing the concentrate of $25 \%$ grade. The rise in the level of kinetic constants in these large flotation cells results in the lower concentrate grade and higher yield values. Altogether, these data indicate the significant impact of the kinetic coefficient factor on the optimal circuit solution. As a result, the stochastic optimization methodology must address the variability of this factor, besides feed grade, in the optimal design formulation. 
Table 7-5: Sensitivity analysis results on the metal price.

\begin{tabular}{|c|c|c|c|c|}
\hline & & Case 1: $\$ 4000$ & Case 2: $\$ 6000$ & Case 3: $\$ 8000$ \\
\hline \multirow{4}{*}{$\begin{array}{l}\text { Financial } \\
\text { outcomes }\end{array}$} & NPV $(\$)$ & $1.03 \mathrm{E}+07$ & $1.67 \mathrm{E}+07$ & $2.33 \mathrm{E}+07$ \\
\hline & $\operatorname{Rev}(\$)$ & $1.23 \mathrm{E}+08$ & $1.87 \mathrm{E}+08$ & $2.52 \mathrm{E}+08$ \\
\hline & Capex $(\$)$ & $1.73 \mathrm{E}+06$ & $1.73 \mathrm{E}+06$ & $1.73 \mathrm{E}+06$ \\
\hline & Opex $(\$)$ & $2.87 \mathrm{E}+06$ & $2.87 \mathrm{E}+06$ & $2.87 \mathrm{E}+06$ \\
\hline \multirow{3}{*}{$\begin{array}{l}\text { Optimal } \\
\text { circuit } \\
\text { solution at } \\
\$ 4000\end{array}$} & $N_{R}, V_{R}\left(m^{3}\right)$ & 4,197 & 4,197 & 4,197 \\
\hline & $N_{S}, V_{S}\left(m^{3}\right)$ & 1,213 & 1,213 & 1,213 \\
\hline & $N_{C}, V_{C}\left(m^{3}\right)$ & 2,16 & 2,16 & 2,16 \\
\hline \multirow{2}{*}{$\begin{array}{l}\text { Technical } \\
\text { outcomes }\end{array}$} & Concentrate grade (\%) & 25.02 & 25.02 & 25.02 \\
\hline & Yield $(\%)$ & 1.32 & 1.32 & 1.32 \\
\hline \multirow{4}{*}{$\begin{array}{l}\text { Financial } \\
\text { outcomes }\end{array}$} & $\operatorname{NPV}(\$)$ & $1.03 \mathrm{E}+07$ & $1.67 \mathrm{E}+07$ & $2.33 \mathrm{E}+07$ \\
\hline & $\operatorname{Rev}(\$)$ & $1.25 \mathrm{E}+08$ & $1.90 \mathrm{E}+08$ & $2.56 \mathrm{E}+08$ \\
\hline & Capex $(\$)$ & $1.99 \mathrm{E}+06$ & $1.99 \mathrm{E}+06$ & $1.99 \mathrm{E}+06$ \\
\hline & Opex $(\$)$ & $3.27 \mathrm{E}+06$ & $3.27 \mathrm{E}+06$ & $3.27 \mathrm{E}+06$ \\
\hline \multirow{3}{*}{$\begin{array}{c}\text { Optimal } \\
\text { circuit } \\
\text { solution at } \\
\$ 6000\end{array}$} & $N_{R}, V_{R}\left(m^{3}\right)$ & 4,194 & 4,194 & 4,194 \\
\hline & $N_{S}, V_{S}\left(m^{3}\right)$ & 2,182 & 2,182 & 2,182 \\
\hline & $N_{C}, V_{C}\left(m^{3}\right)$ & 2,16 & 2,16 & 2,16 \\
\hline \multirow{2}{*}{$\begin{array}{l}\text { Technical } \\
\text { outcomes }\end{array}$} & Concentrate grade (\%) & 25.15 & 25.15 & 25.15 \\
\hline & Yield (\%) & 1.34 & 1.34 & 1.34 \\
\hline \multirow{4}{*}{$\begin{array}{l}\text { Financial } \\
\text { outcomes }\end{array}$} & NPV (\$) & $1.03 \mathrm{E}+07$ & $1.67 \mathrm{E}+07$ & $2.33 \mathrm{E}+07$ \\
\hline & $\operatorname{Rev}(\$)$ & $1.25 \mathrm{E}+08$ & $1.91 \mathrm{E}+08$ & $2.56 \mathrm{E}+08$ \\
\hline & Capex $(\$)$ & $2.04 \mathrm{E}+06$ & $2.04 \mathrm{E}+06$ & $2.04 \mathrm{E}+06$ \\
\hline & Opex $(\$)$ & $3.52 \mathrm{E}+06$ & $3.52 \mathrm{E}+06$ & $3.52 \mathrm{E}+06$ \\
\hline \multirow{3}{*}{$\begin{array}{c}\text { Optimal } \\
\text { circuit } \\
\text { solution at } \\
\$ 8000\end{array}$} & $N_{R}, V_{R}\left(m^{3}\right)$ & 4,211 & 4,211 & 4,211 \\
\hline & $N_{S}, V_{S}\left(m^{3}\right)$ & 2,196 & 2,196 & 2,196 \\
\hline & $N_{C}, V_{C}\left(m^{3}\right)$ & 1,33 & 1,33 & 1,33 \\
\hline \multirow{2}{*}{$\begin{array}{l}\text { Technical } \\
\text { outcomes }\end{array}$} & Concentrate grade (\%) & 25.07 & 25.07 & 25.07 \\
\hline & Yield $(\%)$ & 1.35 & 1.35 & 1.35 \\
\hline
\end{tabular}


Table 7-6: Sensitivity analysis results on the feed grade.

\begin{tabular}{|c|c|c|c|c|}
\hline & & $\mathrm{G}_{\mathrm{F}} 1: 0.5 \%$ & $\mathrm{G}_{\mathrm{F}} 2: 1.0 \%$ & $\mathrm{G}_{\mathrm{F}} 3: 1.5 \%$ \\
\hline \multirow{4}{*}{ Financial outcomes } & NPV (\$) & $7.17 \mathrm{E}+06$ & $1.67 \mathrm{E}+07$ & $2.51 \mathrm{E}+07$ \\
\hline & $\operatorname{Rev}(\$)$ & $9.13 \mathrm{E}+07$ & $1.82 \mathrm{E}+08$ & $2.71 \mathrm{E}+08$ \\
\hline & Capex $(\$)$ & $1.70 \mathrm{E}+06$ & $1.70 \mathrm{E}+06$ & $1.70 \mathrm{E}+06$ \\
\hline & Opex $(\$)$ & $2.62 \mathrm{E}+06$ & $2.62 \mathrm{E}+06$ & $2.62 \mathrm{E}+06$ \\
\hline \multirow{3}{*}{$\begin{array}{l}\text { Optimal } \\
\text { circuit solution } \\
\text { at } \mathrm{G}_{\mathrm{F}} \text { of } 0.5 \%\end{array}$} & $N_{R}, V_{R}\left(m^{3}\right)$ & 4,130 & 4,130 & 4,130 \\
\hline & $N_{S}, V_{S}\left(m^{3}\right)$ & 2,200 & 2,200 & 2,200 \\
\hline & $N_{C}, V_{C}\left(m^{3}\right)$ & 1,15 & 1,15 & 1,15 \\
\hline \multirow{2}{*}{ Technical outcomes } & Concentrate grade (\%) & 25.24 & 29.73 & 31.55 \\
\hline & Yield (\%) & 0.64 & 1.08 & 1.51 \\
\hline \multirow{4}{*}{ Financial outcomes } & NPV (\$) & $7.20 \mathrm{E}+06$ & $1.67 \mathrm{E}+07$ & $2.62 \mathrm{E}+07$ \\
\hline & $\operatorname{Rev}(\$)$ & $9.52 \mathrm{E}+07$ & $1.90 \mathrm{E}+08$ & $2.85 \mathrm{E}+08$ \\
\hline & Capex $(\$)$ & $1.99 \mathrm{E}+06$ & $1.99 \mathrm{E}+06$ & $1.99 \mathrm{E}+06$ \\
\hline & Opex $(\$)$ & $3.27 \mathrm{E}+06$ & $3.27 \mathrm{E}+06$ & $3.27 \mathrm{E}+06$ \\
\hline \multirow{3}{*}{$\begin{array}{l}\text { Optimal } \\
\text { circuit solution } \\
\text { at } \mathrm{G}_{\mathrm{F}} \text { of } 1.0 \%\end{array}$} & $N_{R}, V_{R}\left(m^{3}\right)$ & 4,194 & 4,194 & 4,194 \\
\hline & $N_{S}, V_{S}\left(m^{3}\right)$ & 2,182 & 2,182 & 2,182 \\
\hline & $N_{C}, V_{C}\left(m^{3}\right)$ & 2,16 & 2,16 & 2,16 \\
\hline \multirow{2}{*}{ Technical outcomes } & Concentrate grade (\%) & 19.19 & 25.15 & 28.04 \\
\hline & Yield (\%) & 0.88 & 1.34 & 1.79 \\
\hline \multirow{4}{*}{ Financial outcomes } & NPV (\$) & $7.17 \mathrm{E}+06$ & $1.67 \mathrm{E}+07$ & $2.63 \mathrm{E}+07$ \\
\hline & $\operatorname{Rev}(\$)$ & $9.57 \mathrm{E}+07$ & $1.91 \mathrm{E}+08$ & $2.87 \mathrm{E}+08$ \\
\hline & Capex $(\$)$ & $2.05 \mathrm{E}+06$ & $2.05 \mathrm{E}+06$ & $2.05 \mathrm{E}+06$ \\
\hline & Opex $(\$)$ & $3.51 \mathrm{E}+06$ & $3.51 \mathrm{E}+06$ & $3.51 \mathrm{E}+06$ \\
\hline \multirow{3}{*}{$\begin{array}{c}\text { Optimal } \\
\text { circuit solution } \\
\text { at } \mathrm{G}_{\mathrm{F}} \text { of } 1.5 \%\end{array}$} & $N_{R}, V_{R}\left(m^{3}\right)$ & 5,205 & 5,205 & 5,205 \\
\hline & $N_{S}, V_{S}\left(m^{3}\right)$ & 1,190 & 1,190 & 1,190 \\
\hline & $N_{C}, V_{C}\left(m^{3}\right)$ & 1,50 & 1,50 & 1,50 \\
\hline \multirow{2}{*}{ Technical outcomes } & Concentrate grade (\%) & 15.43 & 21.79 & 25.22 \\
\hline & Yield (\%) & 1.10 & 1.56 & 2.00 \\
\hline
\end{tabular}


Table 7-7: Sensitivity analysis results on the kinetic coefficient.

\begin{tabular}{|c|c|c|c|c|}
\hline & & Low $\mathrm{K}$ & Medium K & High K \\
\hline \multirow{4}{*}{ Financial outcomes } & NPV (\$) & $1.62 \mathrm{E}+07$ & $1.67 \mathrm{E}+07$ & $1.70 \mathrm{E}+07$ \\
\hline & $\operatorname{Rev}(\$)$ & $1.85 \mathrm{E}+08$ & $1.90 \mathrm{E}+08$ & $1.92 \mathrm{E}+08$ \\
\hline & Capex $(\$)$ & $1.93 \mathrm{E}+06$ & $1.93 \mathrm{E}+06$ & $1.93 \mathrm{E}+06$ \\
\hline & Opex (\$) & $3.57 \mathrm{E}+06$ & $3.57 \mathrm{E}+06$ & $3.57 \mathrm{E}+06$ \\
\hline \multirow{3}{*}{$\begin{array}{l}\text { Optimal } \\
\text { circuit solution at low } \mathrm{K}\end{array}$} & $N_{R}, V_{R}\left(m^{3}\right)$ & 3,250 & 3,250 & 3,250 \\
\hline & $N_{S}, V_{S}\left(m^{3}\right)$ & 2,250 & 2,250 & 2,250 \\
\hline & $N_{C}, V_{C}\left(m^{3}\right)$ & 1,45 & 1,45 & 1,45 \\
\hline \multirow{2}{*}{ Technical outcomes } & Concentrate grade (\%) & 25.04 & 22.85 & 20.93 \\
\hline & Yield (\%) & 1.31 & 1.47 & 1.63 \\
\hline \multirow{4}{*}{ Financial outcomes } & NPV (\$) & $1.61 \mathrm{E}+07$ & $1.67 \mathrm{E}+07$ & $1.67 \mathrm{E}+07$ \\
\hline & $\operatorname{Rev}(\$)$ & $1.85 \mathrm{E}+08$ & $1.90 \mathrm{E}+08$ & $1.93 \mathrm{E}+08$ \\
\hline & Capex $(\$)$ & $1.99 \mathrm{E}+06$ & $1.99 \mathrm{E}+06$ & $1.99 \mathrm{E}+06$ \\
\hline & Opex $(\$)$ & $3.27 \mathrm{E}+06$ & $3.27 \mathrm{E}+06$ & $3.27 \mathrm{E}+06$ \\
\hline \multirow{3}{*}{$\begin{array}{l}\text { Optimal } \\
\text { circuit solution at medium } \\
\mathrm{K}\end{array}$} & $N_{R}, V_{R}\left(m^{3}\right)$ & 4,194 & 4,194 & 4,194 \\
\hline & $N_{S}, V_{S}\left(m^{3}\right)$ & 2,182 & 2,182 & 2,182 \\
\hline & $N_{C}, V_{C}\left(m^{3}\right)$ & 2,16 & 2,16 & 2,16 \\
\hline \multirow{2}{*}{ Technical outcomes } & Concentrate grade (\%) & 27.06 & 25.15 & 23.43 \\
\hline & Yield (\%) & 1.21 & 1.34 & 1.46 \\
\hline \multirow{4}{*}{ Financial outcomes } & NPV (\$) & $1.55 \mathrm{E}+07$ & $1.65 \mathrm{E}+07$ & $1.69 \mathrm{E}+07$ \\
\hline & $\operatorname{Rev}(\$)$ & $1.76 \mathrm{E}+08$ & $1.86 \mathrm{E}+08$ & $1.91 \mathrm{E}+08$ \\
\hline & Capex $(\$)$ & $1.88 \mathrm{E}+06$ & $1.88 \mathrm{E}+06$ & $1.88 \mathrm{E}+06$ \\
\hline & Opex $(\$)$ & $2.58 \mathrm{E}+06$ & $2.58 \mathrm{E}+06$ & $2.58 \mathrm{E}+06$ \\
\hline \multirow{3}{*}{$\begin{array}{l}\text { Optimal } \\
\text { circuit solution at high } \mathrm{K}\end{array}$} & $N_{R}, V_{R}\left(m^{3}\right)$ & 4,140 & 4,140 & 4,140 \\
\hline & $N_{S}, V_{S}\left(m^{3}\right)$ & 4,80 & 4,80 & 4,80 \\
\hline & $N_{C}, V_{C}\left(m^{3}\right)$ & 1,27 & 1,27 & 1,27 \\
\hline \multirow{2}{*}{ Technical outcomes } & Concentrate grade (\%) & 28.19 & 26.59 & 25.04 \\
\hline & Yield (\%) & 1.11 & 1.24 & 1.35 \\
\hline
\end{tabular}


Table 7-8: Statistical measures of the input parameters in the stochastic circuit design problem.

\begin{tabular}{|c|c|c|}
\hline Input Factors & Mean & Standard Deviation \\
\hline$q(\$)$ & 6000 & --- \\
\hline$T_{O, F}($ ton $/ \mathrm{hr})$ & 10 & 3 \\
\hline$T_{G, F}($ ton $/ \mathrm{hr})$ & 990 & --- \\
\hline$K_{O, R}(1 / \mathrm{min})$ & 0.2250 & 0.0900 \\
\hline$K_{O, S}(1 / \mathrm{min})$ & 0.2000 & 0.0800 \\
\hline$K_{O, C}(1 / \mathrm{min})$ & 0.2750 & 0.1100 \\
\hline$K_{G, R}(1 / \mathrm{min})$ & 0.0050 & 0.0020 \\
\hline$K_{G, S}(1 / \mathrm{min})$ & 0.0100 & 0.0040 \\
\hline$K_{G, C}(1 / \mathrm{min})$ & 0.0075 & 0.0030 \\
\hline
\end{tabular}

\subsubsection{Stochastic circuit design optimization}

Given the results of the sensitivity analysis study, the SAA approach and Taguchi's methodology are utilized to find the optimal number and size of flotation cells for the threestage flotation system. The resultant stochastic approach considers the feed grade and kinetic coefficients as uncertain parameters while the metal price value is kept constant at $\$ 6000$. As such, chalcopyrite flow rate in feed stream (feed grade) and kinetic coefficient parameters are incorporated in the original model with uniform, normal, and lognormal probability distribution functions. For each case, 1000 random numbers are generated, the simulation is repeated 20 times, and then alternative optimal solutions are selected. For each statistical distribution, two circuit solutions are chosen according to their profitability and frequency among all 20 results. Afterwards, Taguchi's method is applied to the original MINLP model using three carefully selected numbers representing feed grade and kinetic coefficients' uncertainties (the detailed description of Taguchi's method was provided in previous chapter). Finally, these seven stochastic circuit solutions are compared against the optimal design obtained from the deterministic formulation and optimal flotation design alternatives are suggested. Table 7-8 shows the statistical measures of uncertain input parameters used in the stochastic optimization procedure. 

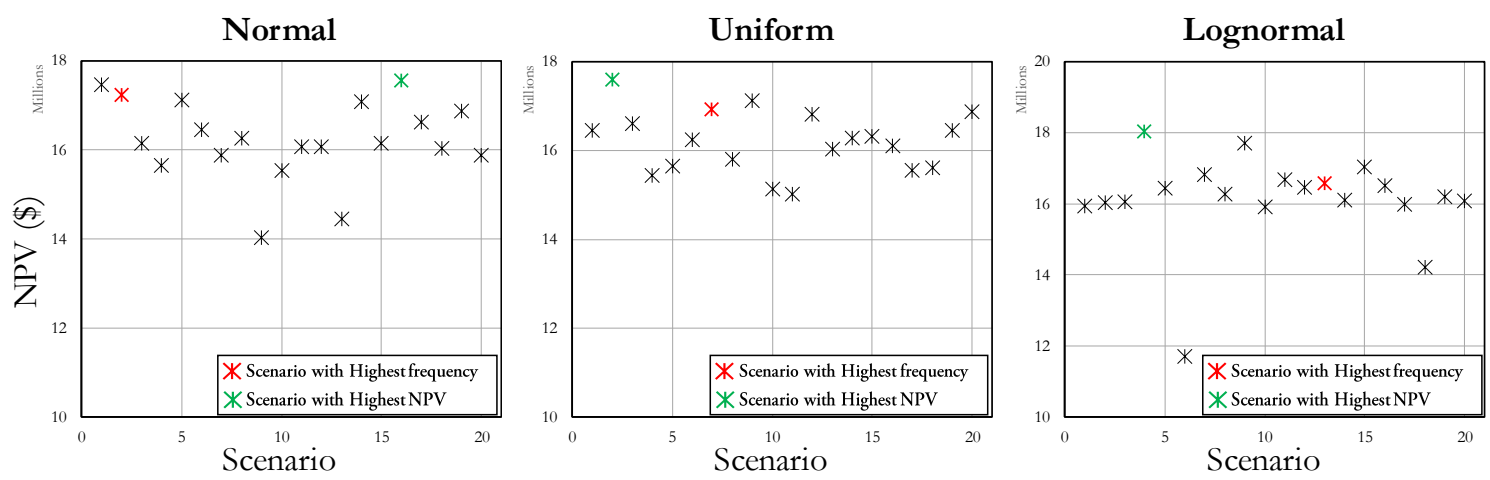

Figure 7-3: The value of objective function for different scenarios of the stochastic optimization.

Figure 7-3 depicts the value of objective functions (NPV) for all three cases of statistical distributions throughout 20 scenarios of stochastic optimization. Of all scenarios, one solution with the highest level of NPV (green marker) and one with the highest frequency (red marker) are selected as the alternative optimal circuit solutions. Additionally, Figure 74 indicates the results of SAA approach and Taguchi's method alongside the deterministic circuit solution.

The comparison between stochastic and deterministic circuit design solutions (Figure 74) clearly demonstrate the significance of considering uncertainty in the flotation circuit design procedure. Furthermore, these data show that input parameters' statistical distribution can play a significant role, as each distribution type may produce a different circuit solution. Interestingly, the results of all 20 scenarios in the SAA approach, regardless of distribution shape, select a cleaner bank containing only one flotation cell with the size of between 36 to $40-\mathrm{m}^{3}$. However, Taguchi's method result confirms the solution of the deterministic solution with two small flotations cells in the cleaner stage. More interestingly, according to the 20 scenarios observations, the highest frequency solution (and possibly the most robust solution) is identical in all cases of statistical distribution. 


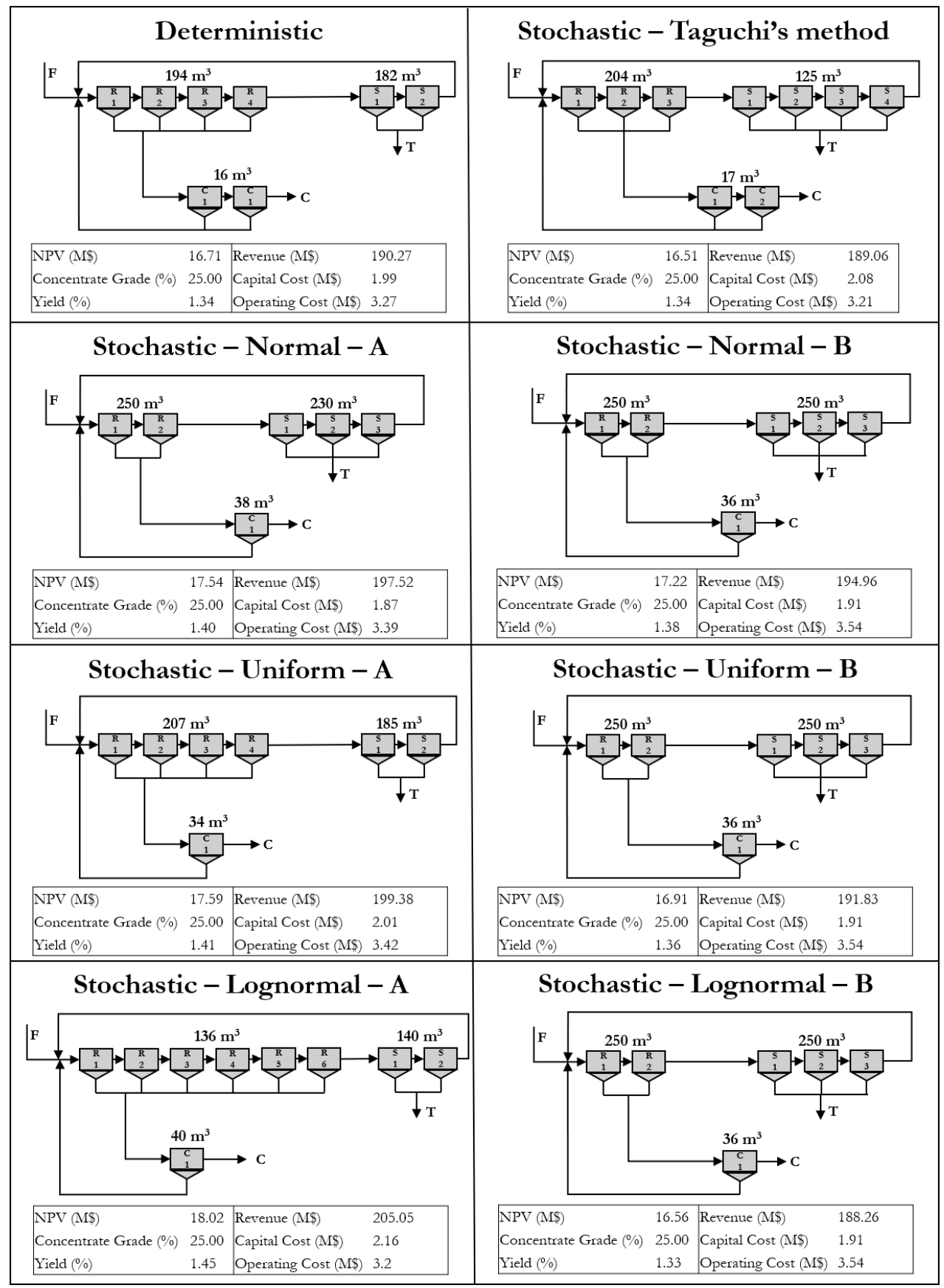

Figure 7-4: Comparison between the optimal results of the determinsitc, Taguchi, and Sample Averege Approximation methodologies. [Optimal scenarios with highest NPV (A) and optimal scenarios with highest frequency (B)] 
Following the result of this phase of the study, the optimality of stochastic circuit solutions in the original deterministic model is investigated. The results of this analysis indicate that none of the stochastic circuit solutions are placed in the category of optimal solutions for the deterministic problem. These observations verify the essential need for considering uncertainty through a stochastic flotation circuit analysis. Altogether, six optimal circuit solution candidates are selected for further analysis and possible laboratory- and pilotscale experimentations.

\subsection{Summary and conclusions}

The optimal selection of the size and number of cells in the cell-based flotation circuit design is a vital part of an effective flotation design procedure as these factors directly define the circuit financial measures. Additionally, a comprehensive circuit optimization strategy must address the uncertainty associated with various techno-economic parameters, including price, feed grade, and kinetic coefficients. In this study, an innovative stochastic optimization approach was developed for finding the optimal circuit solution with the objective of maximizing NPV. After completion of a systematic sensitivity analysis study, feed grade and kinetic coefficient were incorporated in the optimization model based on various statistical distributions. Sample Average Approximation approach, as a Monte Carlo-based technique, and Taguchi's method were employed to select alternative optimal flotation circuit solutions. The following conclusions are drawn from this chapter:

- Results of the proposed circuit optimization strategy indicate that multiple circuit solutions (number and size of flotation cells) may produce a maximum NPV.

- Despite the importance of metal price value in determining the circuit profitability, its associated variability does not necessarily affect the optimal circuit solution.

- Feed grade and kinetic coefficient uncertainties have significant role in defining the metallurgical performance of the flotation circuit, thereby affecting the circuit profitability. 
- The results of the stochastic optimization approach demonstrated the importance of statistical distribution type, by which uncertain factors were incorporated in the original model. Therefore, comprehensive experiments are required to properly specify the distribution of uncertain input parameters.

- The resultant circuit solutions from the stochastic optimization methodology do not necessarily maximize the objective function in the deterministic model. This important observation demonstrates the importance of considering uncertainty in the mineral processing circuit design procedure.

\subsection{Bibliography}

Abu-Ali M, Sabour SA. Optimizing the design of flotation circuits: an economic approach. Minerals engineering. Elsevier; 2003;16(1):55-8.

Amini SH, Noble A. Application of linear circuit analysis in evaluation of mineral processing circuit design under uncertainty. Minerals Engineering. Elsevier; 2017;102:18-29.

Bardossy MG, Raghavan S. An inexact sample average approximation approach for the stochastic connected facility location problem. Networks. Wiley Online Library; 2017;

Cisternas LA, Gálvez ED, Méndez DA. Optimal design of flotation circuits including selection of bank cells, column cells and regrinding mills. 2nd Mercosur Congress on Chemical Engineering, 4th Mercosur Congress on Process Systems Engineering, Rio de Janeiro. 2005.

Cisternas LA, Lucay F, Gálvez ED. Effect of the objective function in the design of concentration plants. Minerals Engineering. Elsevier; 2014;63:16-24.

Cisternas LA, Méndez DA, Gálvez ED, Jorquera RE. A MILP model for design of flotation circuits with bank/column and regrind/no regrind selection. International Journal of Mineral Processing. Elsevier; 2006;79(4):253-63. 
Fuerstenau MC, Han KN. Principles of mineral processing. SME; 2003.

Fuerstenau MC, Jameson GJ, Yoon R-H. Froth flotation: a century of innovation. SME; 2007.

Ghobadi P, Yahyaei M, Banisi S. Optimization of the performance of flotation circuits using a genetic algorithm oriented by process-based rules. International Journal of Mineral Processing. Elsevier; 2011;98(3):174-81.

Green J. The optimisation of flotation networks. International journal of mineral processing. Elsevier; 1984;13(2):83-103.

Guria C, Varma M, Mehrotra SP, Gupta SK. Simultaneous optimization of the performance of flotation circuits and their simplification using the jumping gene adaptations of genetic algorithm-II: More complex problems. International Journal of Mineral Processing. Elsevier; 2006;79(3):149-66.

Guria C, Verma M, Gupta SK, Mehrotra SP. Simultaneous optimization of the performance of flotation circuits and their simplification using the jumping gene adaptations of genetic algorithm. International Journal of Mineral Processing. Elsevier; 2005 a;77(3):165-85.

Guria C, Verma M, Mehrotra SP, Gupta SK. Multi-objective optimal synthesis and design of froth flotation circuits for mineral processing, using the jumping gene adaptation of genetic algorithm. Industrial \& engineering chemistry research. ACS Publications; 2005 b;44(8):2621-33.

Jamett N, Cisternas LA, Vielma JP. Solution strategies to the stochastic design of mineral flotation plants. Chemical Engineering Science. Elsevier; 2015;134:850-60.

Kleywegt AJ, Shapiro A, Homem-de-Mello T. The sample average approximation method for stochastic discrete optimization. SIAM Journal on Optimization. SIAM; 2002;12(2):479-502.

Mak W-K, Morton DP, Wood RK. Monte Carlo bounding techniques for determining solution quality in stochastic programs. Operations research letters. Elsevier; 1999;24(1):47-56. 
Mular A. The estimation of preliminary capital costs. Mineral Processing Plant Design, New York, New York, USA: Society of Mining Engineers of the AIME Inc. 1978;

Noble A, Luttrell GH. The matrix reduction algorithm for solving separation circuits. Minerals Engineering. Elsevier; 2014;64:97-108.

Reuter M, Van Deventer J. The use of linear programming in the optimal design of flotation circuits incorporating regrind mills. International Journal of Mineral Processing. Elsevier; 1990;28(1-2):15-43.

Reuter M, Van Deventer J, Green J, Sinclair M. Optimal design of mineral separation circuits by use of linear programming. Chemical Engineering Science. Elsevier; 1988;43(5):1039-49.

Schena G, Villeneuve J, Noël Y. A method for a financially efficient design of cell-based flotation circuits. International journal of mineral processing. Elsevier; 1996;46(1):1-20.

Schena G, Zanin M, Chiarandini A. Procedures for the automatic design of flotation networks. International journal of mineral processing. Elsevier; 1997;52(2):137-60.

Tawarmalani M, Sahinidis NV. A polyhedral branch-and-cut approach to global optimization. Mathematical Programming. Springer; 2005;103(2):225-49.

Verweij B, Ahmed S, Kleywegt AJ, Nemhauser G, Shapiro A. The sample average approximation method applied to stochastic routing problems: a computational study. Computational Optimization and Applications. Springer; 2003;24(2):289-333.

Wills BA. Wills' mineral processing technology: an introduction to the practical aspects of ore treatment and mineral recovery. Butterworth-Heinemann; 2011.

Yingling JC. Parameter and configuration optimization of flotation circuits, part I. A review of prior work. International journal of mineral processing. Elsevier; 1993;38(1):2140. 


\section{Chapter 8}

\section{Conclusions and Recommendations}

Mineral processing is the science of physically separating valuable materials within the run-of-mine with the objective of producing the high-grade concentrate for downstream users. Often, single unit separation technologies are incapable of producing products which meet the required quality and quantity specifications. Therefore, separation circuit designers employ multi-stage separation circuit configurations to produce a sufficient product based on the grade constraints.

Over the last 40 years, many studies addressed the optimal design of separation circuit configuration. Unfortunately, most of these available circuit design methodologies do not consider the stochasticity of influential techno-economic factors in the circuit design procedure. Nevertheless, numerous studies and observations confirmed the inherent uncertainty associated with the design parameters, including feed grade, operational parameters, and product price. Neglecting the significance of parameters' uncertainties in the circuit design procedure may result in failing to achieve the optimal or even sub-optimal separation circuit solutions.

This work has provided various circuit design and optimization methodologies for the optimal separation circuit design under uncertainty. First, an analytical circuit uncertainty quantification approach was developed based on the principles of linear circuit analysis and law of propagation of error. This novel technique can help circuit designers to quantify the separation circuit uncertainty during the early design stages with the limited input data. 
Second, the analytical approach was joined with the principles of functional unit concept to evaluate the level of separation circuit uncertainty. Several generic circuit design rules were then proposed to effectively explain the fundamental trends and uncertainty expectations during the circuit design procedure.

Third, the similar approach was utilized as a global sensitivity analysis tool to accurately determine the influential factors on the circuit uncertainty propagation without need to the sophisticated mathematical models and software packages. The identification of these important parameters can help circuit designers to effectively control the sources of performance variance, thereby improving the robustness of the final separation circuit design.

Fourth, the potential capabilities of these analytical methodologies were investigated in an extensive separation experiments. The performance variability of several separation circuit configurations was evaluated using an electrostatic separator. The final outcomes of this systematic experimental program were compared with the theoretical conclusions derived form the circuit uncertainty quantification methodologies. In general, these validation experiments confirm the exceptional capacity of the proposed approach in analyzing and evaluating circuit designs under uncertainty.

Fifth, a novel implementation of Taguchi's method, as an experimental design technique, was provided to determine the level of uncertainty associated with various circuit performance indicators alongside their resultant statistical distributions. This methodology can be employed in the circuit analysis procedure as an alternative to the traditional Monte Carlo simulation.

Sixth, an innovative techno-economic strategy was developed to find the optimal cellbased flotation circuit design parameters for the cases of uncertainty in the feed grade and kinetic coefficient parameters. Sample average approximation methodology was combined with the original deterministic approach to effectively incorporate internal and external sources of uncertainty in the optimal circuit design procedure. The resultant strategy was utilized to determine the size and number of flotation cells in each cleaning stage with the objective of maximizing net present value. 
Collectively, the proposed tools may be utilized in the circuit design procedure to effectively analyze, evaluate, and optimize mineral processing circuits under uncertainty. During the preliminary greenfield design stages, when extensive data sets are costly and largely non-existent, the proposed methodologies can be employed to provide several optimal or sub-optimal circuit configurations. These alternative circuit solutions may be then evaluated via further experimental and simulation works.

To the author's knowledge, following original contributions have been presented in this work:

1. An analytical circuit uncertainty quantification tool. This technique accurately estimates the circuit uncertainty propagation for different separation performance indicators. This method only requires the separation unit arrangements, stream interconnections diagram, and estimates of the mean and uncertainty of input parameters, including stage recovery and feed grade, to provide these fundamental insights.

2. The generic design rules for predicting the direction of uncertainty propagation in modified circuit applications. These simple heuristics not only supplement more complex optimization protocols but provide insight on why certain circuit configurations propagate uncertainty at distinct levels.

3. A global sensitivity analysis tool for identifying the influential factors on the circuit uncertainty propagation. Though many researchers utilize various sensitivity analysis algorithms with excellent capabilities for determining influential parameters in the circuit performance, their complex mathematical basis necessitates the use of specialized software packages. The novel proposed technique, with no need for exhaustive datasets, provides highly beneficial information to circuit designers working on preliminary greenfield or operating design problems.

4. The empirical evaluation of circuit designs under uncertainty using dry-based separation experiments. Despite recent scientific developments, none of the studies include a comprehensive empirical validation of techniques for determination of the circuit uncertainty propagation. This research has included systematic experimental tests which analyzed circuit performance and uncertainty while validating much of the proposed methodology. 
5. An alternative circuit uncertainty quantification technique by the means of experimental design tool. Despite the popularity of Monte Carlo simulation for solving stochastic problems, the substantial number of simulation iterations limits the applicability of this tool in the available separation circuit design software packages. Taguchi's method, as an effective alternative technique, can be utilized to properly predict circuit uncertainty propagation during the early design stages.

6. A novel techno-economic strategy for the stochastic optimization of cell-based flotation circuits. Given the importance of flotation cell specifications in determining the flotation metallurgical and financial measures, a comprehensive optimization methodology was proposed to find the most profitable circuit design while considering cell number and size as decision variables. The proposed methodology incorporates the stochasticity of various technical and economic factors in the optimal design procedure. The author's algorithm provides series of optimal circuit design alternatives.

Following papers were published from the results of the current dissertation:

Amini SH, Noble A. Application of linear circuit analysis in evaluation of mineral processing circuit design under uncertainty. Minerals Engineering. Elsevier; 2017;102:18-29.

Amini SH, Noble A. Application of Taguchi's method to uncertainty assessment and sensitivity analysis for mineral processing circuits; 2017 SME Annual Meeting \& Exhibit. Denver, CO. Preprint No 17-011.

Finally, the author of this dissertation recommends the following items for continued study:

1. Development of a comprehensive optimal circuit design protocol considering both circuit selectivity and uncertainty factors. Currently, the separation circuit design methodologies seek to find the optimal or sub-optimal circuit solution according to the circuit selectivity or uncertainty, not both, measures. However, an effective circuit design procedure must adequately evaluate alternative circuit configurations and rank them according to both factors. 
2. Further validation of linear circuit analysis approach in real world processing systems. The current study has validated the capability of linear circuit analysis and functional unit concept in determining the level of circuit uncertainty quantification. However, more systematic physical experiments can be carried out to investigate all potential capacities of this valuable technique in determining circuit selectivity outcomes (e.g. recovery, grade, separation efficiency, moment of inertia, and yield score) and in identifying the influential factors.

3. Development of a profit-risk-based optimization model for an effective selection of the optimal flotation circuits. Mineral processing is a costly portion of the raw material production chain. Given this fact and the high level of risk associated with this system, a comprehensive optimization model should find the optimal circuit solution that maximizes the projected profit while minimizing the risk factor. However, due to the complexity of circuit design optimization models, development of such a model is a very challenging engineering task. 